\title{
THE RADIAL VELOCITY EXPERIMENT (RAVE): FOURTH DATA RELEASE
}

\author{
G. Kordopatis ${ }^{1}$, G. Gilmore ${ }^{1}$, M. Steinmetz ${ }^{2}$, C. Boeche ${ }^{3}$, G. M. Seabroke ${ }^{4}$, A. Siebert ${ }^{5}$, T. Zwitter ${ }^{6,7}$, \\ J. Binney ${ }^{8}$, P. De Laverny ${ }^{9}$, A. Recio-Blanco ${ }^{9}$, M. E. K. Williams ${ }^{2}$, T. Piffl $^{2}$, H. EnKe ${ }^{2}$, S. Roeser ${ }^{3}$, A. Bijaoui ${ }^{9}$, \\ R. F. G. Wyse ${ }^{10}$, K. Freeman $^{11}$, U. Munari ${ }^{12}$, I. Carrillo ${ }^{2}$, B. Anguiano ${ }^{13,14}$, D. Burton ${ }^{11,15}$, R. Campbell ${ }^{16}$, \\ C. J. P. Cass ${ }^{15}$, K. Fiegert ${ }^{15}$, M. Hartley ${ }^{15}$, Q. A. Parker ${ }^{13,14,17}$, W. Reid ${ }^{14,17}$, A. RitTer ${ }^{18}$, K. S. Russell ${ }^{15}$, \\ M. Stupar ${ }^{15}$, F. G. Watson ${ }^{15}$, O. Bienaymé ${ }^{5}$, J. Bland-Hawthorn ${ }^{19}$, O. Gerhard ${ }^{20}$, B. K. Gibson ${ }^{21}$, E. K. Grebel ${ }^{3}$,

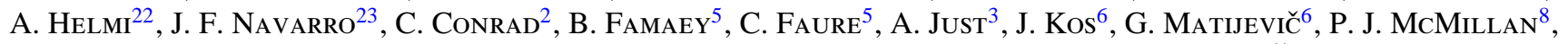 \\ I. Minchev ${ }^{2}$, R. Scholz ${ }^{2}$, S. Sharma ${ }^{19}$, A. Siviero ${ }^{2}$, E. Wylie de Boer ${ }^{11}$, AND M. ŽerJaL ${ }^{6}$ \\ ${ }^{1}$ Institute of Astronomy, University of Cambridge, Madingley Road, Cambridge, CB3 0HA, UK; gkordo@ ast.cam.ac.uk \\ ${ }^{2}$ Leibniz-Institut für Astrophysik Potsdam, An der Sternwarte 16, D-14482 Potsdam, Germany \\ ${ }^{3}$ Astronomisches Rechen-Institut, Zentrum für Astronomie der Universität Heidelberg, Mönchhofstr. 12-14, D-69120 Heidelberg, Germany \\ ${ }^{4}$ Mullard Space Science Laboratory, University College London, Holmbury St. Mary, Dorking, Surrey, RH5 6NT, UK \\ ${ }^{5}$ Observatoire Astronomique de Strasbourg, Université de Strasbourg, CNRS, UMR 7550, 11 rue de l'Université, F-67000 Strasbourg, France \\ ${ }^{6}$ Faculty of Mathematics and Physics, University of Ljubljana, Jadranska 19, 1000 Ljubljana, Slovenia \\ ${ }^{7}$ Center of Excellence SPACE-SI, Askerceva cesta 12, 1000 Ljubljana, Slovenia \\ ${ }^{8}$ Rudolf Peierls Centre for Theoretical Physics, Keble Road, Oxford, OX1 3NP, UK \\ ${ }^{9}$ Laboratoire Lagrange, UMR 7293, Université de Nice Sophia Antipolis, CNRS, Observatoire de la Côte d'Azur, BP4229, F-06304 Nice, France \\ 10 Johns Hopkins University, 3400 North Charles Street, Baltimore, MD 21218, USA \\ ${ }^{11}$ Research School of Astronomy and Astrophysics, Australian National University, Cotter Road, Weston, ACT 2611, Australia \\ ${ }^{12}$ INAF National Institute of Astrophysics, Astronomical Institute of Padova, I-36012 Asiago (VI), Italy \\ ${ }_{13}$ Australian Astronomical Observatory, P.O. Box 915, North Ryde, NSW 1670, Australia \\ ${ }^{14}$ Department of Physics and Astronomy, Macquarie University, Sydney, NSW 2109, Australia \\ ${ }_{15}$ Anglo-Australian Observatory, P.O. Box 296, Epping, NSW 1710, Australia \\ ${ }^{16}$ Western Kentucky University, Bowling Green, KY 42101, USA \\ ${ }_{17}$ Macquarie Research Centre for Astronomy, Astrophysics and Astrophotonics, Sydney, NSW 2109, Australia \\ ${ }^{18}$ National Central University, 300 Zhongda Road, Zhongli City, Taoyuan County 325, Taiwan, Republic of China \\ ${ }^{19}$ Sydney Institute for Astronomy, School of Physics A28, University of Sydney, NSW 2006, Australia \\ ${ }^{20}$ Max-Planck-Institut fuer Ex. Physik, Giessenbachstrasse, D-85748 Garching b. Muenchen, Germany \\ ${ }^{21}$ Jeremiah Horrocks Institute, University of Central Lancashire, Preston, PR1 2HE, UK \\ 22 Kapteyn Astronomical Institute, University of Groningen, P.O. Box 800, NL-9700 AV Groningen, The Netherlands \\ ${ }^{23}$ Department of Physics and Astronomy, University of Victoria, Victoria, BC, Canada \\ Received 2013 July 11; accepted 2013 September 2; published 2013 October 17
}

\begin{abstract}
We present the stellar atmospheric parameters (effective temperature, surface gravity, overall metallicity), radial velocities, individual abundances, and distances determined for 425,561 stars, which constitute the fourth public data release of the RAdial Velocity Experiment (RAVE). The stellar atmospheric parameters are computed using a new pipeline, based on the algorithms of MATISSE and DEGAS. The spectral degeneracies and the Two Micron All Sky Survey photometric information are now better taken into consideration, improving the parameter determination compared to the previous RAVE data releases. The individual abundances for six elements (magnesium, aluminum, silicon, titanium, iron, and nickel) are also given, based on a special-purpose pipeline that is also improved compared to that available for the RAVE DR3 and Chemical DR1 data releases. Together with photometric information and proper motions, these data can be retrieved from the RAVE collaboration Web site and the Vizier database.
\end{abstract}

Key words: catalogs - stars: abundances - stars: fundamental parameters - surveys - techniques: spectroscopic

Online-only material: color figures

\section{INTRODUCTION}

The assembly history of the Milky Way can be obtained by analyzing the positions, kinematics, ages, and chemical compositions of large statistical samples of Galactic stars (Freeman $\&$ Bland-Hawthorn 2002). In addition to the identification and characterization of hierarchical signatures (e.g., Helmi et al. 1999; Abadi et al. 2003; Sales et al. 2009), the precise measurement of the age-metallicity relation in the solar neighborhood, for a very large sample of stars, allows us to establish, among much else, the strength and the importance of radial migration in the Galaxy, perhaps a key ingredient for Galactic evolution (Sellwood \& Binney 2002; van der Kruit \& Freeman 2011).

Ideally, one would need stellar spectra and precise distances to achieve such a goal. Nevertheless, even in the case where par- allaxes are not available, it is still possible to estimate statistically valuable ages and distances of the stars by measuring from their spectra their atmospheric parameters (effective temperature, $T_{\text {eff }}$, surface gravity, $\log g$, overall metallicity, $[\mathrm{M} / \mathrm{H}]^{24}$ ) and projecting them afterward on theoretical stellar isochrones (Breddels et al. 2010; Zwitter et al. 2010; Burnett et al. 2011; Kordopatis et al. 2011b; Binney et al. 2013).

In the past decade, the advent of multi-fiber spectrographs, combined with large telescopes, has allowed the astronomical community to obtain such very large amounts of spectra in order to explore the evolution of our Galaxy. Until the release of the first substantial catalog of Gaia (estimated to be available in

\footnotetext{
24 The stellar overall metallicity is defined as

$[\mathrm{M} / \mathrm{H}]=\log (N(M) / N(H))_{\star}-\log (N(M) / N(H))_{\odot}$, where $N$ represents the number density and $M$ all the elements heavier than He.
} 
early 2017), the already current main large spectroscopic surveys of the Milky Way are the Sloan Extension for Galactic Understanding and Exploration (SEGUE), the RAdial Velocity Experiment (RAVE), the APO Galactic Evolution Experiment (APOGEE), the LAMOST Experiment for Galactic Understanding and Exploration (LEGUE), the GALactic Archaeology with HERMES (GALAH), and the Gaia-ESO Survey (GES).

RAVE $^{25}$ began observations in 2003, and since then it has released three data releases (hereafter DR): DR1 in 2006, DR2 in 2008, and DR3 in 2011 (Steinmetz et al. 2006; Zwitter et al. 2008; Siebert et al. 2011b). Furthermore, three catalogs with spectrophotometrically derived distances were published (Breddels et al. 2010; Zwitter et al. 2010; Burnett et al. 2011), and one catalog with abundances for the individual elements magnesium $(\mathrm{Mg})$, aluminum $(\mathrm{Al})$, silicon $(\mathrm{Si})$, calcium $(\mathrm{Ca})$, titanium (Ti), iron (Fe), and nickel (Ni; Boeche et al. 2011). RAVE is a magnitude-limited survey of stars randomly selected in the southern celestial hemisphere. The original design was to only observe stars in the interval $9<I<12$, but the actual selection function includes stars both brighter and fainter (see Section 2). The spectra are obtained from the $6 \mathrm{dF}$ facility on the $1.2 \mathrm{~m}$ Anglo-Australian Observatory's Schmidt telescope in Siding Spring, Australia, where three field plates with 150 robotically positioned fibers are used in turn. The effective resolution of RAVE is $R=\lambda / \Delta \lambda \sim 7500$, and the wavelength range coverage is around the infrared ionized Calcium triplet (IR Ca II, $\lambda \lambda 8410-8795$ ), one of the widely used wavelength ranges for Galactic archaeology. Up to now, previous RAVE catalogs have released 83,072 radial velocities for 77,461 stars, and 41,672 sets of atmospheric parameters for 39,833 stars. These produced many valuable studies.

Seabroke et al. (2008) used the symmetry of the velocity distributions to rule out the presence of the Sagittarius stream or the Virgo overdensity in the solar neighborhood. Recently, RAVE data allowed Williams et al. (2011) to discover the Aquarius stream and Antoja et al. (2012) to identify additional moving groups in the Galactic disk, consistent with dynamical models of the effects of the bar and the spiral arms. Pasetto et al. (2012a, 2012b) used RAVE data in order to constrain the solar motion relative to the local standard of rest, the rotational lag of the thick disk component, and the components of the thin disk velocity ellipsoids in the solar neighborhood. Additionally, Siebert et al. (2011a, 2012) measured the mean galactocentric radial velocity $(\mathrm{RV})$ of stars in the extended solar neighborhood and constrained the parameters of Milky Way spiral structure. Williams et al. (2013) identified differences in the velocity distribution between the north and the south of the Galactic plane with indications of a rarefaction-compression pattern, suggestive of wave-like behavior. Furthermore, Ruchti et al. (2011), Wilson et al. (2011), and Fulbright et al. (2010) studied the chemo-dynamical information of the thick disk and metalpoor stars of the Galaxy, whereas Matijevič et al. (2010, 2011) used RAVE to study single-lined and double-lined binary stars.

Here we present the new DR4 catalog, which includes 482,430 spectra. In order to obtain the atmospheric parameters, an updated version of the Kordopatis et al. (2011a) pipeline is used, which combines the DEGAS decision-tree method (DEcision tree alGorithm for AStrophysics; Bijaoui et al. 2012) and the MATISSE projection algorithm (MATrix Inversion for Spectral SynthEsis; Recio-Blanco et al. 2006) and takes into account, for the first time, the Two Micron All Sky Survey

\footnotetext{
25 http://www.rave-survey.org
}

(2MASS) photometric information. This allows us to treat more efficiently the spectral degeneracies than the maximum a posteriori method used in the previous data releases, reducing parameter combinations that were found in astrophysically nonjustified parts of the $\left(T_{\text {eff }}-\log g\right)$ space.

Furthermore, this DR4 catalog takes advantage of a multitude of new calibration data sets that have been collected recently in order to obtain reliable metallicities. The calibration libraries consist of RAVE and RAVE-like spectra for which there are parameter determinations derived from high-resolution spectroscopy, as well as stars selected from open and globular clusters of well-known metallicities. In addition to the stellar atmospheric parameters published for the newly observed targets, these calibration spectra and the new pipeline have also been applied in order to re-estimate and re-calibrate the parameters from the previous data releases. Then, given the new effective temperatures, surface gravities, and metallicities, individual abundances are also computed for the entire data set, using an updated version of the Boeche et al. (2011) chemical pipeline. Finally, new distances are also inferred, using the methods presented in Zwitter et al. (2010) and Binney et al. (2013).

The paper is structured as follows: first, in Section 2 we present the new input catalog of RAVE, and in Section 3 we present the pipeline that is used in order to obtain the atmospheric parameters, by recalling the basic equations and the updates of the MATISSE and DEGAS algorithms that are in the core of this new parameterization pipeline. Then, in Section 4, we show which calibration data sets are used and discuss how the calibration relation is obtained. Section 5 presents the updates on the chemical pipeline that is used in order to measure the individual abundances. Based on the presented pipelines and the calibration relation that is established, we present in Section 6 the DR4 atmospheric parameter catalog, as well as a comparison with the previous DR3 parameters, in particular for the metallicities. Sections 7, 8, 9, and 10 present the DR4 catalog for the proper motions, the radial velocities, and the stellar distances, as well as a description of the AAVSO Photometric All-Sky Survey (APASS) photometry, which is recommended to be used as it becomes available. Finally, Section 11 provides a summary.

\section{NEW RAVE INPUT CATALOGS}

The RAVE wavelength window of 8410-8795 $\AA$ implies that an I-band selection of the targets is the most appropriate. However, when RAVE started observing in 2003 April, there was no southern sky $I$-band photometric survey spanning the RAVE magnitude range. Therefore, the original input catalog was constructed by deriving I magnitudes from Tycho2 photometry and filling in Tycho-2's incompleteness at the faint end of the RAVE magnitude range with SuperCOSMOS photographic I magnitudes (see DR1 paper for more details). The DEep Near Infrared Survey of the Southern Sky (DENIS) DR2 was available in 2005 May (DENIS Consortium 2003), which included Gunn-I photometry at $0.82 \mathrm{~nm}$, but did not have sufficient coverage (55\% of the southern sky) to be used as the basis for a new RAVE input catalog. RAVE DR1, DR2, and DR3 were solely observed from the original input catalog. DR3 was the last release to be solely observed from the original input catalog, thereby concluding the pilot survey.

DENIS DR3 was available in 2005 September (DENIS Consortium 2005) and did have sufficient coverage (80\% of the southern sky) to be used as the basis for a new RAVE 


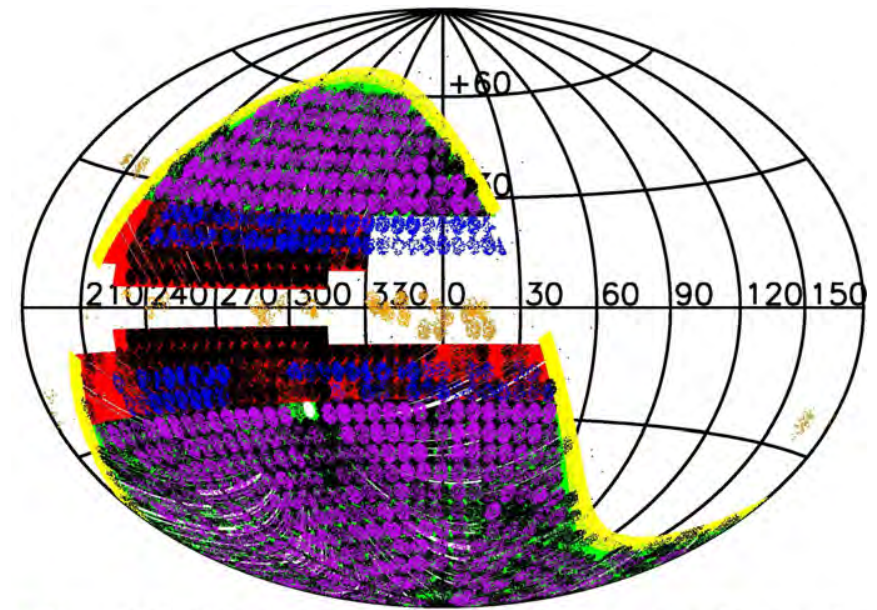

- New DENIS input catalogue: $|b|>25^{\circ}$, no colour cut

- New DENIS input catalogue: $|\mathrm{b}|<25^{\circ}, \mathrm{J}-\mathrm{K}>0.5$

New 2MASS input catalogue: $0^{\circ}<\delta<5^{\circ}$, no colour cut

RAVE DR4 from old input cotalogue

RAVE DR4 from interim input catalogue

RAVE DR4 from new input catalogue

Special fields

Figure 1. Aitoff projection of Galactic coordinates of the new input catalogs, color-coded into no color cut and color cut samples. Overlaid are the RAVE DR4 stars, color-coded according to their source input catalog. Their pattern is due to the $6 \mathrm{dF}$ field of view (5.9). The original input catalog was observed with field center coordinates separated by 5.7 . The new input catalog was observed with field center coordinates separated by 5.0 .

(A color version of this figure is available in the online journal.)

input catalog. DENIS entries within the RAVE magnitude range $(9<I<12 \mathrm{mag})$ were selected, including saturated entries (DENIS saturates at $I<9.8 \mathrm{mag}$ - see later discussion). Entries with $I$ magnitude error $=1$ indicate a non-estimated error, so these entries were rejected. Then the remaining entries were cross-matched with 2 MASS using a 1 arcsec box search. This search region was chosen because both DENIS and 2MASS were calibrated on the USNO-A2.0 catalog, which has an astrometric accuracy of 0.5 arcsec. The nearest DENIS DR3 catalog entry to a 2 MASS star provided the $I$ magnitude for that star (2MASS does not include $I$-band photometry). DENIS catalog entries not within a 1 arcsec search box of a 2 MASS star were rejected.

At the edges of the DENIS CCD detector, both the astrometry and photometry become less accurate. For each different scan of DENIS strip overlap regions, the catalog reports every detection of a source. If a star in this region is imaged more than once, each detection is included in the catalog because, although the multiple detections have almost identical magnitudes and positions, they cannot be associated with the same source. Crossmatching DENIS with 2MASS not only removes these multiple detections from the new input catalog but also provides more accurate astrometry, leading to fiber placement better matching stellar positions on the sky, which results in higher signal-tonoise spectra. DENIS includes spurious sources due to charge bleeding and diffraction spikes, which do not have matches in 2MASS and so are removed from the new DENIS input catalog, increasing its efficiency.

Comparison of Figure 1 with Figure 17 of Siebert et al. (2011b) shows that, in addition to covering the sky area of the original RAVE input catalog, the new DENIS input catalog has the major new feature of extending to lower Galactic latitudes (b). The aim of the extension toward the Galactic anti-rotation direction $\left(225^{\circ}<l<315^{\circ}, 5^{\circ}<|b|<25^{\circ}\right)$ is to observe the outer Galactic disk. Distances probed at the faint magnitude limit $(I=12 \mathrm{mag})$ are expected to be up to $\sim 5 \mathrm{kpc}$ for K-type giants (assuming $M_{I}=+1 \mathrm{mag}$ and no extinction). Therefore, at low Galactic latitudes in this direction, giants just reach the nominal "edge" of the stellar disk. Symmetric sampling about the Galactic plane will constrain the disk warp and flare. Distances probed at $I=12 \mathrm{mag}$ are only up to $\sim 400 \mathrm{pc}$ for G-type dwarfs (assuming $M_{I}=+4 \mathrm{mag}$ ). Therefore, the most efficient way to observe the outer disk is to apply a color cut ( $J-K>0.5 \mathrm{mag}$ ) in this region to avoid observing G-type dwarfs and preferentially observe K-type giants. The Besançon Galactic model (Robin et al. 2003) predicts that in the RAVE magnitude range at $J-K>0.5 \mathrm{mag} 70 \%$ of stars are KM-type giants and $30 \%$ are KM-type dwarfs. Although DENIS includes $J$ and $K$ photometry, the color cut is performed using 2MASS $J$ and $K$. This is because of the aforementioned edge effect on the DENIS CCDs and due to higher levels of sky image noise in DENIS than 2MASS. The noise comes from the thermal infrared background radiation emitted by the instrument itself. 2MASS optics avoid this by including a cold stop, which DENIS optics do not have.

After observing the new DENIS input catalog from 2006 to 2010, more targets closer to the Galactic plane were required to maintain RAVE's observing efficiency due to sky regions not always being observable from the UK Schmidt Telescope at different times of the year. The aim was still to target red giants by selecting $J-K>0.5 \mathrm{mag}$, thereby rejecting young foreground stars, which have weak Paschen lines that yield less accurate radial velocities. This selection works with reddening of $E(B-V)<0.35 \mathrm{mag}$. Therefore, to preserve a homogeneous selection function with this color cut, the new DENIS input catalog was extended closer to the Galactic plane in regions where $E(B-V)<0.35 \mathrm{mag}$. The northern Galactic hemisphere of the Galactic bulge has $E(B-V)>0.35 \mathrm{mag}$, and so $b<25^{\circ}$ at $l>330^{\circ}$ and $l<30^{\circ}$ is not included in the new extended DENIS input catalog. $10^{\circ}<b<25^{\circ}$ at $l<225^{\circ}$ and at $315^{\circ}<l<330^{\circ}$ has $E(B-V)<0.35 \mathrm{mag}$ and so is included. In the southern hemisphere of the bulge, $-10^{\circ}<b<-25^{\circ}$ at $l<225^{\circ}, l>315^{\circ}$, and $l<30^{\circ}$ all have $E(B-V)<0.35$ mag and so are included.

DR4 includes observations of the interim input catalog, which extended the original input catalog from $|b|<25^{\circ}$ to $|b|<15^{\circ}$ at all $l$. These observations were taken before the new DENIS input catalog was available, which is why they sample the northern bulge (blue dots in Figure 1) outside of the new extended DENIS input catalog color cut footprint (red dots in Figure 1). However, there are many observations of the interim input catalog (blue dots in Figure 1) within the color cut footprint. It is important to note that these do not include the color cut and so include all colors. These fields can be identified using the Galactic coordinates in Figure 1 and obsdate $\leqslant 20060312$. The special fields outside of the color cut footprint (orange dots in Figure 1) are specific science and calibration fields and do not include the color cut.

More bright targets north of the celestial equator $\left(\delta \leqslant 5^{\circ}\right)$ were required, again, to maintain RAVE's observing efficiency in bright time. DENIS's sky coverage is $\delta \leqslant 2^{\circ}$. Therefore, to extend the input catalog to $\delta=5^{\circ}$ required DENIS $I$ to be constructed from 2 MASS $J$ and $K$ using

$$
I_{\mathrm{DENIS}}=J_{2 \mathrm{MASS}}+0.054+1.18\left(J_{2 \mathrm{MASS}}-K_{2 \mathrm{MASS}}\right),
$$

where $\Delta_{\text {DENIS }}=0.15$ mag. This was done for 2MASS sources with $0^{\circ} \leqslant \delta \leqslant 5^{\circ}$ and $0^{\mathrm{h}}<\alpha<6^{\mathrm{h}}, 7^{\mathrm{h}} 30<\alpha<17^{\mathrm{h}}$, and 


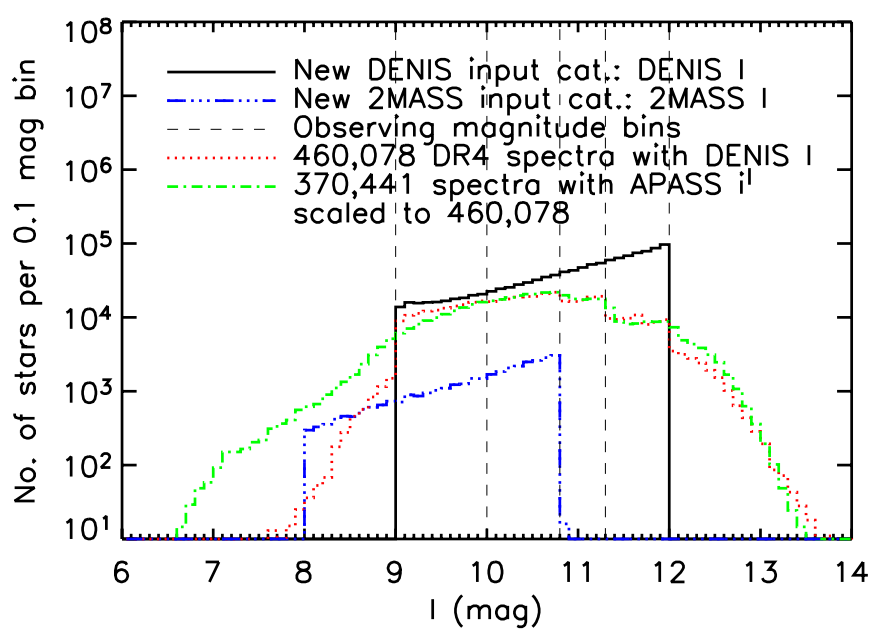

Figure 2. Histogram comparing the new input catalogs as a function of $I$ magnitude and the selection functions of all the observed RAVE stars as a function of $I$ magnitude and as a function of APASS $i^{\prime}$ magnitude, which is similar but not identical to DENIS $I$.

(A color version of this figure is available in the online journal.)

$19^{\text {h }} 30<\alpha<24^{\text {h }}$ (yellow dots in Figure 1) to avoid the Galactic plane. The 2MASS input catalog spatially overlaps the DENIS input catalog by $\Delta \delta=3^{\circ}$ because DENIS's sky coverage is not complete. The long white areas within the colored regions in Figure 1 are one or more slots (12 $\operatorname{arcmin}$ in $\alpha$ by $30^{\circ}$ in $\delta$ ), where the observed DENIS strips filling these slots are missing from DENIS DR3. The small white areas within the colored regions are one or more missing DENIS images (12 $\operatorname{arcmin} \times$ 12 arcmin). The white circular region within the colored regions is the southern equatorial pole, which is not observed by DENIS. The width of the missing DENIS strips and images is $3.5 \%$ of the $6 \mathrm{dF}$ field of view and so does not pose a fiber configuration problem. Its effect on RAVE's random selection of targets should be negligible. It is important to note that observations from the 2MASS input catalog at $|b|>25^{\circ}$ do not include the color cut and so include all colors. Figure 1 shows that there are only three DR4 fields that spatially overlap the DENIS input catalog color cut footprint and the 2MASS input catalog footprint and so have mixed selection functions.

Figure 2 compares the new input catalogs and the number of observed DR4 spectra as a function of $I$ magnitude. It emphasizes that the observed DR4 spectra have not exhausted the new DENIS input catalog overall and so are not complete with respect to DENIS overall, although individual fields may be complete. Indeed, the new DENIS input catalog is only complete where DENIS has sky coverage. DR4's completeness with respect to 2MASS is shown in Figure 3. Figure 2 also shows that the 2MASS input catalog extends the bright limit of the survey to $I=8 \mathrm{mag}$. Each $6 \mathrm{dF}$ field setup only selects new input catalog targets from one of the following four magnitude bins: $9.0<I<10.0(8.0<I<10.0$ for the new 2MASS input catalog), $10.0<I<10.8,10.8<I<11.3$, and $11.3<I<12 \mathrm{mag}$, which are visible in Figure 2. This minimizes the magnitude range within any one $6 \mathrm{dF}$ field setup to be within a bin, meaning exposure times can be scaled more appropriately for all the targets in the same field. Each field

\section{$9.0<\mathrm{I}_{2 \text { MASS }}<10.0$}
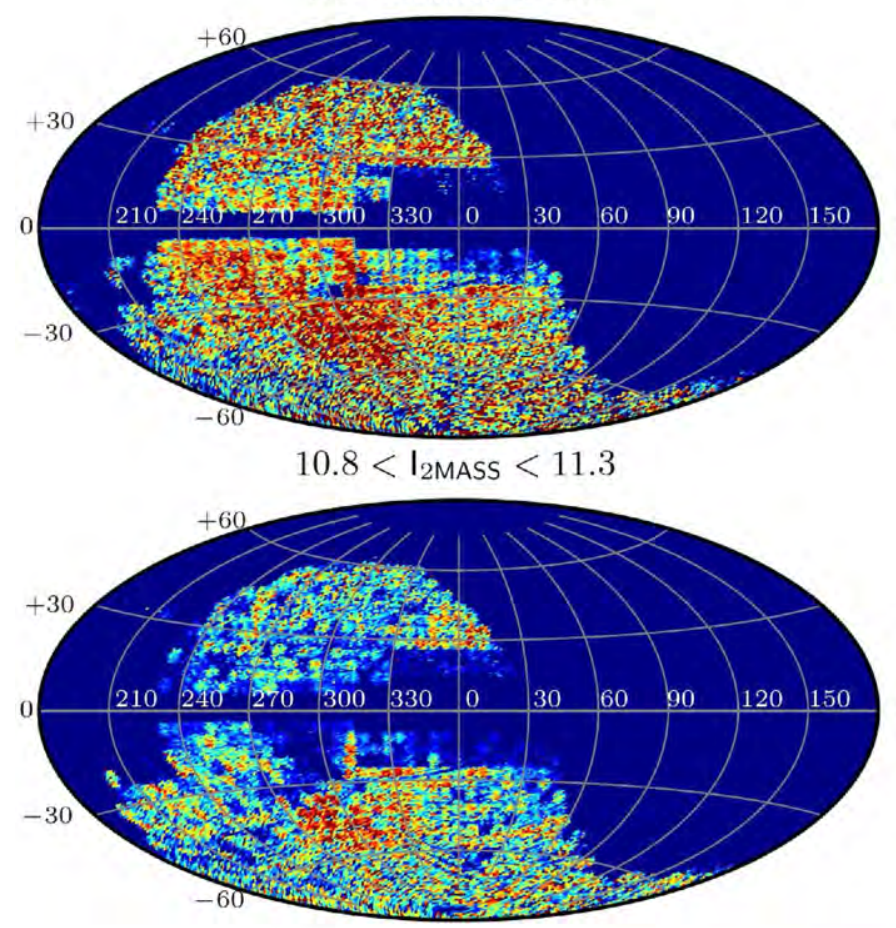

$10.0<\mathrm{I}_{2 \text { MASS }}<10.8$

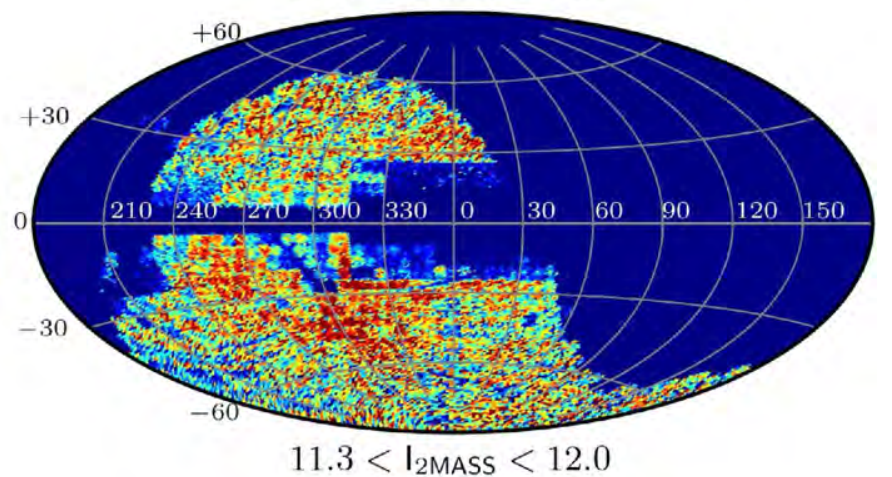

$11.3<\mathrm{I}_{2 \text { MASS }}<12.0$

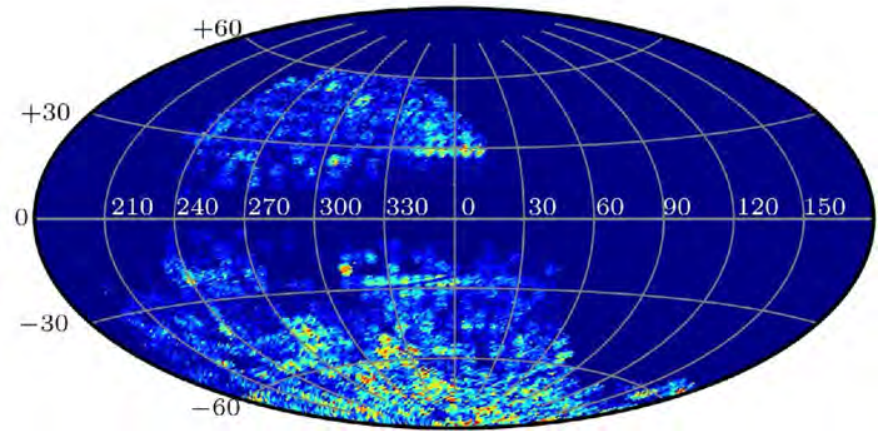

0.0
0.5

$N_{\text {RAVE }} / N_{2 \text { MASS }}$

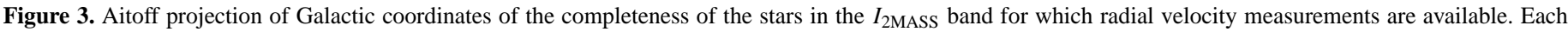

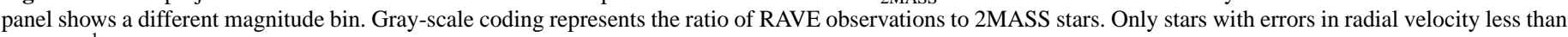
$10 \mathrm{~km} \mathrm{~s}^{-1}$ are shown.

(A color version of this figure is available in the online journal.) 


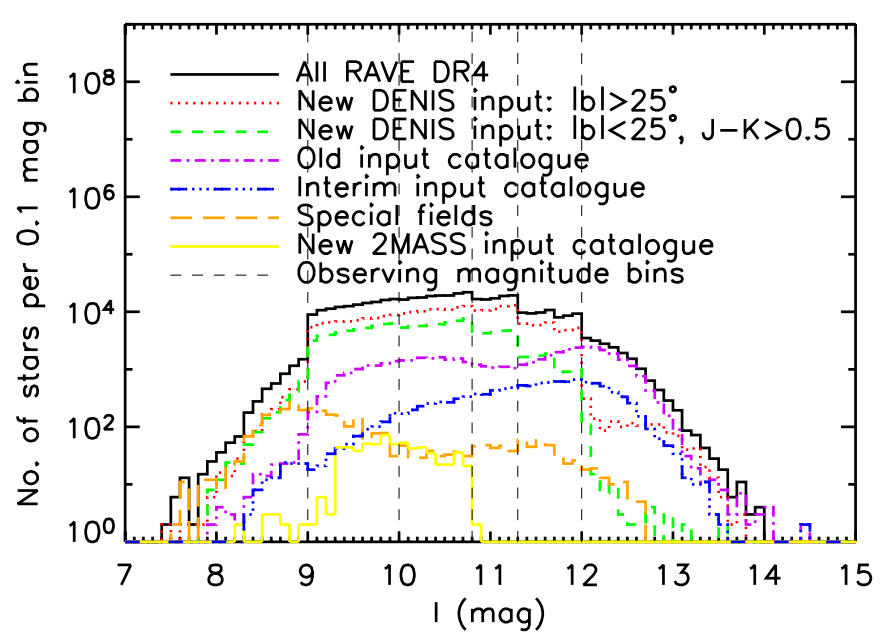

Figure 4. Histogram decomposing DR4 into its constituent input catalogs as a function of $I$ magnitude. Note that this is either DENIS I or 2MASS constructed DENIS $I$ and so is not reliable at $I<9.8$ mag due to DENIS $I$ saturation.

(A color version of this figure is available in the online journal.)

setup is a random selection of unobserved targets within these bins (apart from designed repeat observations). Any spectrum within a $6 \mathrm{dF}$ field setup can be adjacent to any other in the same setup on the CCD, but the bins limit the magnitude difference, which also minimizes fiber cross-talk.

Figure 2 compares the selection functions of the new input catalogs and the observed DR4 stars. The input catalogs have step functions at their bright and faint ends. However, the observed DR4 stars do not have step functions but extend to brighter and fainter than the input catalogs. Because DENIS saturates at $I<9.8 \mathrm{mag}$, the selection function of RAVE's new input catalog and observations need to be compared to an $I$-band survey that does not saturate in the RAVE magnitude range. The recent advent of the APASS (see Section 10) means that this can now be explored. Future data releases will be supplemented with APASS photometry. At the time of writing, APASS $i^{\prime}$ (internal release DR7) was available for 370,441 spectra and is plotted in Figure 2, scaled to the number of DR4 spectra. It shows that the distribution of APASS $i^{\prime}$ approximately agrees with the distribution of DENIS $I$ at the faint end but extends to much brighter magnitudes at the bright end. This is because DENIS saturates at $I<9.8 \mathrm{mag}$, which means some sources are actually $I<9$ mag.

The overall selection function of DR4 is more clearly seen in Figure 4. It has a complex shape due to the observing strategy and because DR4 includes all RAVE observations up to the end of 2012, which have been selected from four different RAVE input catalogs. Numerically, DR4 is dominated by stars selected from the new DENIS input catalog $\left(|b|>25^{\circ}\right)$. This means the observing magnitude bins are visible in the DR4 selection function. It also means that overall the new input catalog observations have filled in the incompleteness of the old input catalog (mainly due to Tycho-2), thus reducing the presence of its subtle selection biases in DR4, compared to DR1, DR2, and DR3. Therefore, overall RAVE DR4 is more representative of the underlying Galactic stellar populations than previous DRs, with the exception of $|b|<25^{\circ}$, which deliberately targets giants with $J-K>0.5$ mag. Nevertheless, on a field-byfield basis, Galactic science still requires care to account for the various selection biases introduced by the inhomogeneous photometry used to derive some of the input samples (detailed in the DR1 paper). Care is required if stars are selected from DR4 using their observation date as the selection function changes as a function of time: old and interim input catalog obsdate $\leqslant 20060312$; new DENIS input catalog obsdate > 20060312; new 2MASS input catalog obsdate $>20120128$. There may also be a stellar position bias because the $6 \mathrm{dF}$ fiber positioner software avoids putting targets too close to each other and also avoids crossing of fibers.

Figure 4 shows that the faint tail of the DR4 magnitude distribution is dominated by the old input catalog. This tail is fainter than the nominal selection of $I<12 \mathrm{mag}$ due to SuperCOSMOS photographic I magnitudes being saturated. The twin peaks of the old input catalog are due to the merging of Tycho- 2 and SuperCOSMOS. The interim input catalog also includes Tycho-2 and SuperCOSMOS but weights their relative numbers to achieve a single peak in its magnitude distribution. The special fields are identified by being away from the main data. However, there are many calibration and science stars within the main footprint of the survey and so are classed as stars observed from the new input catalog. These dominate the bright tail of the DR4 magnitude distribution in Figure 4 and also contribute to the faint tail. The old and interim input catalogs contribute to the bright tail because their bright magnitudes were constructed from Tycho- $2 B_{T}$ and $V_{T}$.

To summarize, DR4 has no kinematic bias and no overall photometric bias. The notable exception is the color criterion ( $J-K>0.5 \mathrm{mag}$ ) to deliberately target giants at $|b|<25^{\circ}$ (except where $\delta>0^{\circ}$ ). No other color cuts exist in the data. However, on a field-by-field basis, subtle selection biases may still be present.

\section{THE NEW PIPELINE FOR THE STELLAR PARAMETERS}

The wavelength region $\lambda \lambda 8410-8795$ is often used for Galactic archaeology purposes, as for instance with the multi-fiber spectrographs of ESO FLAMES-Giraffe at the LR8 and HR21 setups, and the Gaia-RVS. Indeed, it is a spectral region with relatively few telluric absorptions that exhibits many iron and $\alpha$-element lines, in particular the prominent $\mathrm{Ca}$ II triplet $(\lambda=8498.02 \AA, 8542.09 \AA, 8662.14 \AA)$. This feature is still visible at low signal-to-noise-ratio $(\mathrm{S} / \mathrm{N})$ and low metallicity, and as a consequence, it allows relatively easily to have RV measurements and metallicity estimations for any type of spectrum.

\subsection{Spectral Degeneracies in the Infrared Ionized Calcium Triplet Wavelength Range}

The recent work of Kordopatis et al. (2011a, hereafter K11) on spectra with a resolution $R \leqslant 10,000$ has shown that the $\mathrm{Ca}$ II wavelength range suffers from spectral degeneracies that, if not appreciated, can introduce serious biases in spectroscopic surveys that use automated parameterization pipelines. These degeneracies are mostly important for cool main-sequence stars and stars along the giant branch. On the one hand, the spectral signatures that are used to determine the surface gravities for the main-sequence stars are insensitive to small $\log g$ variations, hence reducing the accuracy of the measurement of that parameter. On the other hand, the spectra of stars along the giant branch can be identical for different sets of parameters. In this case, the degeneracy is due to the fact that the spectral signatures sensitive to variations of effective temperature, surface gravity, and metallicity are the same. The degeneracy works as follows: the spectrum corresponding 
to a given $T_{\text {eff }}, \log g$, and $[\mathrm{M} / \mathrm{H}]$ is almost identical to a spectrum corresponding to a lower (higher) temperature, lower (higher) surface gravity, and lower (higher) metallicity.

Automated parameterization pipelines try to find the model template that minimizes the distance function, defined as the difference between the observation and a set of reference (model) spectra. In the case where not enough information is available in the data, the distance function can become non-convex, and secondary minima can appear, in which the parameterization algorithms can falsely converge. If these secondary minima are close in the parameter space, the resulting parameter estimation will have a large scatter around the true value, whereas if they are distant in the parameter space, the algorithms might converge randomly to one or the other according to where the noise is placed in the spectrum.

In K11 it has been shown that for low- and medium-resolution spectra around the IR Ca II, decision-tree methods manage to better find the absolute minimum of the distance function, compared to other algorithms, like the projection methods (e.g., principal component analysis) or the ones trying to solve an optimization problem (e.g., minimum $\chi^{2}$ ), in particular when the $\mathrm{S} / \mathrm{N}$ is low. For that reason, the pipeline presented in $\mathrm{K} 11$ iteratively renormalizes the spectra and obtains the atmospheric parameters using a combination of a decision-tree algorithm called DEGAS (Bijaoui et al. 2012) and a projection method called MATISSE (Recio-Blanco et al. 2006), which allows us to better interpolate between the grid points.

Both DEGAS and MATISSE have a learning phase based on a nominal library of synthetic spectra (i.e., the templates), described in Section 3.4. Here we describe briefly the pipeline and the two algorithms, but we refer the reader to Recio-Blanco et al. (2006), Bijaoui et al. (2012), and Kordopatis et al. (2011a) for further details.

\subsection{DEGAS: An Oblique $k$-d Decision-tree Method for the Low-S/N Spectra, and for Re-normalizations}

In the limit of the sampling precision of a learning grid (i.e., the parameter steps), parameter estimation is a pattern recognition problem. The grid of synthetic spectra can be treated as a known set of patterns among which the observed spectra should be identified. The DEGAS algorithm is an oblique 3D decision tree (in our case $T_{\text {eff }}, \log g$, and $[\mathrm{M} / \mathrm{H}]$ ), for which the decisions result from the projection of the observations onto $N$ node vectors noted $D_{n}(\lambda)(n=1, \ldots, N)$. These node vectors are associated with a subset of spectra of the nominal library, in the sense that the result of the projection of an observed spectrum on that node will select half of the subset which most closely resembles the observation.

The recognition rules of DEGAS, at each node, are established during the learning phase as follows:

1. The mean vector $M(\lambda)$ of the flux values per pixel of all the reference spectra in the subset is computed.

2. For each reference spectrum $S_{j}(\lambda)$ associated with the node, the scalar product $c_{j}=\sum_{\lambda} S_{j}(\lambda) \cdot M(\lambda)$ is calculated. Let $\tilde{\mathrm{c}}$ be the median value of $c_{j}$.

3. The reference spectra are bisected in two new subsets, $T_{1}$ and $T_{2}$, according to the following criteria:

$S_{j}$ belongs to the subset $T_{1}$ if $c_{j} \leqslant \tilde{\mathrm{c}}$ $S_{j}$ belongs to the subset $T_{2}$ if $c_{j}>\tilde{\mathrm{c}}$.

4. The difference vector $D(\lambda)=M_{1}(\lambda)-M_{2}(\lambda)$ is determined, where $M_{1}(\lambda)$ and $M_{2}(\lambda)$ are the mean vectors of the flux values of each subset.
5. If the correlation coefficient between $M(\lambda)$ and $D(\lambda)$ is too small (typically, smaller than 0.999), the initial subset of reference spectra is re-separated by the hyperplane defined by $D(\lambda)$, iterating until convergence (going back to step 2, replacing $M(\lambda)$ by $D(\lambda)$ ).

When the previous procedure has converged for a particular node $n$, the final adopted projection node vector $D_{n}(\lambda)$ is determined, which will display the features that allow the separation of the subset of learning spectra at that node. The final median value $\tilde{c_{n}}$ of $c_{j}=\sum_{\lambda} S_{j}(\lambda) \cdot D_{n}(\lambda)$ that will allow us to make the decisions is also set.

In this way, the recognition tree is built, having $\log _{2}(N)$ levels, where $N$ is the number of spectra of the learning grid. At the lowest level nodes of the tree, only one training spectrum remains associated with each node. During the application phase, the target data $O_{i}(\lambda)$ pass through all the levels of the recognition tree, and a template is associated with it.

Of course, noise can induce misclassifications. The exploration of the branches, even if the decision threshold $\tilde{c_{n}}$ does not allow it, is accomplished thanks to an activation function on the directions of the tree. Let us consider

$$
u_{i}=\frac{c_{i}-\tilde{c_{n}}}{\sigma_{c_{i}}},
$$

where $c_{i}=\sum_{\lambda} O_{i}(\lambda) \cdot D_{n}(\lambda), \sigma_{c_{i}}=S_{f} \cdot \sqrt{\Sigma_{\lambda} D_{n}(\lambda)^{2}}$, and $S_{f}$ is an arbitrary constant chosen in order to explore optimally the branches. If $u_{i} \leqslant-k$, we decide that the correct direction is 1 . If $u_{i} \geqslant k$, the direction 2 is chosen. If $-k<u_{i}<k$, both directions are considered.

After the scanning of all the nodes, a subset of synthetic templates is selected, and their distances (in terms of the difference with the observed spectrum) are computed. Then, the parameters of the observed spectrum are determined by computing a weighted mean on the selected spectra, taking into account these distances, setting

$$
W_{i}^{n}=\left(1-\left[\sum_{\lambda} O_{i}(\lambda)-S_{n}(\lambda)\right]^{2}\right)^{p} .
$$

The value $p$ of the exponent is rather arbitrary. Following K11, where it was found that $p=64$ gave the best results, the same value was adopted in what follows.

DEGAS has a key role in the pipeline used for the RAVE DR4 analyses. Because of its pattern recognition approach, it is exploring more efficiently the parameter space than other mathematical methods and, as a consequence, is less affected by secondary minima in the distance function. Hence, given a roughly normalized spectrum at the rest frame, DEGAS is used in order to achieve a good normalization for the data, using the synthetic spectrum corresponding to the intermediate solution found.

Once DEGAS has converged on a set of parameters, the new parameters are used to re-normalize the spectrum given the intermediate solution template. The re-normalization process might need several iterations until the shape of the continuum stays unchanged from one normalization to another. The number of needed iterations may vary from 3 to 10 . In the case where the distance function has many secondary minima (low-S/N spectra and/or low-metallicity stars), the results of DEGAS at that stage are the most accurate among the other methods. Nevertheless, it has been shown in K11 that MATISSE manages to better 
interpolate between the grid points when the astrophysical information is sufficient in the spectra. Thus, once the shape of the continuum has converged, the mean $\mathrm{S} / \mathrm{N}$ per pixel is estimated in the same way as in Zwitter et al. (2008), and in the case of high-S/N spectra MATISSE is run to get more precise atmospheric parameters. The $\mathrm{S} / \mathrm{N}$ threshold at which MATISSE is run has been established in $\mathrm{K} 11$ to be $\mathrm{S} / \mathrm{N}=30 \mathrm{pixel}^{-1}$.

\subsection{MATISSE: A Projection Method for the High-S/N Regimes}

The MATISSE algorithm is a local multi-linear regression method. It estimates a $\hat{\theta}_{i}$ stellar atmospheric parameter $\left(i \equiv T_{\text {eff }}\right.$, $\log g,[\mathrm{M} / \mathrm{H}])$ by projecting the observed spectrum $O(\lambda)$ on a particular vector $B_{\theta}(\lambda)$ associated to a theoretical $\theta_{i}$ parameter, as follows:

$$
\hat{\theta_{i}}=\sum_{\lambda} B_{\theta_{i}}(\lambda) \cdot O(\lambda) .
$$

These vectors, called $B_{\theta}(\lambda)$ functions hereafter, are computed during a learning phase. They relate, in a quantitative way, the pixel-to-pixel flux variations in a spectrum to a given variation of the $\theta_{i}$ parameter. In the case where the $B_{\theta}(\lambda)$ are orthogonal, the effects due to each parameter affect the spectrum in an independent way, and hence the atmospheric parameters are derived accurately. When this is not the case (as in most applications), possible degeneracies in the parameter space can occur, causing a correlation of the parameter errors.

The $B_{\theta}(\lambda)$ functions are computed within a given range of parameters, from an optimal multi-linear combination of theoretical, synthetic spectra $S(\lambda)$, as follows:

$$
B_{\theta_{i}}(\lambda)=\sum_{j} \alpha_{i j} \cdot S_{j}(\lambda),
$$

where the $\alpha_{i j}$ factor is the weight associated with each synthetic spectrum $S_{j}(\lambda)$, in order to retrieve the $\hat{\theta}_{i}$ parameter. The weights are computed during the learning phase by applying Equation (4) to a subset of synthetic spectra. Thus, combining Equations (4) and (5), one obtains

$$
\Theta_{i}=C \alpha_{i},
$$

where $C=\left[c_{j j^{\prime}}\right]$ is the correlation matrix and $\Theta_{i}$ the vector of the parameters $\theta_{i}$ for all the considered spectra. The weights $\alpha_{i j}$ are then obtained by inverting the correlation matrix $C$. As explained in $\mathrm{K} 11$, a direct inversion of $C$ would take into account all the available spectral signatures, including the smallest ones. Nevertheless, in the case of noisy spectra some lines become insignificant and should hence be discarded from the analysis. In order to achieve that, we adopt here the same approach as in K11, which used the Landweber algorithm to iteratively invert $C$. By stopping the inversion procedure at different convergence values, smaller weights are then applied to the most insignificant lines, which allows the solution to be less affected by secondary minima in the distance function. Extensive analysis of the parameter space for spectra of different $\mathrm{S} / \mathrm{N}$ values allowed adoption of a different set of $B_{\theta}(\lambda)$ functions for different $S / N$ values and hence achieved enhanced accuracy in the parameter determination (see K11 for further details).

The convergence of the algorithm works as follows: if the projection of the observed spectrum on a set of $B_{\theta}(\lambda)$ gives results that are not within the parameter ranges for which these $B_{\theta}(\lambda)$ have been computed, then a new set of $B_{\theta}(\lambda)$ is used, centered on the previous solution. This step is repeated until the results stay within the parameter range of applicability of the projection vectors. In the case where the distance function is convex, less than five iterations are usually needed to reach the absolute minimum, but in some cases of degeneracy in the distance function, the algorithm might not converge (these spectra are then flagged and should not be used from the catalog; see Section 6.1).

As noted previously, MATISSE has the advantage of interpolating accurately between the learning grid points, achieving a good parameter estimation at the high-S/N regimes. Nevertheless, local projection methods such as MATISSE have, in general, two main disadvantages. The first consists in not exploring entirely the parameter space and hence being easily trapped in secondary minima of the distance function if there is a lot of noise in the spectrum. The second disadvantage is that it can extrapolate results outside the boundaries of the learning grid. These two undesired effects are attenuated with the parallel use of DEGAS. Indeed, as described in Section 3.2, DEGAS is first used to converge toward a sub-region of the parameter space. Then, for the lowest $\mathrm{S} / \mathrm{N}$ spectra or when the derived MATISSE parameters are outside the grid's limits, the results of DEGAS are adopted, and MATISSE is not implemented. ${ }^{26}$

\subsection{The Grid of Synthetic Spectra for the Learning Phase of the Pipeline}

The very high resolution grid computed in K11 has been convolved with a Gaussian kernel, in order to obtain a new synthetic library at $R=7500,{ }^{27}$ with a constant wavelength step of $0.35 \AA$ and covering the wavelength range of 8450.80-8746.55 $\AA$. In addition, the cores of the $\mathrm{Ca}$ II lines have been removed from the synthetic spectra, corresponding to 1 pixel for the first Ca II line and 2 pixels for the other two lines. This removal is justified since the cores of the lines are formed in the upper stellar atmospheric layers where some modeling assumptions like the local thermodynamical equilibrium (LTE) or hydrostatic equilibrium might not be valid hypotheses anymore. The flux disagreement with real spectra for these pixels can induce the algorithms to converge to false minima, and hence the cores must be discarded (see K11 for further details).

We recall that the synthetic library has been computed using the one-dimensional, LTE and hydrostatic equilibrium MARCS model atmospheres (Gustafsson et al. 2008), in combination with the Turbospectrum code (Alvarez \& Plez 1998). The atomic line list has been calibrated on high-S/N and high-resolution spectra of the Sun and Arcturus (Brault \& Neckel 1987; Hinkle et al. 2003), assuming the solar abundances of Grevesse (2008), except for $\mathrm{CNO}$, where we used the values of Asplund et al. (2005). The molecular line list includes $\mathrm{ZrO}$, TiO, VO, CN, C2, $\mathrm{CH}, \mathrm{SiH}, \mathrm{CaH}, \mathrm{FeH}$, and $\mathrm{MgH}$ lines with their corresponding isotopic variations (kindly provided by B. Plez).

The reference grid spans effective temperatures from $4000 \mathrm{~K}$ to $8000 \mathrm{~K}$ with a constant step of $250 \mathrm{~K}$ and surface gravities from 0.0 dex to 5.0 dex with a constant step of 0.5 dex. In addition, the library spans with a constant step of $200 \mathrm{~K}$ effective temperatures from $3000 \mathrm{~K}$ to $4000 \mathrm{~K}$ and surface gravities from 0.0 dex to $5.5 \mathrm{dex}$. As far as the metallicities are concerned,

\footnotetext{
26 The stars for which DEGAS parameters have been adopted after MATISSE has given parameters outside the grid boundaries are flagged as well, and should be used with caution.

27 We note that the effective resolution of RAVE can in reality vary from $6500<R<8500$, the changes being a function of both time and position on the CCD. Nevertheless, simple tests, degrading the $S^{4} N$ library (see Section 4.5) to $R \sim 6500,7500$, and 8500 and then analysis as if the spectra were at $R \sim 7500$, show that the effect on the parameter estimation was of the second order. We hence did not take into account these resolution changes for DR4. However, future data releases will implement these second-order effects.
} 
the number of grid models has increased compared to K11. Instead of having a variable metallicity step, ranging from 0.25 dex for the most metal-rich stars to 1 dex for the stars with $[\mathrm{M} / \mathrm{H}]<-3$ dex, the new grid has a constant metallicity step of $0.25 \mathrm{dex}$ for all the metallicities ranging from $[-5.0 ;+1.0]$ dex. These new spectra, whose atmospheric models did not exist in the MARCS database, have been linearly interpolated from the existing synthetic spectra.

One of the noticeable differences between previous data releases and DR4 is that, in this work, only three parameters are independent in the grid: the effective temperature, $T_{\mathrm{eff}}$, the logarithm of the surface gravity, $\log g$, and the overall metallicity, $[\mathrm{M} / \mathrm{H}]$. This restriction decreases the number of the secondary minima of the distance function, hence increasing the accuracy of the parameter derivation. It should be noted though that the microturbulent velocity $(\xi)$ is not constant within the grid, but is changed in lock-step with the gravity of the stars. Dwarfs $(\log g \geqslant 3.5$ dex) have a microturbulent velocity of $\xi=1 \mathrm{~km} \mathrm{~s}^{-1}$, whereas giants have $\xi=2 \mathrm{~km} \mathrm{~s}^{-1}$. Abundances of the $\alpha$-elements ${ }^{28}$ are also changed systematically with metallicity, being scaled on the iron abundance, $[\mathrm{Fe} / \mathrm{H}]$, following the standard $\alpha$-enhancement found for the metal-poor stars of the Milky Way (thick disk and halo):

$$
\begin{aligned}
& \text { 1. }[\alpha / \mathrm{Fe}]=0.0 \text { dex for }[\mathrm{Fe} / \mathrm{H}] \geqslant 0.0 \operatorname{dex} \\
& \text { 2. }[\alpha / \mathrm{Fe}]=-0.4 \times[\mathrm{Fe} / \mathrm{H}] \text { dex for }-1 \leqslant[\mathrm{Fe} / \mathrm{H}] \leqslant 0 \operatorname{dex} \\
& \text { 3. }[\alpha / \mathrm{Fe}]=+0.4 \text { dex for }[\mathrm{Fe} / \mathrm{H}] \leqslant-1.0 \operatorname{dex} .
\end{aligned}
$$

In addition, spectra for which the parameter combinations did not correspond to realistic astrophysical stars have been removed from the learning grid and hence from the solution space as well. To minimize the importance of our astrophysical priors in the derived parameters, we removed only the templates with $\log g=5 \mathrm{dex}$ and $T_{\text {eff }}>6250 \mathrm{~K}$, those with $T_{\text {eff }} \leqslant 4250 \mathrm{~K}$ and $4 \leqslant \log g \leqslant 3 \mathrm{dex}$, as well as all stars with $[\mathrm{M} / \mathrm{H}] \leqslant$ $-3 \mathrm{dex}, T_{\text {eff }} \leqslant 4000 \mathrm{~K}$, and $\log g \leqslant 4$ dex. These criteria correspond to excluding very young stars with extremely metalpoor abundances (age $<0.5 \mathrm{Gyr}$ and $[\mathrm{Fe} / \mathrm{H}]<-2.5 \mathrm{dex}$ ), as well as old stars that are extremely metal-rich (age $>14 \mathrm{Gyr}$ and $[\mathrm{Fe} / \mathrm{H}]>0.75 \mathrm{dex})$. The final grid contains 3580 spectra of 839 pixels each.

Based on this grid, a subset of reference models can be selected, according to the additional information that is available for each data set to be treated. In the case of RAVE, the 2MASS photometric information that is available for the observed targets is used to exclude some parameter combinations from the solution space corresponding to derived temperature ranges which are grossly inconsistent with the photometric color. In practice, the RAVE spectra have been separated into four different color ranges. Then, according to their $2 \operatorname{MASS}\left(J-K_{S}\right)$ color, a set of solutions has been imposed as soft photometric priors for every analyzed spectrum, defining:

$$
\begin{aligned}
& \text { 1. }\left(J-K_{s}\right)>0.75 \Rightarrow T_{\text {eff }}<4500 \mathrm{~K} \\
& \text { 2. } 0.4<\left(J-K_{s}\right)<0.75 \Rightarrow 3750<T_{\text {eff }}<6000 \mathrm{~K} \\
& \text { 3. }\left(J-K_{s}\right)<0.4 \Rightarrow T_{\text {eff }}>5250 \mathrm{~K} \text {. }
\end{aligned}
$$

The few stars for which no 2MASS photometry was available (less than 2\%) form a fourth category for which there is no prior on the solution space.

The above-mentioned effective temperature ranges have been determined by requesting a color-magnitude diagram of the

\footnotetext{
28 The chemical species considered as $\alpha$-elements are $\mathrm{O}, \mathrm{Ne}, \mathrm{Mg}, \mathrm{Si}, \mathrm{S}, \mathrm{Ar}$, $\mathrm{Ca}$, and $\mathrm{Ti}$.
}

Galaxy from the web interface ${ }^{29}$ of the Padova database with the 2MASS photometric system. Then, for the above three color ranges we inferred the full range of effective temperatures of the simulated stars and increased these limits by $\pm 500 \mathrm{~K}$. We note that the effective temperature bins have been made deliberately large, because neither the photometric errors nor the extinction have been taken into account when separating the spectra into color bins.

\subsection{Computation of the Internal Uncertainties}

The total uncertainty of the pipeline for a given star is the quadratic sum of its internal and external errors. The internal uncertainties relate the capacity of an algorithm to treat spectral degeneracies and $\mathrm{S} / \mathrm{N}$, whereas external uncertainties concern the difference between the template synthetic spectra and the true stellar spectra (see Section 4.6).

Following K11, the internal uncertainties of the algorithm have been estimated by computing a set of spectra of realistic Galactic populations. Based on the Besançon Galactic model, a simulated catalog of stars toward three different Galactic directions (Galactic center, north Galactic pole, and intermediate Galactic latitudes) has been constructed, from which $10^{4}$ stars have been randomly selected to be our realistic Galactic sample. In addition, in order to explore different metallicity regimes, each star has been replicated in the catalog, with its partner having reduced (by -0.75 dex) metallicity. The $2 \times 10^{4}$ synthetic spectra corresponding to these parameter combinations have been computed thanks to the interpolation capabilities of MATISSE, and four different values of white Gaussian noise were added to them $\left(\mathrm{S} / \mathrm{N}=100,50,20,10 \mathrm{pixel}^{-1}\right)$. Given these final $8 \times 10^{4}$ spectra, the pipeline was run in order to retrieve the associated errors.

In order to simulate the way the RAVE spectra are analyzed, the pipeline was run twice: once without any photometric prior (see Table 1), and once by imposing soft priors (see Table 2), based on their temperatures. These priors have been selected in order to be similar with the ones that are applied in the analysis of the RAVE spectra (see Section 3.4). The error values for different stellar types, presented in Tables 1 and 2, have been computed as the 70th percentile of the error distribution. Indicative atmospheric parameter uncertainties for typical thin disk dwarfs, thick disk dwarfs, and halo giants are also given in the last three lines of these tables. A comparison of the uncertainty values with or without photometric priors shows that when the spectral degeneracy is important, the applied soft priors improve significantly the associated uncertainties (see, for example, the hot, metal-poor dwarfs). In addition, it has been noticed, as expected, that the use of the soft photometric priors improves the 90th percentile of most of the stellar categories considered in these tables.

The way the internal errors are associated with a specific parameter estimation is as follows: once the pipeline has converged toward a set of parameters, the final $\mathrm{S} / \mathrm{N}$ is computed as in Zwitter et al. (2008), utilizing the associated solution template. According to the $\mathrm{S} / \mathrm{N}$, the stellar type, the luminosity class, its metallicity, and the use or not of 2MASS photometric prior, the equivalent internal error estimations in Table 1 or Table 2 are adopted. We note that this approach does not optimally take into account the properties of the distance function and hence the degeneracies. Nevertheless, we find that

\footnotetext{
29 http://stev.oapd.inaf.it/cmd
} 
Table 1

Internal Errors after Re-normalizations without Photometric Priors

\begin{tabular}{|c|c|c|c|c|c|c|c|c|c|c|c|c|}
\hline \multirow[t]{2}{*}{$\mathrm{S} / \mathrm{N}\left(\mathrm{pixel}^{-1}\right)$} & \multicolumn{4}{|c|}{$T_{\text {eff }}(\mathrm{K})$} & \multicolumn{4}{|c|}{$\log g(\operatorname{dex})$} & \multicolumn{4}{|c|}{$[\mathrm{M} / \mathrm{H}](\mathrm{dex})$} \\
\hline & 100 & 50 & 20 & 10 & 100 & 50 & 20 & 10 & 100 & 50 & 20 & 10 \\
\hline K II-IV, $[\mathrm{M} / \mathrm{H}]>-0.5 \mathrm{dex}$ & 72 & 76 & 117 & 201 & 0.12 & 0.13 & 0.20 & 0.48 & 0.08 & 0.08 & 0.10 & 0.23 \\
\hline K II-IV, $-1<[\mathrm{M} / \mathrm{H}]<-0.5 \mathrm{dex}$ & 62 & 85 & 133 & 302 & 0.14 & 0.20 & 0.35 & 0.72 & 0.08 & 0.09 & 0.16 & 0.30 \\
\hline K II-IV, $-2<[\mathrm{M} / \mathrm{H}]<-1 \mathrm{dex}$ & 75 & 96 & 178 & 330 & 0.20 & 0.30 & 0.57 & 0.97 & 0.09 & 0.11 & 0.19 & 0.33 \\
\hline K II-IV, $[\mathrm{M} / \mathrm{H}]<-2 \operatorname{dex}$ & 78 & 105 & 184 & 382 & 0.31 & 0.40 & 0.76 & 1.26 & 0.09 & 0.10 & 0.20 & 0.37 \\
\hline G II-IV, $[\mathrm{M} / \mathrm{H}]>-0.5 \mathrm{dex}$ & 78 & 111 & 233 & 402 & 0.09 & 0.20 & 0.40 & 0.69 & 0.07 & 0.09 & 0.15 & 0.35 \\
\hline G II-IV, $-1<[\mathrm{M} / \mathrm{H}]<-0.5 \mathrm{dex}$ & 81 & 110 & 241 & 426 & 0.15 & 0.25 & 0.54 & 0.98 & 0.08 & 0.10 & 0.17 & 0.44 \\
\hline G II-IV, $-2<[\mathrm{M} / \mathrm{H}]<-1 \operatorname{dex}$ & 98 & 164 & 282 & 472 & 0.25 & 0.46 & 0.74 & 1.08 & 0.10 & 0.13 & 0.23 & 0.43 \\
\hline G II-IV, $[\mathrm{M} / \mathrm{H}]<-2 \mathrm{dex}$ & 187 & 248 & 375 & 553 & 0.37 & 0.61 & 0.99 & 1.07 & 0.17 & 0.26 & 0.49 & 0.60 \\
\hline F II-IV all $[\mathrm{M} / \mathrm{H}]$ & 79 & 73 & 138 & 140 & 0.14 & 0.13 & 0.14 & 0.15 & 0.09 & 0.09 & 0.10 & 0.27 \\
\hline $\mathrm{K} \mathrm{V},[\mathrm{M} / \mathrm{H}]>-0.5 \mathrm{dex}$ & 66 & 69 & 92 & 171 & 0.11 & 0.14 & 0.22 & 0.34 & 0.08 & 0.09 & 0.09 & 0.21 \\
\hline $\mathrm{K} \mathrm{V},-1<[\mathrm{M} / \mathrm{H}]<-0$ & 75 & 85 & 112 & 225 & 0.15 & 0.17 & 0.24 & 0.38 & 0.09 & 0.10 & 0.13 & 0.30 \\
\hline $\mathrm{K} \mathrm{V},-2<[\mathrm{M} / \mathrm{H}]<-1$ & 83 & 98 & 173 & 328 & 0.16 & 0.19 & 0.25 & 0.51 & 0.09 & 0.11 & 0.14 & 0.35 \\
\hline $\mathrm{K} \mathrm{V},[\mathrm{M} / \mathrm{H}]<-2 \mathrm{dex}$ & 93 & 133 & 278 & 518 & 0.11 & 0.17 & 0.47 & 1.03 & 0.06 & 0.06 & 0.15 & 0.38 \\
\hline $\mathrm{G} \mathrm{V},[\mathrm{M} / \mathrm{H}]>-0.5 \mathrm{dex}$ & 67 & 98 & 209 & 344 & 0.10 & 0.16 & 0.33 & 0.51 & 0.09 & 0.10 & 0.14 & 0.30 \\
\hline $\mathrm{G} \mathrm{V},-1<[\mathrm{M} / \mathrm{H}]<-0$ & 87 & 147 & 246 & 426 & 0.14 & 0.22 & 0.36 & 0.55 & 0.09 & 0.12 & 0.21 & 0.38 \\
\hline $\mathrm{G} \mathrm{V},-2<[\mathrm{M} / \mathrm{H}]<-1$ & 119 & 181 & 358 & 669 & 0.19 & 0.32 & 0.44 & 0.71 & 0.11 & 0.14 & 0.28 & 0.54 \\
\hline $\mathrm{G} \mathrm{V},[\mathrm{M} / \mathrm{H}]<-2 \mathrm{dex}$ & 279 & 435 & 690 & 843 & 0.44 & 0.54 & 0.72 & 1.06 & 0.26 & 0.38 & 0.61 & 0.80 \\
\hline $\mathrm{F} \mathrm{V},[\mathrm{M} / \mathrm{H}]>-0.5 \mathrm{dex}$ & 81 & 117 & 307 & 493 & 0.13 & 0.18 & 0.34 & 0.52 & 0.11 & 0.13 & 0.27 & 0.43 \\
\hline $\mathrm{F} \mathrm{V},-1<[\mathrm{M} / \mathrm{H}]<-0$ & 96 & 151 & 306 & 575 & 0.14 & 0.21 & 0.33 & 0.49 & 0.11 & 0.14 & 0.26 & 0.48 \\
\hline $\mathrm{F} \mathrm{V},-2<[\mathrm{M} / \mathrm{H}]<-1$ & 155 & 257 & 563 & 999 & 0.21 & 0.30 & 0.40 & 0.69 & 0.13 & 0.19 & 0.43 & 0.83 \\
\hline $\mathrm{F} \mathrm{V},[\mathrm{M} / \mathrm{H}]<-2 \mathrm{dex}$ & 447 & 641 & 1046 & 1165 & 0.43 & 0.65 & 0.84 & 1.22 & 0.40 & 0.53 & 0.95 & 1.14 \\
\hline Thin disk dwarfs & 66 & 89 & 199 & 344 & 0.09 & 0.14 & 0.32 & 0.50 & 0.09 & 0.10 & 0.13 & 0.29 \\
\hline Thick disk dwarfs & 91 & 146 & 280 & 501 & 0.14 & 0.22 & 0.35 & 0.52 & 0.09 & 0.13 & 0.24 & 0.43 \\
\hline Halo giants & 90 & 149 & 244 & 443 & 0.23 & 0.43 & 0.70 & 1.05 & 0.10 & 0.13 & 0.24 & 0.39 \\
\hline
\end{tabular}

Notes. Luminosity classes I and II assume $\log g \leqslant 3.5$, luminosity class $\mathrm{V}$ assumes $\log g>3.5$. Spectral types are defined by $T_{\text {eff }}$ ranges as follows: $T_{\text {eff }}<5000 \mathrm{~K}$ for K-type, $5000 \leqslant T_{\text {eff }}<6000 \mathrm{~K}$ for G-type, and $T_{\text {eff }} \geqslant 6000 \mathrm{~K}$ for F-type stars.

Table 2

Internal Errors after Re-normalizations with Photometric Priors

\begin{tabular}{|c|c|c|c|c|c|c|c|c|c|c|c|c|}
\hline \multirow[t]{2}{*}{$\overline{\mathrm{S} / \mathrm{N}\left(\mathrm{pixel}^{-1}\right)}$} & \multicolumn{4}{|c|}{$T_{\text {eff }}(\mathrm{K})$} & \multicolumn{4}{|c|}{$\log g(\operatorname{dex})$} & \multicolumn{4}{|c|}{$[\mathrm{M} / \mathrm{H}](\mathrm{dex})$} \\
\hline & 100 & 50 & 20 & 10 & 100 & 50 & 20 & 10 & 100 & 50 & 20 & 10 \\
\hline K II-IV, $[\mathrm{M} / \mathrm{H}]>-0.5 \mathrm{dex}$ & 71 & 76 & 112 & 180 & 0.12 & 0.14 & 0.24 & 0.50 & 0.08 & 0.08 & 0.11 & 0.21 \\
\hline K II-IV, $-1<[\mathrm{M} / \mathrm{H}]<-0.5 \mathrm{dex}$ & 61 & 86 & 137 & 285 & 0.14 & 0.20 & 0.40 & 0.69 & 0.08 & 0.10 & 0.17 & 0.29 \\
\hline K II-IV, $-2<[\mathrm{M} / \mathrm{H}]<-1 \mathrm{dex}$ & 75 & 96 & 173 & 312 & 0.20 & 0.31 & 0.59 & 1.02 & 0.09 & 0.11 & 0.21 & 0.34 \\
\hline K II-IV, $[\mathrm{M} / \mathrm{H}]<-2 \operatorname{dex}$ & 75 & 101 & 213 & 399 & 0.27 & 0.35 & 0.79 & 0.98 & 0.10 & 0.10 & 0.18 & 0.32 \\
\hline G II-IV, $[\mathrm{M} / \mathrm{H}]>-0.5 \mathrm{dex}$ & 78 & 104 & 237 & 332 & 0.09 & 0.21 & 0.48 & 0.49 & 0.07 & 0.09 & 0.18 & 0.26 \\
\hline G II-IV, $-1<[\mathrm{M} / \mathrm{H}]<-0.5 \mathrm{dex}$ & 79 & 103 & 238 & 412 & 0.15 & 0.23 & 0.52 & 0.97 & 0.08 & 0.10 & 0.17 & 0.37 \\
\hline G II-IV, $-2<[\mathrm{M} / \mathrm{H}]<-1 \mathrm{dex}$ & 90 & 158 & 283 & 412 & 0.21 & 0.45 & 0.78 & 1.05 & 0.11 & 0.12 & 0.23 & 0.33 \\
\hline G II-IV, $[\mathrm{M} / \mathrm{H}]<-2 \operatorname{dex}$ & 203 & 265 & 378 & 479 & 0.33 & 0.61 & 0.91 & 1.14 & 0.15 & 0.25 & 0.49 & 0.54 \\
\hline F II-IV all $[\mathrm{M} / \mathrm{H}]$ & 79 & 83 & 93 & 138 & 0.14 & 0.13 & 0.14 & 0.26 & 0.09 & 0.09 & 0.09 & 0.31 \\
\hline $\mathrm{K} V,[\mathrm{M} / \mathrm{H}]>-0.5 \mathrm{dex}$ & 66 & 69 & 92 & 168 & 0.11 & 0.15 & 0.22 & 0.36 & 0.08 & 0.09 & 0.09 & 0.20 \\
\hline $\mathrm{K} \mathrm{V},-1<[\mathrm{M} / \mathrm{H}]<-0.5 \mathrm{dex}$ & 75 & 84 & 110 & 219 & 0.15 & 0.17 & 0.23 & 0.38 & 0.09 & 0.10 & 0.12 & 0.29 \\
\hline $\mathrm{K} \mathrm{V},-2<[\mathrm{M} / \mathrm{H}]<-1 \mathrm{dex}$ & 82 & 97 & 172 & 293 & 0.16 & 0.19 & 0.23 & 0.52 & 0.08 & 0.10 & 0.14 & 0.33 \\
\hline $\mathrm{K} \mathrm{V},[\mathrm{M} / \mathrm{H}]<-2 \mathrm{dex}$ & 92 & 144 & 240 & 480 & 0.11 & 0.17 & 0.46 & 0.54 & 0.07 & 0.07 & 0.17 & 0.39 \\
\hline $\mathrm{G} \mathrm{V},[\mathrm{M} / \mathrm{H}]>-0.5 \mathrm{dex}$ & 66 & 95 & 203 & 316 & 0.10 & 0.15 & 0.35 & 0.55 & 0.09 & 0.10 & 0.15 & 0.30 \\
\hline $\mathrm{G} \mathrm{V},-1<[\mathrm{M} / \mathrm{H}]<-0.5 \mathrm{dex}$ & 85 & 144 & 238 & 360 & 0.14 & 0.22 & 0.38 & 0.55 & 0.09 & 0.12 & 0.20 & 0.33 \\
\hline $\mathrm{G} \mathrm{V},-2<[\mathrm{M} / \mathrm{H}]<-1 \mathrm{dex}$ & 101 & 169 & 291 & 441 & 0.15 & 0.28 & 0.43 & 0.62 & 0.10 & 0.13 & 0.24 & 0.39 \\
\hline $\mathrm{G} \mathrm{V},[\mathrm{M} / \mathrm{H}]<-2 \operatorname{dex}$ & 220 & 317 & 393 & 480 & 0.35 & 0.46 & 0.53 & 0.80 & 0.21 & 0.30 & 0.39 & 0.50 \\
\hline $\mathrm{F} \mathrm{V},[\mathrm{M} / \mathrm{H}]>-0.5 \mathrm{dex}$ & 66 & 94 & 257 & 464 & 0.13 & 0.18 & 0.38 & 0.56 & 0.10 & 0.11 & 0.25 & 0.41 \\
\hline $\mathrm{F} \mathrm{V},-1<[\mathrm{M} / \mathrm{H}]<-0.5 \mathrm{dex}$ & 84 & 127 & 277 & 498 & 0.14 & 0.21 & 0.35 & 0.52 & 0.09 & 0.13 & 0.25 & 0.43 \\
\hline $\mathrm{F} \mathrm{V},-2<[\mathrm{M} / \mathrm{H}]<-1 \mathrm{dex}$ & 104 & 182 & 440 & 601 & 0.18 & 0.25 & 0.38 & 0.53 & 0.11 & 0.15 & 0.32 & 0.51 \\
\hline $\mathrm{F} \mathrm{V},[\mathrm{M} / \mathrm{H}]<-2 \mathrm{dex}$ & 331 & 503 & 617 & 678 & 0.32 & 0.51 & 0.54 & 0.66 & 0.33 & 0.48 & 0.54 & 0.69 \\
\hline Thin disk dwarfs & 61 & 85 & 183 & 330 & 0.09 & 0.14 & 0.33 & 0.53 & 0.09 & 0.10 & 0.12 & 0.30 \\
\hline Thick disk dwarfs & 84 & 133 & 256 & 406 & 0.14 & 0.21 & 0.38 & 0.54 & 0.09 & 0.12 & 0.22 & 0.37 \\
\hline Halo giants & 83 & 143 & 258 & 399 & 0.21 & 0.41 & 0.74 & 1.04 & 0.10 & 0.12 & 0.24 & 0.38 \\
\hline
\end{tabular}


in the case of spectral degeneracy, the associated errors are larger, being consistent with what is expected.

\subsection{Description of the Observed Input Spectra}

The extraction and reduction procedures applied to the observed spectra are as described in Steinmetz et al. (2006) and Zwitter et al. (2008). As far as the normalization and restframe corrections are concerned, we have used the results and the raw data coming from DR3, details of which can be found in Siebert et al. (2011b) and for which the general properties are summarized in Section 8. In addition, in order to be able to perform a pattern recognition and a pixel-to-pixel comparison with the spectra of the learning grid, the RAVE spectra have been interpolated at the wavelengths of the templates and the cores of the IR Ca II triplet lines have been removed (see Section 3.4).

Since the DR4 pipeline re-normalizes the observed spectra, initially erroneous or inaccurate continuum shapes do not influence the final parameter accuracy of DR4. One might worry about the accuracy of the RV of the stars and hence their rest-frame correction. Indeed, the radial velocities have been computed using cross-correlation with the solution template derived in DR3, whereas in the present work, the atmospheric parameters are recomputed, leading to different values. Nevertheless, the pipeline requires an RV precision only better than $\sim 7-10 \mathrm{~km} \mathrm{~s}^{-1}$ (see K11) in order not to be affected by Doppler shifts in the parameter estimation. This threshold is much higher than the accuracy of the radial velocities coming from the DR3, where $95 \%$ of the sample has errors of less than $\sim 4 \mathrm{~km} \mathrm{~s}^{-1}$ and $98 \%$ less than $7 \mathrm{~km} \mathrm{~s}^{-1}$ (Siebert et al. 2011b, and Section 8). Thus, we can assume that the spectra are indeed at the rest frame for the purpose of our analysis.

\section{VALIDATION OF THE PARAMETERIZATION WITH EXTERNAL DATA SETS}

Up to here in this paper, only the internal performances of the pipeline have been discussed. Nevertheless, any given pipeline based on a grid of synthetic spectra needs to be verified and calibrated on observed spectra with high $\mathrm{S} / \mathrm{N}$ and welldetermined parameters. The grid of synthetic spectra that has been used for this work has been computed with an atomic line list calibrated on the high-resolution and high-S/N spectra of the Sun and Arcturus of Brault \& Neckel (1987) and Hinkle et al. (2003). However, these calibrations concern only two particular stars, and further investigation needs to be done in order to correct possible biases in the parameter's estimation. In order to calibrate the $T_{\text {eff }}, \log g$, and $[\mathrm{M} / \mathrm{H}]$, instead of going through the process of calibrating all the lines for many reference stars, and improve the quality of the atmosphere modeling, one can also validate the pipeline's parameter results with reference parameter measurements from the literature.

This calibrating data set needs to cover as much as possible the parameter space investigated by the survey. Ideally the calibration of the parameters would be done using only RAVE spectra of suitable standards, but RAVE-like spectra, at the same resolution and (if possible) reduced in the same manner, can be sufficient in the case where not enough calibration spectra are available.

\subsection{The Calibration Data Sets of Observed Spectra}

First, the RAVE database has been explored to find spectra of stars that had atmospheric parameter determinations available from high-resolution spectroscopy. For that purpose, we made extensive use of the heterogeneous PASTEL catalog ${ }^{30}$ to identify such targets, retrieving roughly 400 star candidates. Following Soubiran et al. (2010), we considered only the reference values coming from Fuhrmann (1998a, 1998b, 2004, 2008), Gratton et al. (1996, 2003), Hekker \& Meléndez (2007), Luck \& Heiter (2006, 2007), McWilliam (1990), Mishenina \& Kovtyukh (2001), Mishenina et al. (2004, 2006, 2008), Ramírez et al. (2007), and Valenti \& Fischer (2005). These studies, when considered by author, all include a large number of stars (at minimum 222 stars) and are all analyzed in a homogeneous way. This allows us to minimize the discrepancies between the sub-catalogs of PASTEL. When for a given star several measurements were available, the mean was computed and the dispersion of the parameters has been considered as the uncertainty on the reference values. We kept only those stars for which measurements were available for the three parameters from a single study, and for which the dispersions among the literature values were less than $100 \mathrm{~K}, 0.2 \mathrm{dex}$, and $0.1 \mathrm{dex}$ for $T_{\text {eff }}, \log g$, and $[\mathrm{M} / \mathrm{H}]$, respectively. In total, 169 stars were selected that way, mainly dwarfs of intermediate metallicity.

In order to investigate the pipeline's behavior in the lowmetallicity regime for giant stars, we chose to use the parameters of 229 thick disk stars analyzed by Ruchti et al. (2011), as well as 163 stars observed by J. P. Fulbright et al. (in preparation). The targets of both of these data sets are drawn from RAVE, while the stellar parameters have been obtained from an equivalent-width (EW) analysis of high-resolution spectra. In addition, the very metal-poor giant star CD-38245 $([\mathrm{M} / \mathrm{H}]=-4.2$ dex; Cayrel et al. 2004), which has been observed twice by RAVE, has been included in the list, in order to calibrate the results at the very metal-poor regime.

Metal-rich giant stars have been explored thanks to the CFLIB library (Valdes et al. 2004). The entire spectral library was downloaded from the Web site ${ }^{31}$ of that project, excluding only spectra that did not include the wavelength range around the IR Ca II triplet. The final comparison catalog is the same as in $\mathrm{K} 11$, where once again we used the updated values that can be found in the PASTEL database and discarded the stars for which the dispersions in the literature values were greater than $100 \mathrm{~K}$, 0.2 dex, and 0.1 dex for $T_{\text {eff }}, \log g$, and $[\mathrm{M} / \mathrm{H}]$, respectively.

Finally, in order to have a more significant statistical sample at the high-metallicity regime, we used the $2.3 \mathrm{~m}$ telescope at the Siding Spring Observatory (SSO) to obtain spectra of stars belonging to open clusters. Although the data have not been obtained with the same instrument, the same reduction pipeline has been used as for the RAVE spectra. For calibration purposes, we have used 16 RAVE-like SSO spectra of giant stars belonging to the open cluster M67 and 12 RAVE-like SSO spectra of giants belonging to the open cluster IC 4651, with a few additional data sets used as testing sets (see Section 4.5). These targets were selected given their positions, colors, and radial velocities when available, prioritizing bright stars in order to have high-S/N spectra. For these stars, no individual atmospheric parameters were available, but their metallicity is expected to have a small dispersion around their mean open cluster metallicity value.

In total, 809 stars are used as calibrators, each having $\mathrm{S} / \mathrm{N}>40 \mathrm{pixel}^{-1}$. The final number of spectra used from each data set is summarized in Table 3, and their reference and retrieved $T_{\text {eff }}-\log g$ diagrams are plotted in Figure 5.

\footnotetext{
30 http://pastel.obs.u-bordeaux 1.fr/

$31 \mathrm{http} / / /$ www.noao.edu/cflib/
} 
Table 3

Calibration Data Sets with $\mathrm{S} / \mathrm{N}>40 \mathrm{pixel}^{-1}$

\begin{tabular}{|c|c|c|c|c|}
\hline Data Set & Object & N Spectra & {$[\mathrm{Fe} / \mathrm{H}]$} & Reference for the Stellar Parameters \\
\hline IC 4651 & Open cluster & 6 & +0.10 & Pasquini et al. (2004) \\
\hline M67 & Open cluster & 16 & +0.05 & Pancino et al. (2010) \\
\hline CFLIB & Dwarfs and giants & 224 & {$[-1.0,0.0]$} & PASTEL database \\
\hline CD-38245 & $T_{\text {eff }}=4800 \mathrm{~K}, \log g=1.5$ & 2 & -4.2 & Cayrel et al. (2004) \\
\hline Ruchti et al. (2011) ${ }^{\mathrm{a}}$ & Giants and dwarfs & 229 & {$[-2.5 ;-0.5]$} & Ruchti et al. (2011) \\
\hline Fulbright et al. & Giants & 163 & {$[-2.5 ; 0.0]$} & J. P. Fulbright et al. (in preparation) \\
\hline RAVE spectra & Giants and dwarfs & 169 & {$[-1.5 ; 0.0]$} & PASTEL database \\
\hline
\end{tabular}

Note. ${ }^{a}$ For the Ruchti et al. (2011) catalog we selected stars with $[\mathrm{M} / \mathrm{H}]>-2.5$ dex and $\log g<3$ and stars with $[\mathrm{M} / \mathrm{H}]<-0.8$ dex and $\log g>3$.
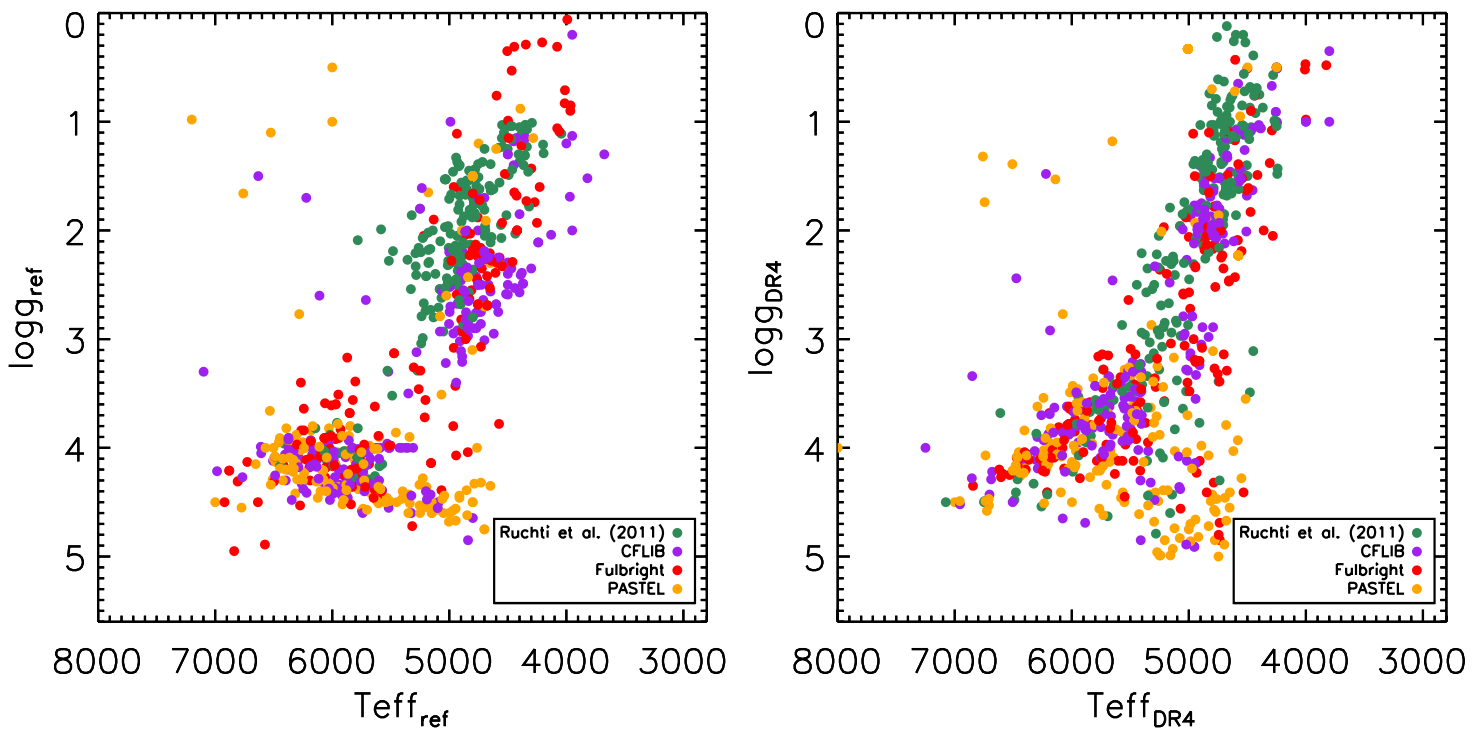

Figure 5. Surface gravity $(\log g)$ vs. effective temperature $\left(T_{\text {eff }}\right)$ diagram of the calibration data sets for which we have parameter estimations coming from highresolution spectroscopy. On the left-hand side are represented the values found in the literature, whereas on the right-hand side are plotted the results obtained from the DR4 pipeline.

(A color version of this figure is available in the online journal.)
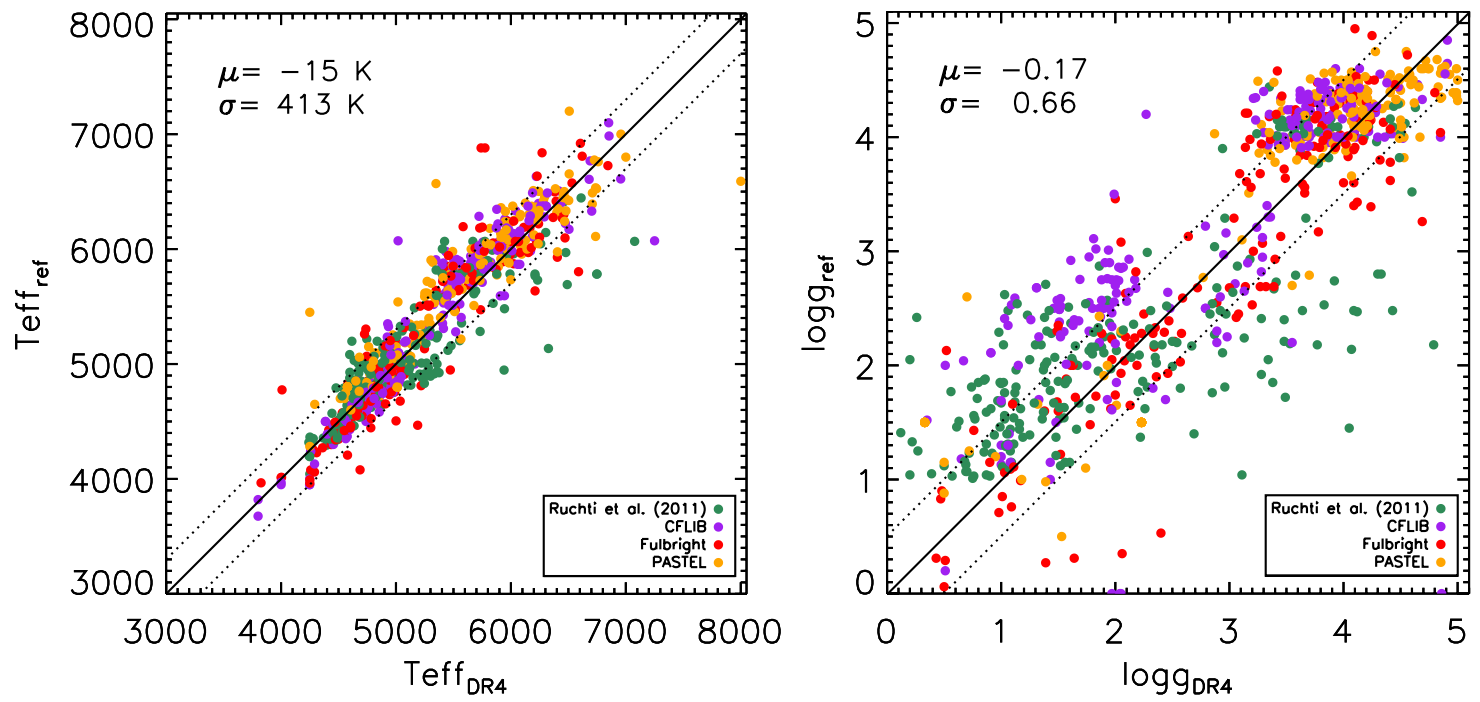

Figure 6. Comparison of the reference values found in the literature and the derived effective temperatures (left panel) and surface gravities (right panel). Color-coding for each data set is the same as in Figure 5. Dotted diagonal lines represent offsets from unity of $\pm 300 \mathrm{~K}$ and \pm 0.5 dex for $T_{\text {eff }}$ and log $g$, respectively. The mean offsets $(\mu)$ and the dispersions $(\sigma)$ are indicated in the upper left corner of each plot.

(A color version of this figure is available in the online journal.)

\subsection{Validation of the Effective Temperatures and Surface Gravities}

Figures 5 and 6 show the comparison between the reference values found in the literature and those found with the present pipeline for all the data sets except those for open cluster members (where no reference values were available). As far as the effective temperature is concerned, good agreement is found, with a mean offset of $15 \mathrm{~K}$ and a dispersion of roughly $400 \mathrm{~K}$. 


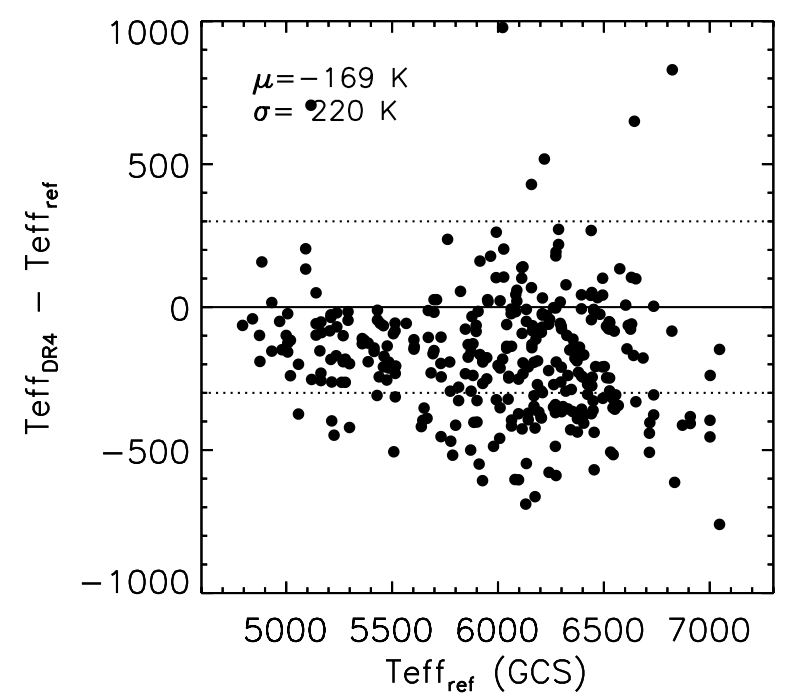

Figure 7. Comparison of the effective temperatures found by the DR4 pipeline for RAVE spectra of stars that are part of the Geneva-Copenhagen Survey with the updated values of Casagrande et al. (2011). The constant offset of $\sim 170 \mathrm{~K}$ shows that DR4 is not on the same effective temperature scale.

On the other hand, the agreement is less good for the surface gravity, with a rather big scatter for the giant stars. This effect is a manifestation of the previously cited spectral degeneracy that is present for the low- and intermediate-resolution spectra around the IR Ca II triplet. According to the stochastic position of the noise on the spectrum, a metal-rich turn-off star can be easily confused with a star on the sub-giant branch of lower metallicity. Unless precise photometric temperatures are known, this degeneracy cannot be lifted using medium-resolution spectra alone and is a true degeneracy. Nevertheless, as noted in the previous sections, the DR4 pipeline takes advantage of the 2MASS photometric information (see Section 3.4), hence partly reducing the effect of these degeneracies.

Discussion on the effective temperature scale. In order to verify the determination of the effective temperatures, we compared the DR4 values of RAVE spectra with $\mathrm{S} / \mathrm{N}>20 \mathrm{pixel}^{-1}$ for
327 stars in common with the photometric effective temperatures from the Casagrande et al. (2010) calibration of the Geneva-Copenhagen Survey (GCS; Nordström et al. 2004). Figure 7 shows on one hand a small dispersion of the DR4 pipeline's effective temperatures when comparing with the values published by Casagrande et al. (2011) for the GCS, but on the other hand there is a constant underestimation of $\sim 170 \mathrm{~K}$. Nevertheless, since the GCS covers only a limited range of the parameter space (only metal-rich dwarfs), and because any such offset is not seen with the other calibration data sets, it has been decided not to apply any correction to the RAVE $T_{\text {eff }}$ scale. We note though that the user of the DR4 effective temperatures should be aware that in order to be in agreement with the Casagrande et al. (2010) effective temperature scale, for the type of stars analyzed by the GCS, an offset correction should be performed.

\subsection{Overall Metallicity Calibration}

In order to investigate the calibration needs for the metallicities, we used the iron abundances $([\mathrm{Fe} / \mathrm{H}])$ from the literature. Indeed, we recall that the iron-peak and the $\alpha$-element abundances of the synthetic spectra are scaled to the iron abundance. Since the metallicity measurement is dominated by the Ca II lines (which correspond to an $\alpha$-element), in our case and for standard Galactic $\alpha$-abundances (i.e., following the trend defined in Section 3.4) we have $[\mathrm{M} / \mathrm{H}]_{\mathrm{DR} 4} \approx[\mathrm{Fe} / \mathrm{H}]$. Nevertheless, for non-standard stars, the overall metallicity will not be equal to the iron abundance, and hence it has been decided to keep in what follows the notation $[\mathrm{M} / \mathrm{H}]$ instead of $[\mathrm{Fe} / \mathrm{H}]$. In what follows, the nomenclature of the DR3 paper is adopted, denoting the raw metallicity estimation of the DR4 pipeline as $[\mathrm{m} / \mathrm{H}]$ and the calibrated (final) metallicity as $[\mathrm{M} / \mathrm{H}]$. Comparison of the reference values and those derived by the DR4 pipeline is presented in the left panel of Figure 8.

The results of Figure 8 have been obtained with all the data sets of Table 3, assuming the metallicities given in the fourth column of that table. From the left panel of Figure 8 one can notice that there is an offset between the derived metallicities from the RAVE spectra and the reference iron abundances.
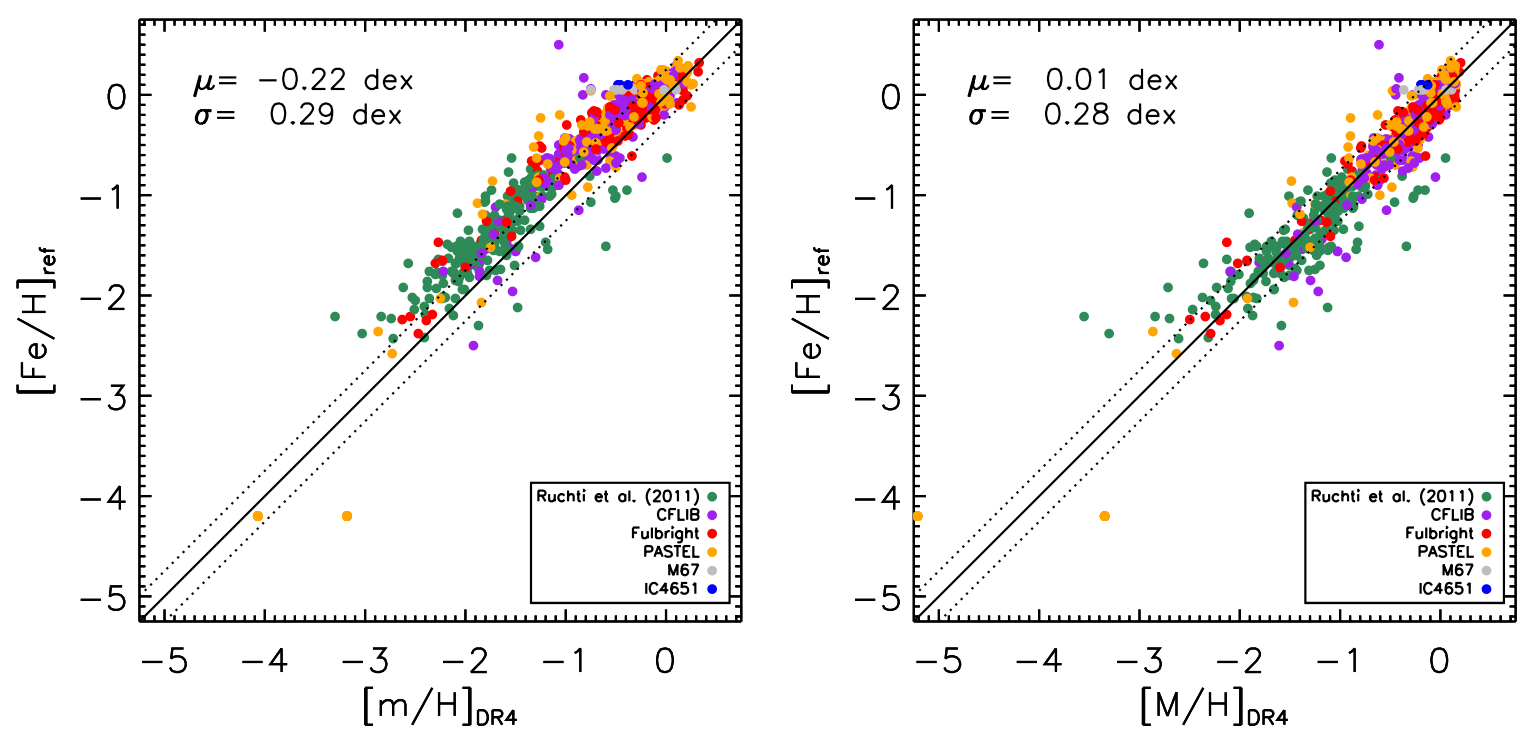

Figure 8. Comparison between the reference iron abundances found in the literature $\left([\mathrm{Fe} / \mathrm{H}]_{\mathrm{ref}}\right)$ and the derived overall metallicities $\left([\mathrm{m} / \mathrm{H}]_{\mathrm{DR} 4}\right.$, left panel) and the calibrated overall metallicities $([\mathrm{M} / \mathrm{H}] \mathrm{DR} 4$, right panel), according to Equation (7). Color-coding for each data set is the same as in Figure 5 . Dotted diagonal lines represent offsets from unity of \pm 0.25 dex.

(A color version of this figure is available in the online journal.) 

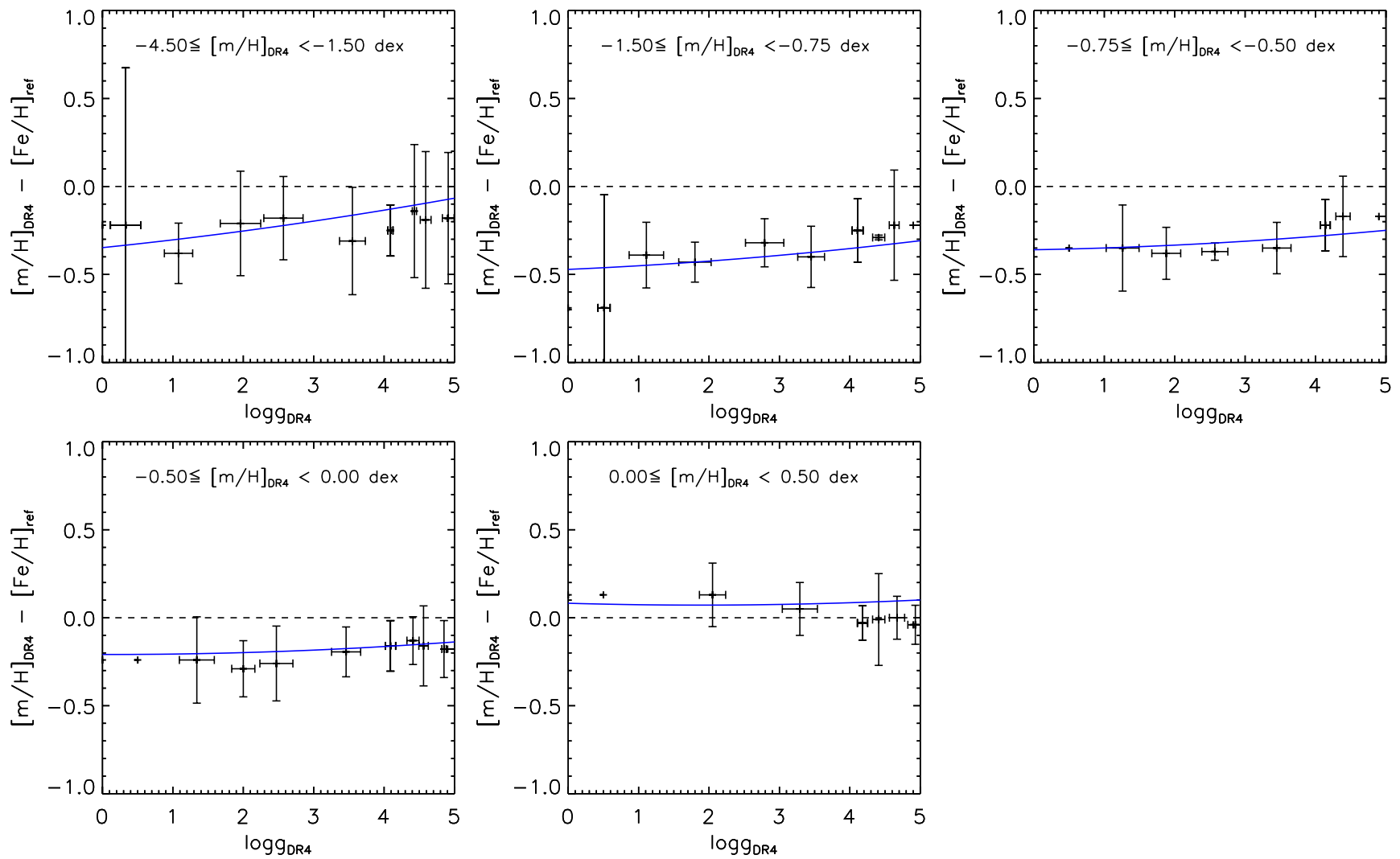

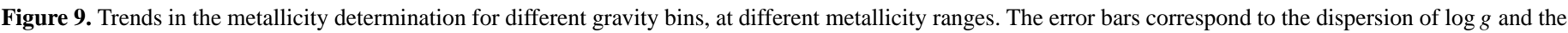

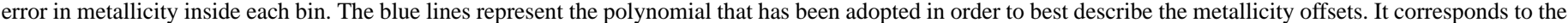
polynomial of Equation (7) and has been computed for the mean metallicity of each subsample.

(A color version of this figure is available in the online journal.)

This bias is not the same for all metallicities, and it is more important for metal-poor stars than in the metal-rich regime. We investigated the correlations of the errors and found that the main parameters driving the bias are the surface gravity and the metallicity itself. Figure 9 illustrates the covariance of the residual errors on the metallicity with respect to the surface gravity, for different metallicity ranges. In this figure, each point and error bar represent the median and the dispersion of the metallicity error for the stars inside each gravity bin. This binning approach smooths the errors, minimizes the impact of outliers, and highlights the general trends of the biases. On one hand, the results of Figure 9 show for the lowest metallicities a rather constant underestimation of the metallicity by 0.2 dex. On the other hand, there are some clear trends in the more metal-rich regimes, where the giant stars exhibit higher offsets than the dwarfs. These trends are too strong to be explained by a variation of microturbulent velocity along the giant branch, where the expected offsets should be less than 0.1 dex (see, e.g., Kirby et al. 2009).

Using the binned points of Figure 9, the resulting fit of a quadratic surface for the errors in metallicity, taking into account the dependences on both the surface gravity and the metallicity, is

$$
\begin{aligned}
{[\mathrm{m} / \mathrm{H}]-[\mathrm{M} / \mathrm{H}]_{\mathrm{ref}}=} & -0.076-0.006 \times \log g+0.003 \\
& \times \log ^{2} g-0.021 \times[\mathrm{m} / \mathrm{H}] \times \log g \\
& +0.582 \times[\mathrm{m} / \mathrm{H}]+0.205 \times[\mathrm{m} / \mathrm{H}]^{2} .
\end{aligned}
$$

Given this relation, the trend for the typical mean metallicity inside each box of Figure 9 has been plotted in blue. As expected, the fits are in good agreement with the offsets, hence assimilating the metallicity calibration relation to Equation (7). The right panel of Figure 8 shows the improvement that has been made on the metallicity determination thanks to the correction of Equation (7). We note, however, that due to the lack of reference stars with super-solar metallicities, our calibration is not optimal for $[\mathrm{M} / \mathrm{H}]>+0.1 \mathrm{dex}$. This limitation will be addressed in a future study.

A more detailed investigation of the residuals for different gravity regimes and with respect to the calibrated metallicity is shown in Figure 10, for the Ruchti, Fulbright, PASTEL, and CFLIB libraries. As expected, there is no bias for the calibrated metallicity (lower plots), nor for the other parameters, except for the surface gravity of the lowest gravity giant stars (see also Section 6.1). The self-consistency of the calibration is hence validated.

\subsection{A Comment on RAVE DRI, DR2, and DR3 Parameters}

Previous RAVE data releases used the Munari et al. (2005) grid of synthetic spectra and a penalized $\chi^{2}$ algorithm in order to determine the effective temperatures, surface gravities, overall metallicities, and $\alpha$-abundances. The stellar rotational velocities $\left(V_{\text {rot }}\right)$ and the microturbulent velocities $(\xi)$ were also left as free parameters, although without attributing any constraint on these values in the end. ${ }^{32}$ Furthermore, the 2MASS photometric information was not used to help reduce spectral degeneracies, and the calibration data sets were not fully available.

\footnotetext{
${ }^{32}$ For DR3 the microturbulent parameter was fixed at $\xi=2 \mathrm{~km} \mathrm{~s}^{-1}$.
} 


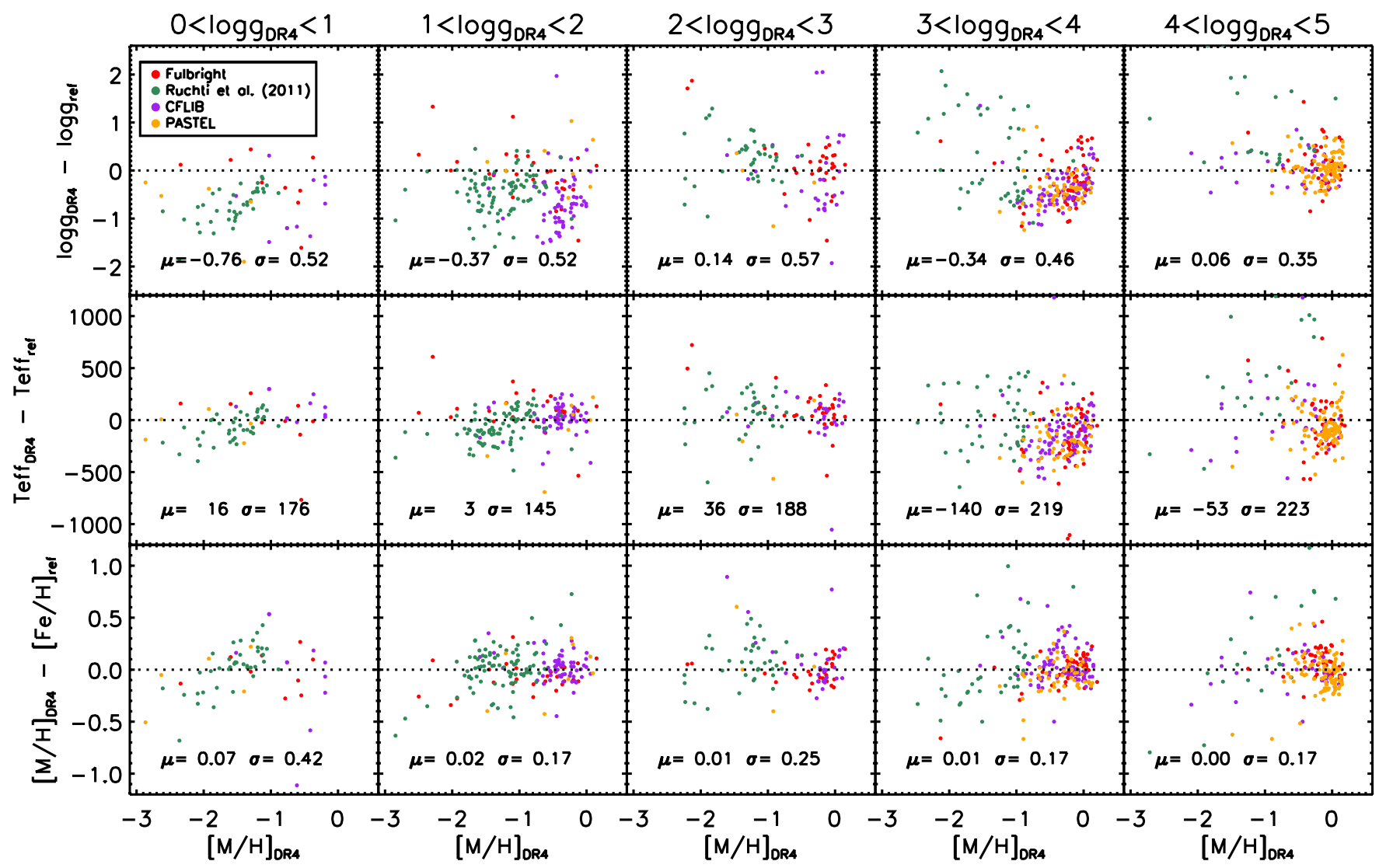

Figure 10. DR4 residual plots for RAVE (RAVE-PASTEL in yellow, Ruchti in green, and Fulbright in red) and RAVE-like spectra (CFLIB, in purple). The trends in the stellar parameter systematics are shown with respect to the calibrated metallicity, divided into different (DR4) $\log g$ bins of 1 dex. In each panel, the median offset and the dispersion is indicated.

(A color version of this figure is available in the online journal.)

The present DR4 pipeline reduces the parameter space to only the three free atmospheric parameters we are trying to measure. In addition to imposing a photometric effective temperature range, the new pipeline explores more efficiently the parameter space, thanks to the decision-tree algorithm. This makes the new results more robust and less susceptible to biases caused by spectral degeneracies.

Efficient exploration of the low-dimension parameter space is crucial for the accurate determination of the atmospheric parameters and calibration of the results. Indeed, tests done on the above-mentioned calibration data sets, using the DR3 pipeline output, showed that the metallicity biases could not be calibrated adequately, especially for the turn-off stars, where the degeneracy of the distance function is the most important (see Figure 11). As will be shown in Section 6.3, this led to biases and interdependences between the DR3 parameters and motivated the effort to develop the approach implemented here in DR4.

\subsection{Sanity Check of the Metallicity Calibration on a Set of Observed Spectra}

Our proposed metallicity calibration relation (see Equation (7)) has been further verified on spectra that are not part of the calibration process. For that purpose, we used the 327 RAVE spectra of the GCS stars described previously, 105 non-RAVE spectra from the $S^{4} N$ library degraded to RAVE resolution (Allende Prieto et al. 2004), and 65 RAVE-like spectra of open and globular cluster stars obtained by the $2.3 \mathrm{~m}$ telescope at the SSO, listed in Table 4. We note though that the reference metallicity values that have been adopted for these test spectra are not as reliable as the calibration data sets. Indeed, except for the $S^{4} N$ library, all the other data sets do not have individual spectroscopically measured metallicities. In addition, non-member stars might be included in the cluster data sets. Finally, the mean metallicity value has been considered for the stars belonging to the globular clusters, whereas dispersions up to few tenths of a dex (Gratton et al. 2004) can be expected in some cases.

The three plots of Figure 12 show the recovered $T_{\text {eff }}-\log g$ diagram of the total considered sample (left), the $\log g$ versus residuals in $[\mathrm{m} / \mathrm{H}]$ (middle), and versus residuals in calibrated $[\mathrm{M} / \mathrm{H}]$ (right). Despite the relatively large dispersion due to the heterogeneous quality of the data sets, one can see that the bias is greatly reduced in all the samples, and for all gravities, providing a sanity validation check of the calibration relation established previously.

\subsection{Computation of the Total Uncertainties of the Pipeline}

The errors described in Section 3.5 concern only the internal accuracies of the method. To estimate the total uncertainties of the pipeline, one needs also to estimate the external errors.

We used all the spectra with $\mathrm{S} / \mathrm{N} \geqslant 50 \mathrm{pixel}^{-1}$ of the previously described calibration data set to estimate the external uncertainties for different ranges of stellar parameters. Given the total number of spectra in the data set, we divided the sample into cool $\left(T_{\text {eff }} \leqslant 6000 \mathrm{~K}\right)$ and hot $\left(T_{\text {eff }}>6000 \mathrm{~K}\right)$ dwarfs $(\log g \geqslant 3.5 \mathrm{dex})$ and giants $(\log g<3.5 \mathrm{dex})$. Furthermore, we also divided into metal-rich $([\mathrm{M} / \mathrm{H}] \geqslant-0.5 \mathrm{dex})$ and metalpoor $([\mathrm{M} / \mathrm{H}]<-0.5 \mathrm{dex})$ regimes, except for the hot giants, for which not enough stars were available in the sample. The 


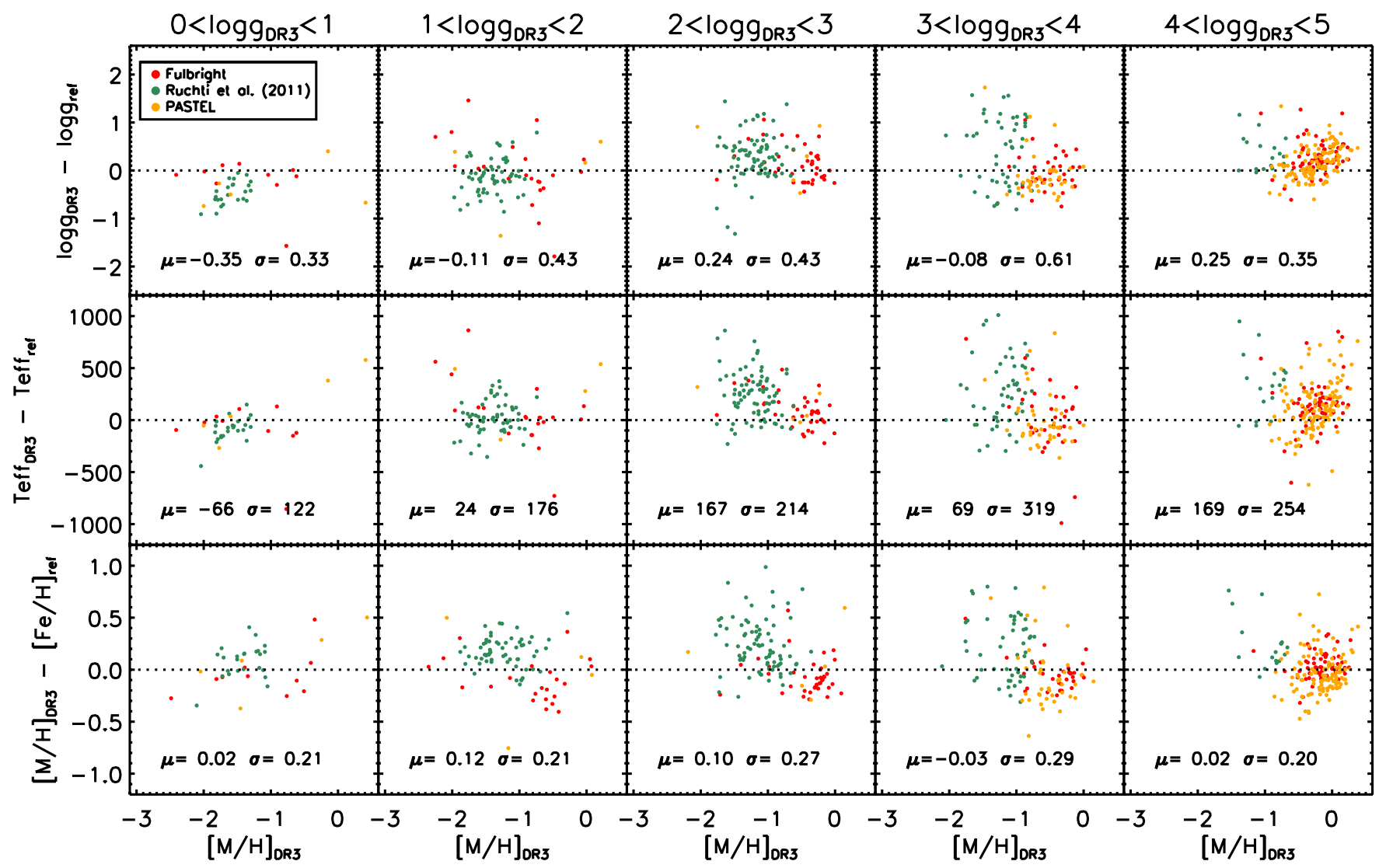

Figure 11. Same as Figure 10 but for the RAVE DR3 pipeline and without the CFLIB analysis. The calibrated metallicities correspond to those obtained using Equation (2) of Siebert et al. (2011b) with parameters $c_{0}=0.578, c_{1}=1.095, c_{3}=1.246, c_{4}=-0.520$. The metallicity trends found for the turn-off stars are representative of the uncalibratable biases present in RAVE DR3.

(A color version of this figure is available in the online journal.)

Table 4

Post-calibration Verification Data Sets

\begin{tabular}{|c|c|c|c|c|c|}
\hline Data Set & Type & $N$ Stars & $\langle[\mathrm{Fe} / \mathrm{H}]\rangle$ & $\sigma([\mathrm{Fe} / \mathrm{H}])$ & Reference \\
\hline M5 & Globular cluster & 8 & -1.28 & 0.11 & Ramírez \& Cohen (2003) \\
\hline NGC 3680 & Open cluster & 7 & -0.04 & 0.03 & Pace et al. (2008) \\
\hline IC4651 & Open cluster & 5 & +0.10 & 0.05 & Pasquini et al. (2004) \\
\hline M67 & Open cluster & 10 & +0.05 & 0.04 & Pancino et al. (2010) \\
\hline NGC 6752 & Globular cluster & 12 & -1.42 & 0.10 & Gratton et al. (2001) \\
\hline NGC 2808 & Globular cluster & 10 & -1.14 & 0.06 & Carretta et al. (2004) \\
\hline NGC 6397 & Globular cluster & 11 & -2.10 & 0.05 & Koch \& McWilliam (2011) \\
\hline Praesepe & Open cluster & 35 & +0.14 & 0.04 & Mean literature value \\
\hline GCS & MW dwarf stars & 327 & solar & $\ldots$ & Casagrande et al. (2011) \\
\hline$S^{4} N$ & MW dwarf stars & 105 & solar & $\ldots$ & Allende Prieto et al. (2004) \\
\hline
\end{tabular}

dispersion of the residual differences is presented in Table 5, together with the number of stars that have been considered in order to compute these uncertainties.

Using the values presented in Table 5, the total uncertainties of the pipeline parameter determinations are then estimated by adding in quadrature the external errors with the internal errors given in Tables 1 and 2.

\section{COMPUTATION OF THE CHEMICAL ABUNDANCES}

The atmospheric parameters inferred in the previous sections are used as an input in order to determine abundances of individual elements. For that purpose, we use an improved version of the RAVE chemical pipeline described in detail in Boeche et al. (2011, afterward B11). Below, we recall the general features of that pipeline and present the current improvements.
The chemical pipeline relies on an EW library that contains the expected EWs of the lines visible in the RAVE wavelength range (604 atomic and molecule lines). These EWs are computed for a grid of stellar parameter values covering the range $[4000,7000] \mathrm{K}$ in $T_{\text {eff }},[0.0,5.0]$ dex in $\log g$, and $[-2.5,+0.5]$ dex in $[\mathrm{M} / \mathrm{H}]$ and five levels of abundances in the range $[-0.4,+0.4]$ dex relative to the metallicity, in steps of 0.2 dex (adopting the solar abundances of Grevesse \& Sauval 1998). The chemical pipeline constructs on-the-fly spectrum models by adopting the effective temperatures and surface gravities obtained by the DR4 pipeline (see Section 3). It then searches for the best-fitting model by minimizing the $\chi^{2}$ between the models and the observations.

For a given normalized, RV-corrected, and wavelengthcalibrated spectrum, the chemical pipeline determines the elemental abundances, following the steps described below: 

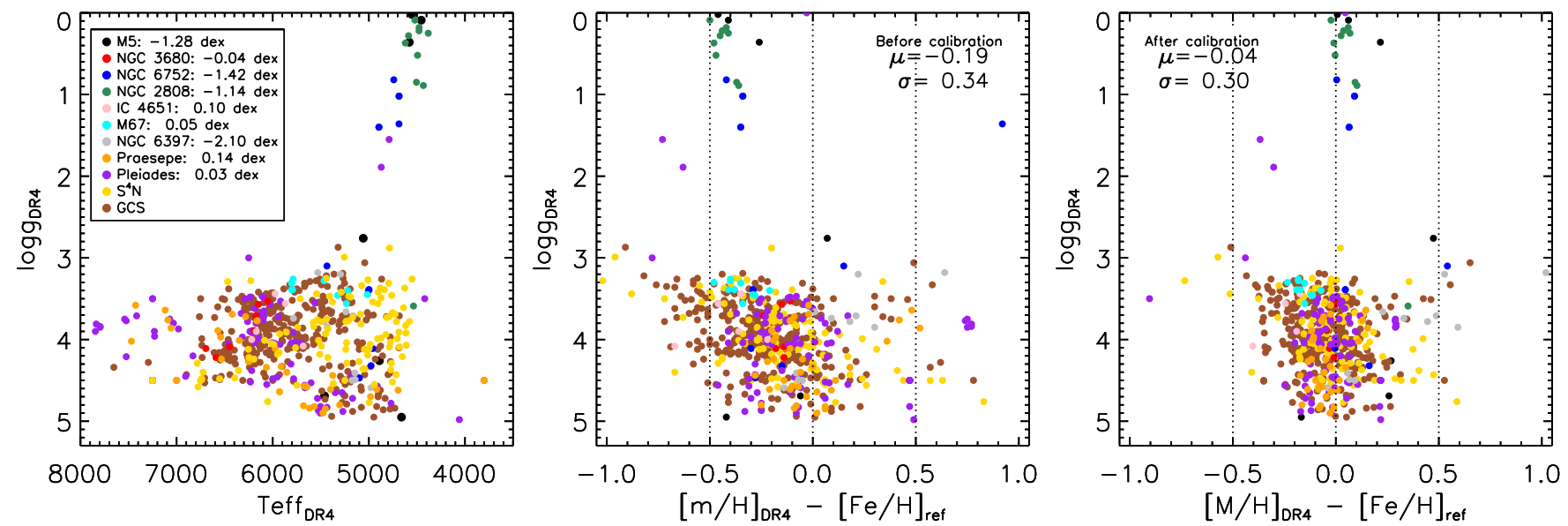

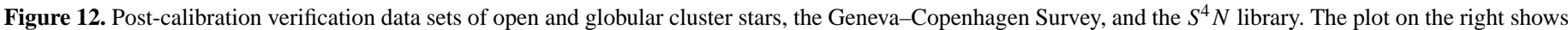

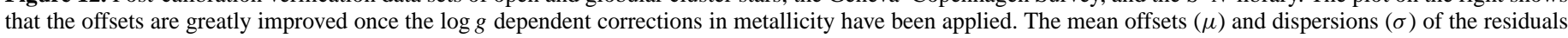
are noted in the upper corner of each plot.

(A color version of this figure is available in the online journal.)

Table 5

Estimation of the External Uncertainties

\begin{tabular}{lrccc}
\hline \hline \multicolumn{5}{c}{ Dwarfs } \\
\hline Parameter Range & \multicolumn{1}{c}{$N$} & $\sigma\left(T_{\text {eff }}\right)$ & $\sigma(\log g)$ & $\sigma([\mathrm{M} / \mathrm{H}])$ \\
\hline$T_{\text {eff }}>6000,[\mathrm{M} / \mathrm{H}]<-0.5$ & 28 & 314 & 0.466 & 0.269 \\
$T_{\text {eff }}>6000,[\mathrm{M} / \mathrm{H}] \geqslant-0.5$ & 104 & 173 & 0.276 & 0.119 \\
$T_{\text {eff }} \leqslant 6000,[\mathrm{M} / \mathrm{H}]<-0.5$ & 97 & 253 & 0.470 & 0.197 \\
$T_{\text {eff }} \leqslant 6000,[\mathrm{M} / \mathrm{H}] \geqslant-0.5$ & 138 & 145 & 0.384 & 0.111 \\
\hline \multicolumn{5}{c}{ Giants } \\
\hline Parameter Range & $N$ & $\sigma\left(T_{\text {eff }}\right)$ & $\sigma(\log g)$ & $\sigma([\mathrm{M} / \mathrm{H}])$ \\
\hline$T_{\text {eff }}>6000$ & 8 & 263 & 0.423 & 0.300 \\
$T_{\text {eff }} \leqslant 6000,[\mathrm{M} / \mathrm{H}]<-0.5$ & 273 & 191 & 0.725 & 0.217 \\
$T_{\text {eff }} \leqslant 6000,[\mathrm{M} / \mathrm{H}] \geqslant-0.5$ & 136 & 89 & 0.605 & 0.144 \\
\hline
\end{tabular}

1. Upload the EWs for the lines at the estimated DR4 $T_{\text {eff }}, \log g,[\mathrm{M} / \mathrm{H}]$, and for the five different abundance levels.

2. Keep only the lines that, at the given stellar parameters, have large enough EWs to be visible above the noise. Mathematically, the condition to satisfy is the following:

$$
\mathrm{EW}(\mathrm{m} \AA)>\frac{\sqrt{2 \pi} \sigma_{\mathrm{res}}}{\mathrm{S} / \mathrm{N}} \cdot 1000,
$$

where $\sigma_{\text {res }}=0.56 \AA$ is the standard deviation of the RAVE Gaussian line profile. In practice, any absorption line whose intensity is larger than $1 \sigma$ of the noise fulfills this condition.

3. Fit the strong $\mathrm{Ca}$ II and $\mathrm{HI}$ lines and correct the continuum (see Section 5.3).

4. Construct the curve of growth (COG) of the lines by fitting a polynomial function through the five $\mathrm{EW}$-abundance points.

5. Create the model by assuming a Voigt profile for each line and summing these profiles together (see Section 5.2).

6. Vary the chemical elemental abundances to obtain different models by changing the EWs of the lines according to their COG.

7. Finally, minimize the $\chi^{2}$ between the models and the observed spectrum to find the best-matching model.
Further details on the line list and the way the EW library has been constructed can be found in B11. In the following subsections we describe the changes that have been brought to B11. These concern a better consideration of the opacity of neighboring lines, an implementation of a pseudoVoigt profile to model the lines, and an improved continuum re-normalization.

\subsection{Equivalent-width Corrections for the Opacity of the Neighboring Lines}

The EW library is built using the driver ewfind of the spectrum synthesis code MOOG (Sneden 1973), which computes the EW of every line as if they were isolated. Nevertheless, line blends when not carefully taken into account can lead to abundance overestimations. In the case of lines instrumentally (but not physically) blended, the observed blend has a total EW that is the sum of the EWs of the two isolated lines, and thus no problem arises. However, when two lines are physically blended (i.e., not instrumentally), the quantity of radiation absorbed by one line is affected by the opacity of the neighboring line, and the total EW of the blend is smaller than the sum of the two isolated EWs. In this case the blend in the constructed model is too strong, leading to abundance overestimations. In order to avoid such overestimation, we corrected the EWs of the blended lines in the EW library with the following procedure:

1. Consider the line $l_{0}$ having $\mathrm{EW}_{0}$, blended with some lines $l_{i}$ with $\mathrm{EW}_{i}$. Compute the ratio $\mathrm{EW}_{r}=\mathrm{EW}_{0} / \sum \mathrm{EW}_{i}$ with $\mathrm{EW}_{0}$ and $\mathrm{EW}_{i}$ computed as if they were isolated.

2. Synthesize the blend composed by $l_{0}$ and all $l_{i}$, and measure the overall $\mathrm{EW}_{\text {tot }}$.

3. Compute the corrected $\mathrm{EW}$ of the line $l_{0}$ as $\mathrm{EW}_{0}^{\text {corr }}=$ $\mathrm{EW}_{r} \cdot \mathrm{EW}_{\text {tot }}$.

Two lines are considered blended if they are closer than $0.2 \AA$. In addition, we applied this correction to lines that are blended with one or more lines having EW > $10 \mathrm{~m} \AA$. Lines with EWs smaller than $10 \mathrm{~m} \AA$ would affect the EW of the neighboring lines by less than $0.7 \%$, which can be considered negligible. Although $\mathrm{EW}_{0}^{\text {corr }}$ is only an approximation, the constructed blends with such corrected EWs match the synthesized blend better than $1 \%$ of the normalized flux. This correction replaces the previous one adopted in the B11 chemical pipeline. 


\subsection{Improved Line Profile}

Most of the absorption lines in the RAVE wavelength range and resolution have an intrinsic width smaller than the RAVE instrumental profile. Therefore, their line profile is dominated by the instrumental one, which is Gaussian. Nevertheless, this is not the case for the strongest lines, where the broad wings extend beyond the instrumental profile. In that case, the line is better approximated by a Voigt profile.

Compared to B11, the new chemical pipeline drops the simplistic Gaussian assumption and now uses an improved line profile. Because of the difficulties of implementing a real Voigt profile, we use the approximation implemented by Bruce et al. (2000):

$$
V(x)=\mathrm{EW} \cdot[r L(x)+(1-r) G(x)],
$$

where $L$ and $G$ are the Lorentzian and Gaussian functions, respectively, and EW is expressed in $\AA$. The $r$ parameter rules the linear combination between $L$ and $G$, so that when $r=0, V(x)$ is a pure Gaussian, and when $r=1, V(x)$ is a pure Lorentzian. The FWHM of $L$ and $G$ is forced to be identical and varies as a function of the EW with the following relation:

$$
\mathrm{FWHM}=\mathrm{FWHM}_{\text {best }}+\mathrm{EW} / 4,
$$

where FWHM $_{\text {best }}$ is the best-matching FWHM found by the minimization routine during the best-matching model searching. Unlike Bruce et al. (2000), we make the parameter $r$ dependent from the EW:

$$
r=0.5 \cdot \exp \left(\frac{-1}{(3 \mathrm{EW})^{2}+0.001}\right)
$$

so that for small EW the line profile is Gaussian and for large EW the line profile approximates a Voigt profile.

Kielkopf (1973) showed that the difference between the real and the pseudo-Voigt profile described by Equation (8) is always smaller than $1.2 \%$ for $\mathrm{EW}=0.5 \AA$, which corresponds to an error smaller than $0.72 \%$ in $\mathrm{EW}$ (Bruce et al. 2000).

\subsection{Improved Continuum Re-normalization}

In order to remove some fringing effects that sometimes affect the initial input RAVE spectra (the same ones as used by the DR4 pipeline; see Section 3.6), the chemical pipeline has its own internal re-normalization algorithm. It can be summarized as follows (a more detailed discussion can be found in Section 2.5 and Figure 3 of B11):

1. A preliminary metallicity estimation is performed and the modeled metallic lines are subtracted from the observed spectrum.

2. The strong lines belonging to the $\mathrm{Ca}$ II and $\mathrm{H}$ I are fitted with a Lorentzian profile and subtracted from the observation.

3. The continuum profile is then defined by a box-car smoothing of the residuals obtained after the previous two steps.

4. The strong $\mathrm{Ca}$ II and $\mathrm{HI}_{\mathrm{I}}$ lines are added to the continuum profile to obtain the new "continuum." The chemical pipeline does not measure the broad lines of the $\mathrm{Ca}$ II and $\mathrm{H}$, and their profiles are considered part of the continuum level. Therefore, by adding them to the classical continuum, they are excluded from the chemical analysis. It is by comparison with this level of "continuum" that the metallic lines are measured.
This re-normalization permits better continuum placement around the absorption lines for a better elemental abundance estimation. In particular, the new adopted Voigt profile (see Section 5.2) contributes to improve the continuum placement thanks to the superior fit of the line's wings, which can now be properly subtracted during the re-normalization procedure.

The present chemical pipeline applies the continuum placement like the chemical pipeline outlined in B11 (see their Section 2.5), with the difference that it is applied twice for spectra with $\mathrm{S} / \mathrm{N} \geqslant 40 \mathrm{pixel}^{-1}$ and only once for $\mathrm{S} / \mathrm{N}<40 \mathrm{pixel}^{-1}$. Indeed, thanks to the pseudo-Voigt profile, the continuum placement process becomes more stable at $\mathrm{S} / \mathrm{N} \geqslant 40 \mathrm{pixel}^{-1}$, and when applied iteratively the continuum estimation converges after two iterations. On the other hand, for S $/ \mathrm{N}<40$ pixel $^{-1}$ the continuum estimation cannot converge to the right level. The noise spikes (mistaken as metallic lines by the code) lead to a too high continuum placement and, consequently, to a too high metallicity estimation. Thus, the re-normalization is applied only once for low-S/N spectra.

\subsection{Precision and Accuracy of the RAVE Chemical Elemental Abundances}

In order to evaluate the precision and accuracy of the new chemical pipeline, we ran tests on synthetic and real spectra with known chemical abundances and compared the results with the expected abundances. The samples of synthetic and real spectra used are the same as those employed in B11. This allows us to have a clear view of the achieved improvement between the two pipelines.

Unlike the work presented in B11, we present here the tests and results for only six elements (aluminum, magnesium, silicon, titanium, nickel, and iron). We rejected the calcium abundance as not being reliable (see Section 6.3.1).

\subsubsection{Internal Errors: Tests on Synthetic Spectra}

The tests have been performed on a sample of 1353 synthetic spectra. The values for the effective temperature and the surface gravity for these spectra have been taken from a mock sample of RAVE observations created using the Besançon model, whereas the adopted chemical abundances have been taken from the Soubiran \& Girard (2005) catalog, whose star metallicities span from -1.5 dex to +0.4 dex (for further details on how the sample has been constructed, see B11). This ensures plausible stellar parameters and chemical abundance distributions of the synthetic spectra.

We evaluated the precision and accuracy of the results at $\mathrm{S} / \mathrm{N}=100,40,20$ pixel $^{-1}$. In Figures 13 and 14 we report the detailed results.

Results at $S / N=100$ pixel $^{-1}$. While the B11 chemical pipeline gave slightly underestimated abundances, the present one reduces or removes such underestimation for most of the elements at $\mathrm{S} / \mathrm{N}=100 \mathrm{pixel}^{-1}$. $[\mathrm{Ni} / \mathrm{H}]$ is underestimated by $\sim 0.1 \mathrm{dex}$, whereas the $[\mathrm{Ti} / \mathrm{H}]$ estimate is good for giants but should be rejected for dwarfs (for which Ti lines are too weak for a good estimation).

Results at $S / N=40$ pixel $^{-1}$. All the elements have reliable abundances, except for $[\mathrm{Si} / \mathrm{H}]$ and $[\mathrm{Ti} / \mathrm{H}]$, which look overestimated by $\sim+0.1$ and $\sim+0.2$ dex, respectively.

Results at $S / N=20$ pixel $^{-1}$. $[\mathrm{Fe} / \mathrm{H}],[\mathrm{Si} / \mathrm{H}]$, and $[\mathrm{Al} / \mathrm{H}]$ are reliable, with uncertainties of $\sim 0.15-0.20 \mathrm{dex}$. $[\mathrm{Mg} / \mathrm{H}]$ and 

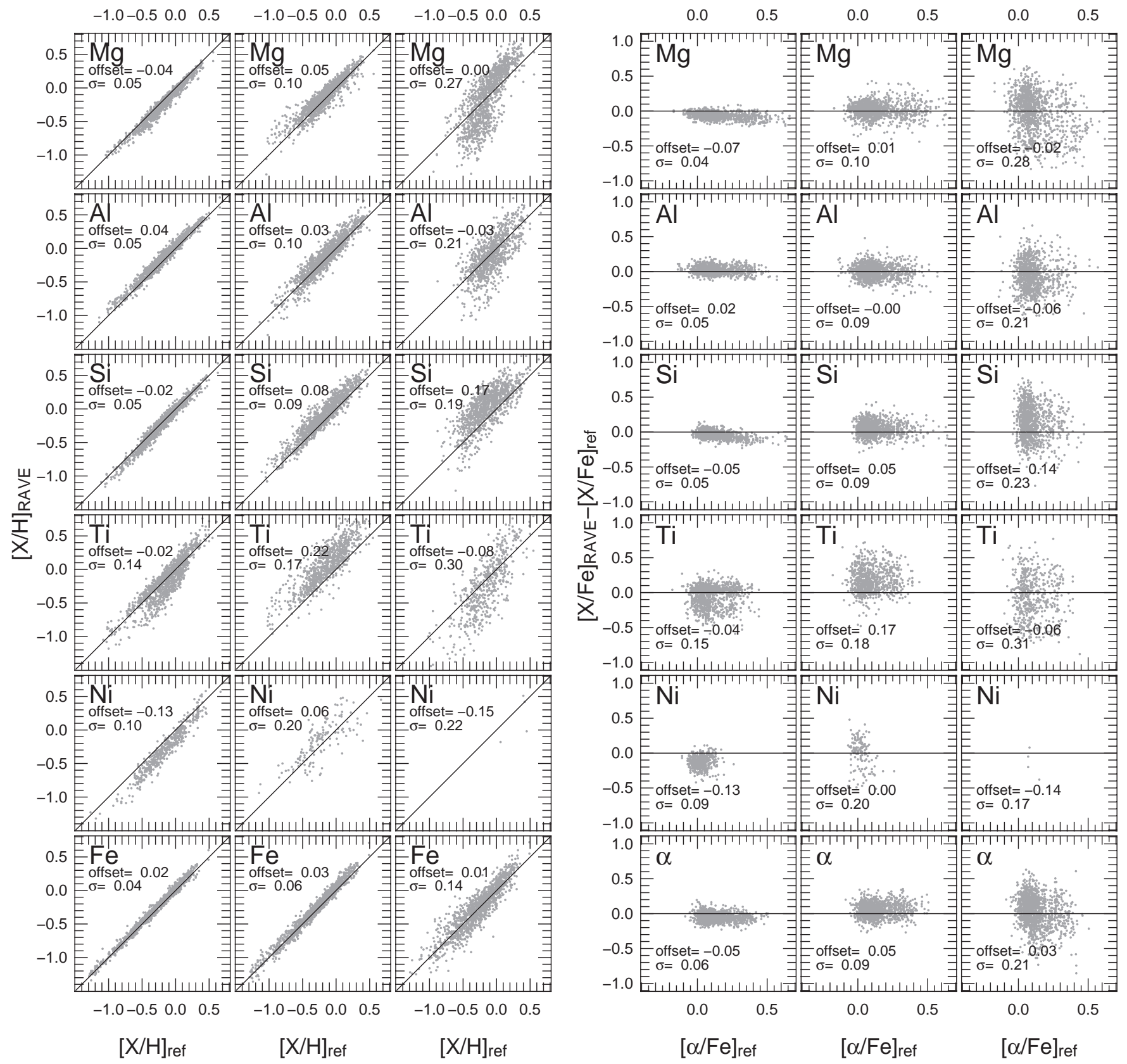

Figure 13. Left: expected elemental abundances $[\mathrm{X} / \mathrm{H}](x$-axis) vs. measured elemental abundances ( $y$-axis) for the sample of synthetic spectra at $\mathrm{S} / \mathrm{N}=100,40$, and $20 \mathrm{pixel}^{-1}$ (for the left, middle, and right column, respectively) and assuming no errors in stellar parameters. Right: as in left panels but for the expected enhancement $[\mathrm{X} / \mathrm{Fe}](x$-axis) and the residuals measured-minus-expected (y-axis). Offsets and standard deviations are reported in the panels. $\alpha$-abundances are computed as in B11, i.e., the mean of $\mathrm{Mg}$ and $\mathrm{Si}$ abundances.

$[\mathrm{Ti} / \mathrm{H}]$ show significant systematics, and $[\mathrm{Ni} / \mathrm{H}]$ cannot be measured because its lines are too weak.

For $\mathrm{Ni}$ and $\mathrm{Ti}$ the selection effect due to the $\mathrm{S} / \mathrm{N}$ is particularly evident. Moving to lower $\mathrm{S} / \mathrm{N}$, the number of spectra with $\mathrm{Ti}$ and $\mathrm{Ni}$ estimations decreases, because the lines of $\mathrm{Ti}$ (in dwarfs stars) and $\mathrm{Ni}$ are weak in the RAVE wavelength range and do not overcome the noise at low $\mathrm{S} / \mathrm{N}$. This selection bias is further discussed in Section 6.3.1.

In general, the new chemical pipeline suffers smaller systematics with respect to the old one. Underestimations are reduced, and abundances of important elements $(\mathrm{Fe}, \mathrm{Si}, \mathrm{Al}$, and $\mathrm{Mg}$ ) do not correlate with the effective temperature (see Figure 15) as they did with the previous pipeline. $[\mathrm{Ti} / \mathrm{H}]$ appears reliable only for cool giants.
We further tested the robustness of our results by repeating the abundance measurements after adjusting randomly the initial $T_{\text {eff }}, \log g$, and $[\mathrm{M} / \mathrm{H}]$ by values normally distributed around their true values with $\sigma_{\mathrm{T}_{\mathrm{eff}}}=250 \mathrm{~K}, \sigma_{\log g}=0.5 \mathrm{dex}$, and $\sigma_{[\mathrm{M} / \mathrm{H}]}=0.2 \mathrm{dex}$, respectively. The results are shown in Figure 14. The shifts in stellar parameters (representing the input errors) simply inflate the errors in abundances seen in the test without stellar parameters errors, without introducing any new systematics.

\subsubsection{External Errors: Tests on Real Spectra}

The overall uncertainties have been estimated by computing the elemental abundances of 98 RAVE spectra of dwarf stars from Soubiran \& Girard (2005, hereafter SG05) and 233 

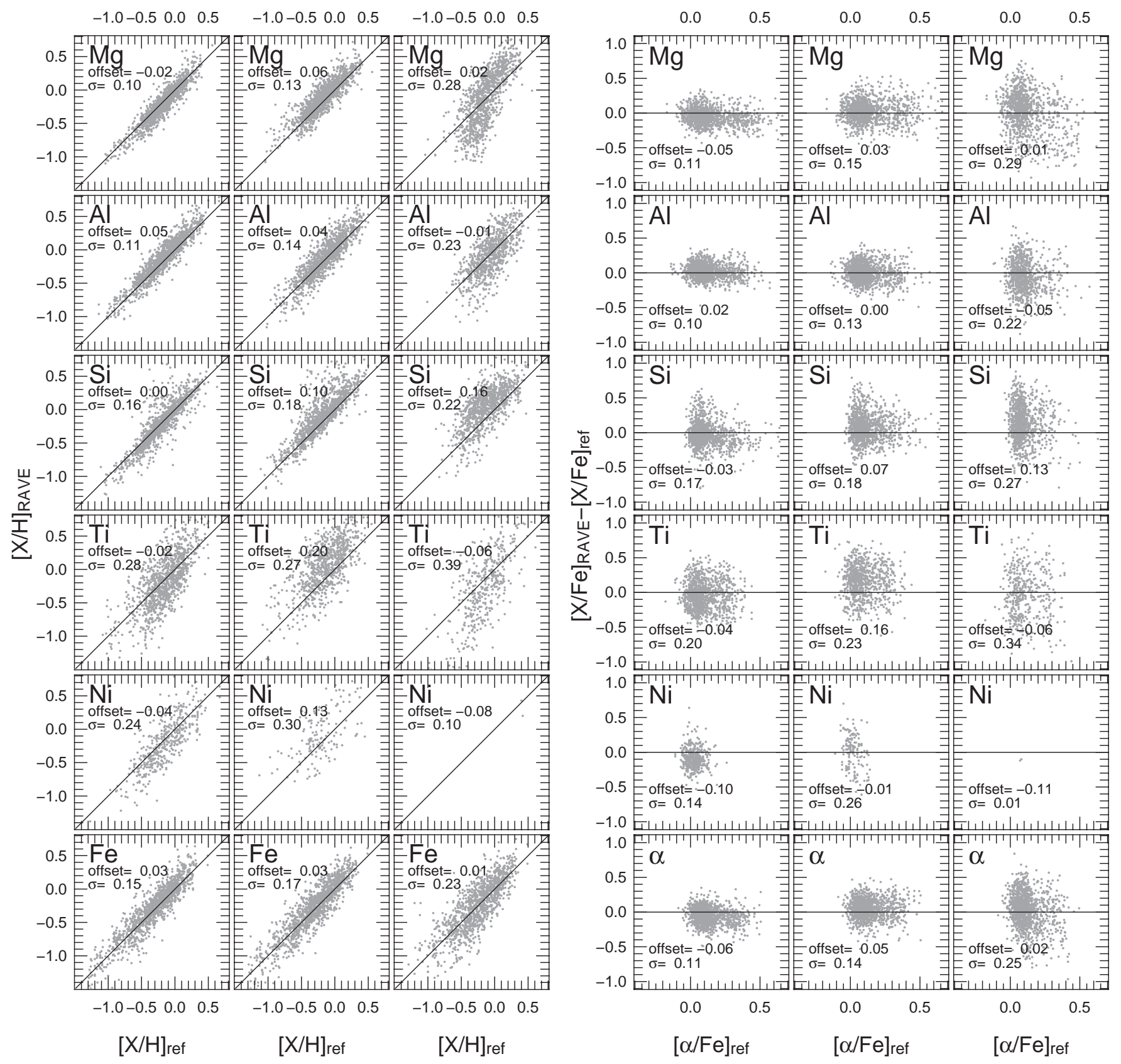

Figure 14. As in Figure 13 but with noisy stellar parameters to simulate the errors appropriate for the RAVE pipeline.

RAVE spectra of 203 giant stars from Ruchti et al. (2011, hereafter R11). Most of the SG05 stars have RAVE spectra with $\mathrm{S} / \mathrm{N}>100$ pixel $^{-1}$, whereas the R11 stars have RAVE spectra with $\mathrm{S} / \mathrm{N}$ ranging between 30 pixel $^{-1}$ and 90 pixel $^{-1}$. Hence, the results are representative of the medium-high $\mathrm{S} / \mathrm{N}$ regime. Figures 16 and 17 show the results obtained for the six elements in common with SG05 and the four in common with R11.

Adopting the RAVE DR4 stellar parameters, the RAVE chemical pipeline delivers slightly underestimated abundances for $\mathrm{Mg}, \mathrm{Al}$, and $\mathrm{Ti}(\sim-0.1 \mathrm{dex})$. There is a general improvement in precision for most of the elements with respect to the B11 pipeline (dispersions smaller than $\sim 0.05-0.07 \mathrm{dex}$ for $\mathrm{Mg}, \mathrm{Ti}$, and $\mathrm{Fe}$ ) with no visible systematic offsets. The estimated errors in abundance depend on the element and range from $0.17 \mathrm{dex}$ for $\mathrm{Mg}, \mathrm{Al}$, and $\mathrm{Ti}$ to $0.3 \mathrm{dex}$ for $\mathrm{Ti}$ and $\mathrm{Ni}$. The error for $\mathrm{Fe}$ is estimated as 0.23 dex. We note that the errors reported here are conservative estimations of the RAVE abundance errors, because we are comparing our results with other more precise but still uncertain measurements, and we have not corrected the variance for the second contribution. For illustration, assuming an uncertainty in the reference abundances of $0.1 \mathrm{dex}$, our estimated RAVE errors decrease by $0.03-0.05$ dex.

\section{FOURTH PUBLIC DATA RELEASE: CATALOG PRESENTATION}

The fourth public data release of the RAVE data (RAVE DR4) includes the observations obtained from the 2004 April 3 to the 2012 December 20. In total, 425,561 stars have been observed, collecting 482,430 spectra. The catalog is accessible online, and it contains also radial velocities, proper motions, photometric information, stellar morphological flags (coming from Matijevič et al. 2012), line-of-sight distances, ages, and 

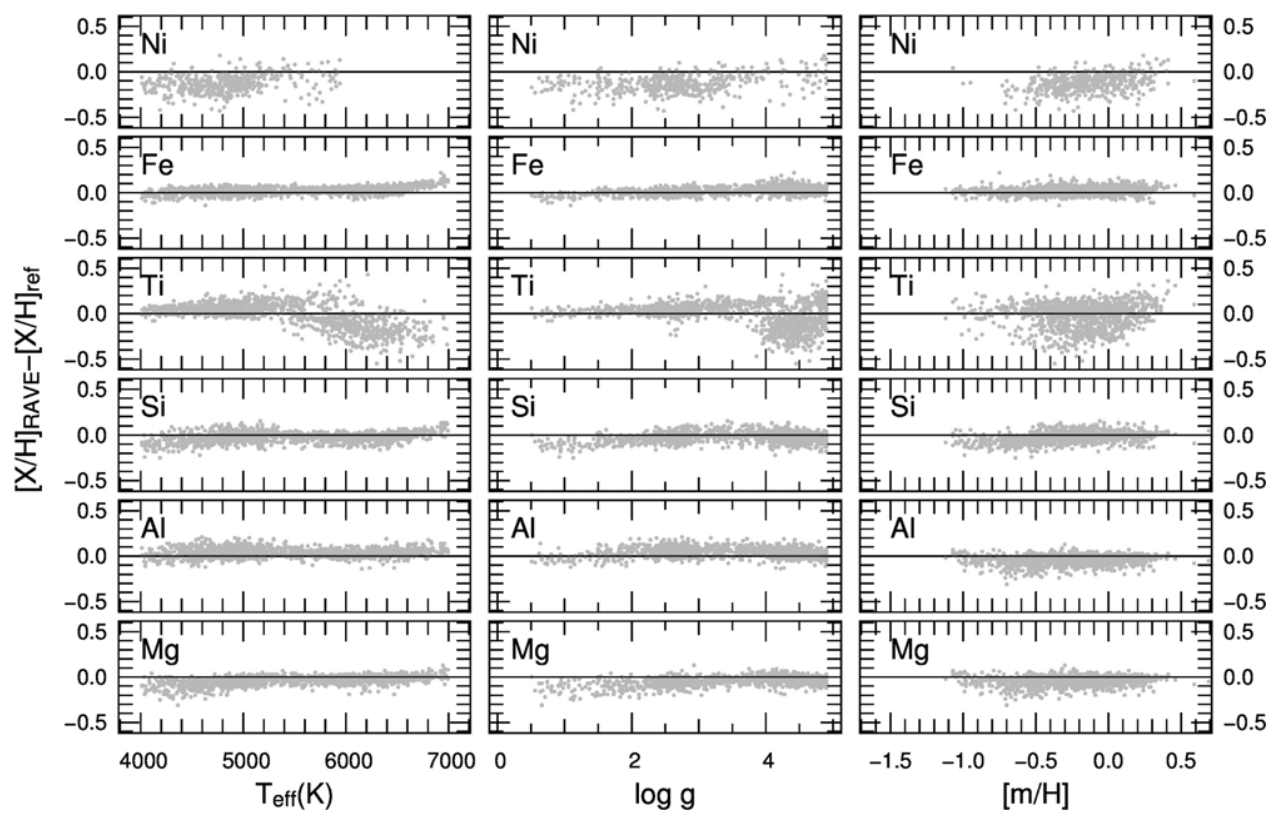

Figure 15. Correlation between the elemental abundance residuals and the stellar parameters at $\mathrm{S} / \mathrm{N}=100 \mathrm{pixel}^{-1}$.
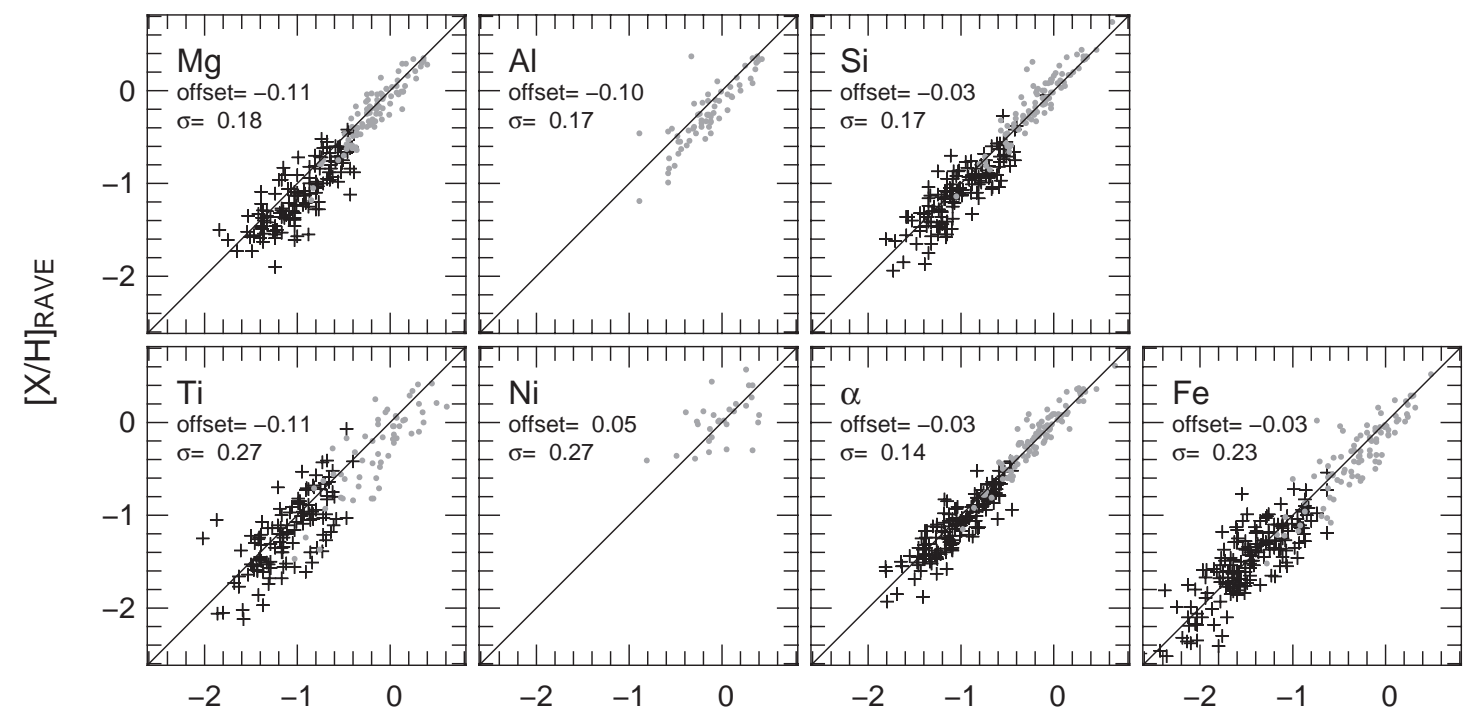

$[\mathrm{X} / \mathrm{H}]_{\mathrm{ref}}$

Figure 16. Comparison between the reference high-resolution elemental abundances ( $x$-axis) and RAVE elemental abundances ( $y$-axis) for the SG05 (98 dwarf stars, gray dots) and the R11 samples ( 233 spectra of 203 giant stars, black "+") measured by adopting the stellar parameters provided by the RAVE pipeline.

interstellar extinction for each star. In addition, the parameters obtained with the previous DR3 pipeline are also published, to assist readers of papers published based on those parameters, though we strongly recommend the use of the parameters obtained with the latest DR4 pipeline in all future analyses. The DR4 catalog can be queried or retrieved from the Vizier database at the Centre de Données Astronomiques de Strasbourg (CDS), as well as from the RAVE collaboration Web site (www.rave-survey.org).

The completeness of the published catalog for the radial velocities, atmospheric parameters, distances, and chemical abundances with respect to the $I_{2 \text { MASS }}$ catalog can be seen in Figure 18. In addition, Aitoff maps for the completeness of the catalog at four different magnitude bins as a function of the stellar positions on the sky are shown in Figure 3.

Below we discuss which criteria to apply in order to obtain a high-quality and reliable sample of stars from the catalog of the atmospheric parameters and the elemental abundances. Brief discussions about proper motions, radial velocities, distances, and the new APASS photometry are also included in what follows, but we refer the reader to Siebert et al. (2011b), Zwitter et al. (2010), and Binney et al. (2013) for full details.

\subsection{Criteria for Reliable Sub-sample Selection Considering the Atmospheric Parameters}

The following criteria need to be understood as the confidence limits for selection based on observational $(\mathrm{S} / \mathrm{N})$ and pipeline limitations (mainly the boundaries of the grid).

We selected all the stars that had S/N > 20 pixel $^{-1}$, had errors in the RV estimation of less than $8 \mathrm{~km} \mathrm{~s}^{-1}$ (measured by RAVE DR3; see Section 8), had derived $\log g>$ 0.5 dex, determined $T_{\text {eff }}>3800 \mathrm{~K}$, and calibrated metallicity $[\mathrm{M} / \mathrm{H}]>-5 \mathrm{dex}$ (measured by the DR4 pipeline), and for which 


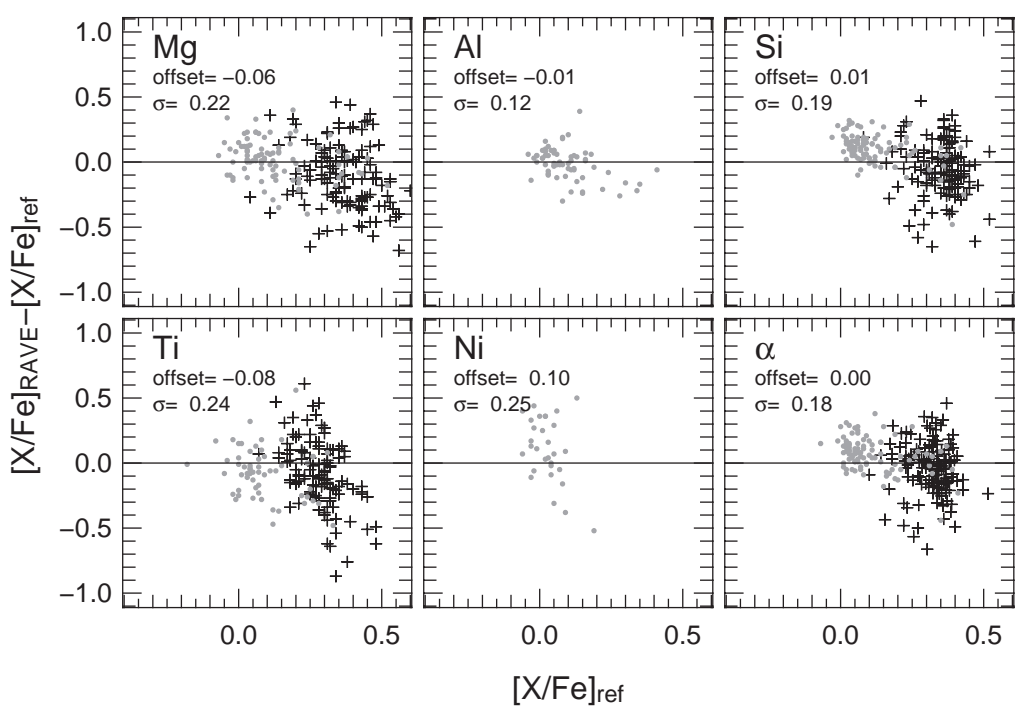

Figure 17. Comparison between expected relative elemental abundance ( $x$-axis) and residual abundances RAVE-minus-reference ( $y$-axis). Stellar parameters adopted and symbols are as in Figure 16.

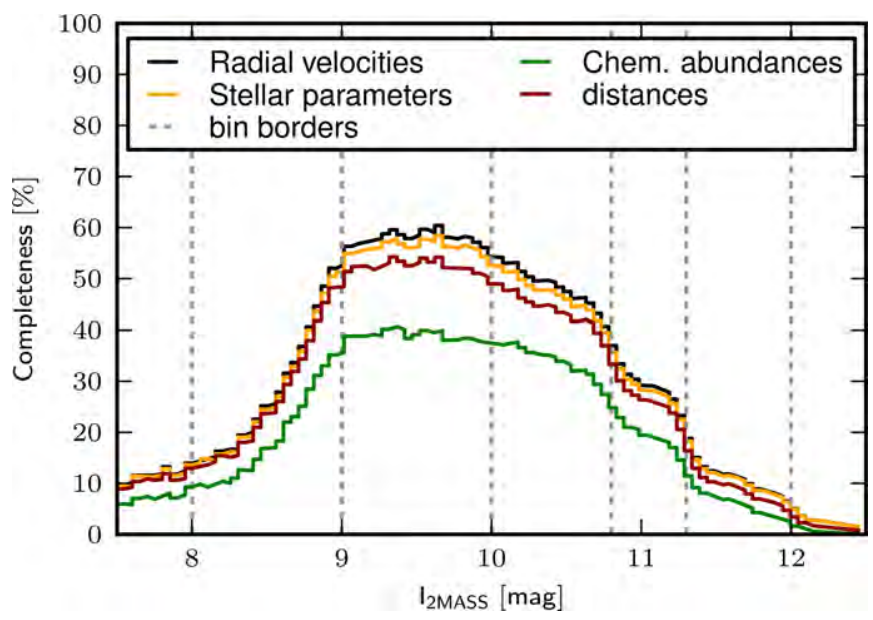

Figure 18. Fractional completeness of the RAVE DR4 sample with respect to the $I_{2 M A S S}$ stars for the published radial velocities, stellar atmospheric parameters, chemical abundances, and line-of-sight distances.

(A color version of this figure is available in the online journal.)

the DR4 algorithm had converged toward a stable solution. ${ }^{33}$ In total, roughly $19 \%\left(\sim 8.7 \times 10^{4}\right)$ of the spectra have been rejected after these quality criteria. We are working toward the next DR5 RAVE data release, with work that we hope will improve the parameters for at least some of these currently non-reliable stars.

The cut on the error on the RV $\left(\Delta V_{\mathrm{HRV}}<8 \mathrm{~km} \mathrm{~s}^{-1}, 12,974\right.$ spectra) has been defined based on the results of Kordopatis et al. (2011a), where it has been shown that for Doppler shifts larger than approximately half a pixel, the results of the pipeline were seriously degraded. Nevertheless, a criterion based on the Tonry-Davis correlation coefficient $(R)$ might be preferred in some cases, since some stars can have large errors but good $R$ (due, for example, to strong hydrogen lines) and vice versa (small errors but $R<5$ ).

The removal of the stars with gravities lower or equal to 0.5 dex $(25,882$ spectra $), T_{\text {eff }}$ lower than $3800 \mathrm{~K}(20,143$ spectra), and/or calibrated $[\mathrm{M} / \mathrm{H}]$ lower than $-5 \operatorname{dex}(1282$

33 MATISSE iterates up to 10 times until the result of the projection of the spectrum on the projection functions $B_{\theta}(\lambda)$ is within the parameter range defined by the $B_{\theta}(\lambda)$ (see Recio-Blanco et al. 2006; Kordopatis et al. 2011a, and Section 3.3) spectra) has been decided because the results are considered both unrealistic (the synthetic spectra computed with the MARCS atmospheric models at such $\log g$ have not been carefully compared to real spectra) and less reliable (e.g., missing models in the reference grid). Finally, the cut on the convergence of the DR4 algorithm (14,454 spectra) is made in order to minimize cases badly affected by the spectral degeneracies. Indeed, these degeneracies can cause, in some cases, an impossibility for the algorithm to converge due to a negative gradient in the distance function between the spectrum and the templates. MATISSE can in some cases oscillate between two solutions ( $\sim 11 \%$ of the published sample). We decided to keep these solutions because, in general, they are close in the parameter space. Nevertheless, in case the user decides not to use them, we have flagged these stars in the algo_conv parameter, which is also published with this data release (see the Appendix).

An additional cut, based on the velocity width parameter of the spectral lines, $V_{\text {rot }}$, has been applied, since our algorithm cannot treat fast rotators. We discarded empirically stars at the high-velocity tail of the distribution $\left(V_{\text {rot }}>100 \mathrm{~km} \mathrm{~s}^{-1}\right.$, 11,735 in total). We recall that the estimation of the $V_{\text {rot }}$ is made through the DR3 pipeline, as a free parameter, at the same moment as the first estimation of $T_{\text {eff }}, \log g$, and $[\mathrm{M} / \mathrm{H}]$ is made. Nevertheless, the rather low resolving power of RAVE spectra $\left(\sim 1.2 \AA\right.$ or $\left.30 \mathrm{~km} \mathrm{~s}^{-1}\right)$ does not allow the determination of rotational velocities for slow rotators, which represent the vast majority of RAVE stars. Hence, this parameter is not published, but true fast rotators will be discussed in a separate paper.

Finally, we note that targets at low Galactic latitudes should also be treated with caution, since the possibly high interstellar extinctions in these directions are not taken into account in the photometric constraints imposed by the DR4 pipeline.

\subsection{Criteria for Reliable Sub-sample Selection Considering the Chemical Abundances}

From the whole RAVE internal data set, we measured chemical abundances only for spectra with the following features:

1. Effective temperature $4000 \leqslant T_{\text {eff }}(\mathrm{K}) \leqslant 7000 \mathrm{~K}$

2. $\mathrm{S} / \mathrm{N}>20$ pixel $^{-1}$

3. Rotational velocity $V_{\text {rot }}<50 \mathrm{~km} \mathrm{~s}^{-1}$. 


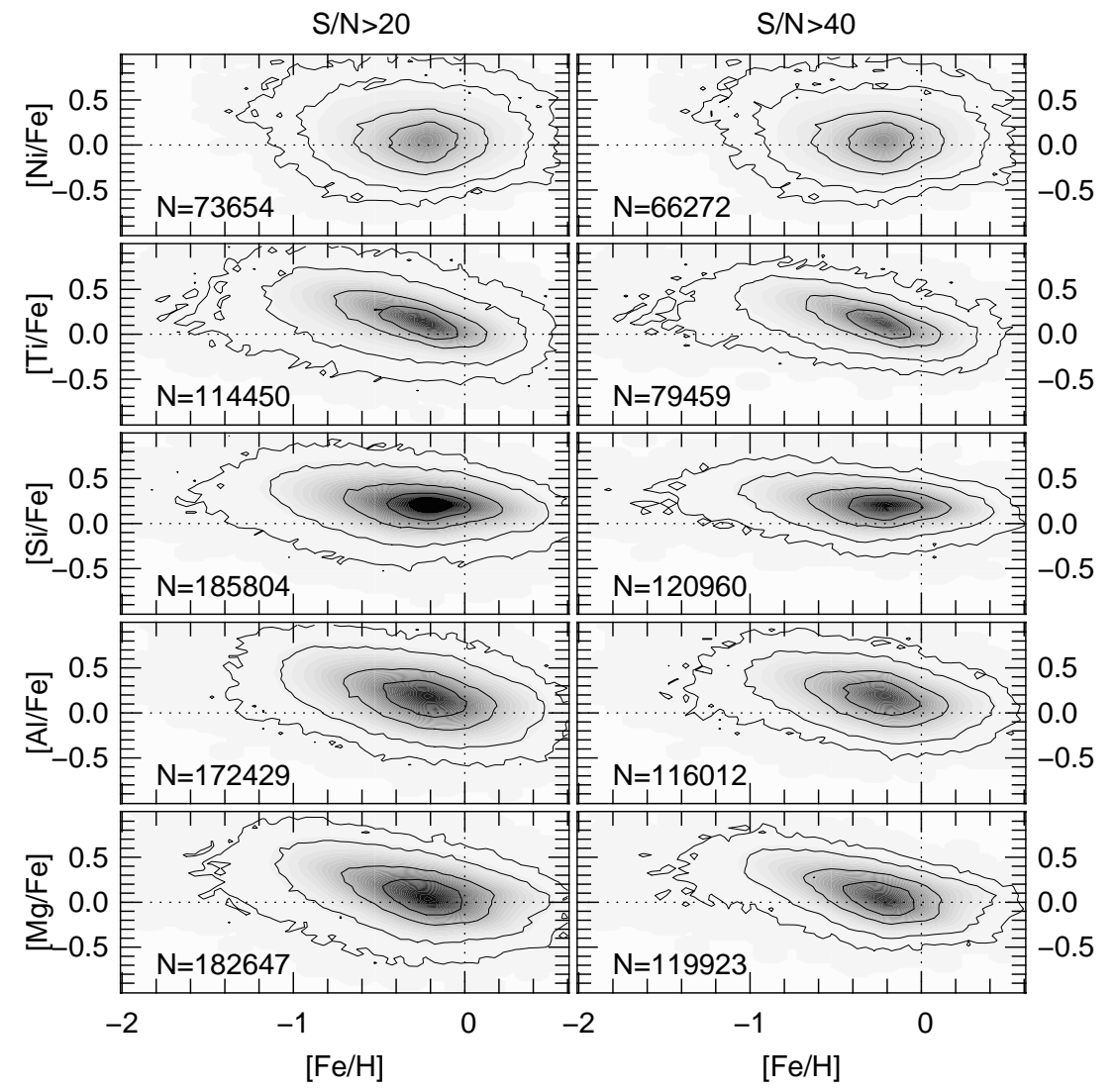

Figure 19. Distribution on the chemical plane of spectra after the application of the quality indicators reported in Section 6.2. The isocontours hold 34.0\%, 68.0\%, $95.0 \%$, and $99.5 \%$ of the sample.

Such limitations are due to the following facts. First, the EW library (and the B11 line list on which it is based) is reliable only in this effective temperature range. In addition, the line measurement and stellar parameters are reliable only for $\mathrm{S} / \mathrm{N}$ larger than 20 pixel $^{-1}$. Finally, the absorption lines can be reliably measured only if their FWHM does not significantly exceed the RAVE instrumental FWHM $(\sim 1.2 \AA)$, which corresponds to a rotational velocity of $30 \mathrm{~km} \mathrm{~s}^{-1}$. Such criteria leave 313,874 spectra selected from the RAVE database.

Besides the chemical abundances of this selected sample, we provide some extra statistical quantities and flags to be employed for further quality selection:

1. $\chi^{2}$ between best-matching model and observed spectrum. The lower the values, the better the expected abundance precision. We suggest a user reject spectra with $\chi^{2}>2000$.

2. The value frac, which represents the fraction of the observed spectrum that satisfactorily matches the model. We suggest a user reject spectra with frac $<0.7$ (see B11 for further details).

3. Classification flags by Matijevič et al. (2012). We suggest a user select spectra classified as "normal" by Matijevic et al. in order to avoid peculiar objects on which the chemical pipeline fails.

4. Algo_Conv value. This value indicates if the DR4 pipeline has converged or if the stellar parameters were either outside the grid boundaries or MATISSE was oscillating between two values. The higher quality data have Algo_Conv $=0$.

The application of these quality flags is left to the user. The number of spectra that meet all such quality flags is 187,305 . In Figure 19 we show the distribution of the chemical abundances, given the above-mentioned criteria, for $\mathrm{S} / \mathrm{N}>20 \mathrm{pixel}^{-1}$ and $\mathrm{S} / \mathrm{N}>40 \mathrm{pixel}^{-1}$.

\subsection{Results and Comparisons with DR3}

A description of Galactic properties based on the published parameters of this catalog is beyond the scope of this paper. Nevertheless, as a sanity check, we explore in this section the general properties of the catalog, by analyzing the correlation of the parameters and the change of the metallicity properties according to the $\mathrm{S} / \mathrm{N}$, the effective temperature, or the surface gravity. By comparing the behaviors of the DR3 and the DR4 pipelines, we show that although the differences between the atmospheric parameters of the two methods are relatively subtle, DR4 better reproduces the expected behavior for different subpopulations of stars and thus is the method of choice for most Galaxy evolution studies.

Figure 20 compares the resulting $T_{\text {eff }}-\log g$ diagrams of the DR4 and the DR3 pipelines as selected according to the criteria of Section 6.1. One can notice that besides the well-understood and described discretization due to the DEGAS algorithm, there are some additional subtle differences in the parameters of the two pipelines. In particular, hot dwarfs, as well as turn-off stars, have now smaller surface gravities and the main sequence is better defined. Finally, giants have slightly higher effective temperatures.

The DR4 and DR3 ${ }^{34}$ calibrated metallicity trends, as a function of the surface gravity and the effective temperatures, can be seen in Figure 21. As far as the $T_{\text {eff }}$ dependencies

\footnotetext{
${ }^{34}$ Calibrated according to Equation (2) of Siebert et al. (2011b), with $c_{0}=0.578, c_{1}=1.095, c_{3}=1.246, c_{4}=-0.520$.
} 

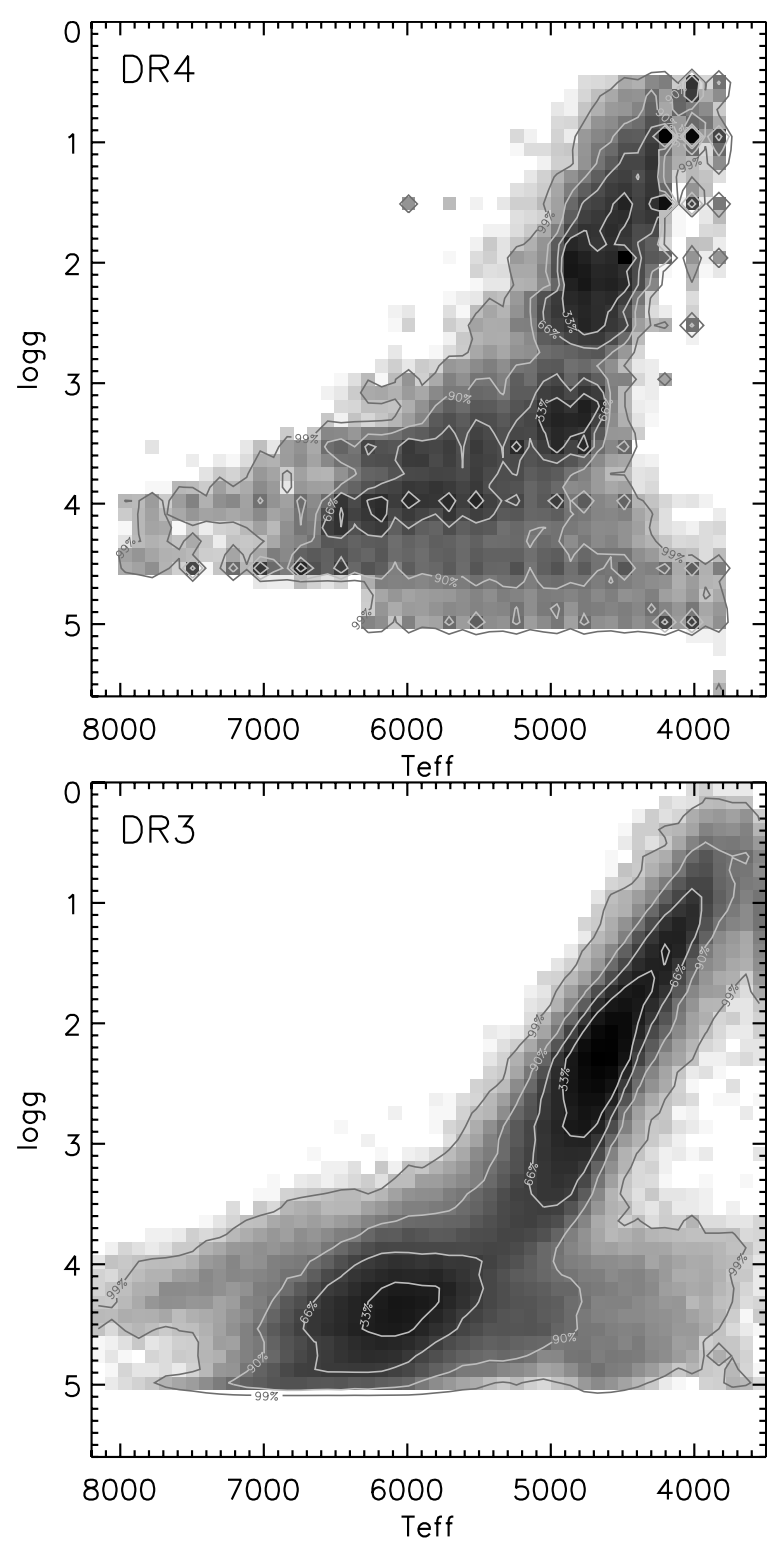

Figure 20. $T_{\text {eff }}-\log g$ diagram for the selected RAVE stars (top: DR4 pipeline; bottom: DR3 pipeline), meeting our criteria defined in Section 6.1. In total the parameters of $\sim 4 \times 10^{5}$ spectra are represented in these diagrams. The contour lines contain $33 \%, 66 \%, 90 \%$, and $99 \%$ of the total considered sample.

are concerned, one can notice that the metallicity distribution functions (MDFs) of the DR4 pipeline get broader when the effective temperature lowers. In particular, the DR4 pipeline finds that the hottest stars have a narrow metallicity distribution with a mean value at slightly super-solar values, as expected for the young stars in the solar neighborhood. This is not the case for the results of the DR3 pipeline, where metallicities as low as $[\mathrm{M} / \mathrm{H}] \sim-0.5$ dex are obtained. Furthermore, from the isocontours of the $\log g$ versus $[\mathrm{M} / \mathrm{H}]$, we can see that despite the mild pixelization of the values, there are no trends of the metallicities as a function of the surface gravity for the dwarfs, as derived by the DR4 pipeline. This is not the case for the DR3 pipeline results, where a shift is noticed.

In order to investigate whether this shift is real and justified given our classical view of the Milky Way, we explored the stellar heliocentric radial velocities and the evolution of the MDFs for different surface gravity bins. Figure 22 shows the resulting histograms for the calibrated metallicities of the DR4 (in black solid lines) and the DR3 pipelines (red dashed lines). The RV dispersions of the selected stars have also been reported inside each box. For the lower panels, corresponding to the dwarf stars $(3.5<\log g<5$ dex $)$, the RV dispersion stays constant. Considering that each Galactic population (thin disk, thick disk, and halo) is characterized by a different velocity dispersion, the constant $\sigma_{V_{\mathrm{HRV}}}$ that is found indicates that the same proportions of Galactic populations are probed for these gravity bins. As a result, the MDFs should not vary inside these bins. This is the case only for the DR4 MDFs, the DR3 ones shifting by $0.2 \mathrm{dex}$ in this range of $\log g$. As far as the sub-giant and giant stars are concerned, a good agreement is found between the DR3 and DR4 MDFs, with a shift toward lower metallicities with decreasing $\log g$ and at the same time an increase in the RV dispersion. This is in agreement with a change in the mixture of the probed Galactic populations as a function of the probed volume, passing from an old thin disk dominated population to the presence of more halo stars for the larger volume probed by the more luminous giant stars.

To show the correlations between the parameters, we select among the DR4 catalog those stars that are observed multiple times, and for which several independent spectra and derived parameter sets are available. In the panels we plot the differences between the several determinations of the measured $T_{\text {eff }}, \log g$, and calibrated metallicities. Figure 23 shows the results for the stars with $\mathrm{S} / \mathrm{N}>20 \mathrm{pixel}^{-1}$, for both DR4 and DR3 pipelines. From that figure one can see that the new DR4 pipeline is more robust than the DR3 one, since the bulk of the stellar parameters show a very small discrepancy between the repeated observations, as well as a negligible parameter correlation. This validates once more the robustness of our calibration relation of Equation (7). However, we note that correlations between the parameter estimations still exist for some stars. This is expected, due to the intrinsic spectral degeneracy: an underestimation of the $T_{\text {eff }}$ leads to a similar underestimation of the $\log g$ and the $[\mathrm{M} / \mathrm{H}]$.

The robustness of the DR4 pipeline in terms of better treatment of the spectral degeneracies can also be seen in Figure 24. The evolution of the mean metallicity as a function of the S/N (yellow points on Figure 24) shows no trends down to $\mathrm{S} / \mathrm{N}>15 \mathrm{pixel}^{-1}$. Nevertheless, the cost for this better treatment is the pixelation of the results for the most metal-poor stars or the ones having low $\mathrm{S} / \mathrm{N}$. In particular, the pixelization effect can be clearly seen in Figure 24 below the threshold of $\mathrm{S} / \mathrm{N}=30 \mathrm{pixel}^{-1}$. Indeed, we recall that for all the observed spectra DEGAS is first used to find the nominal template spectrum of the learning grid in order to re-normalize the observed one. Then, on one hand, for the high-S/N regime, the MATISSE algorithm is used on these optimally re-normalized spectra in order to obtain the final parameters. On the other hand, for the low-S/N data $\left(<30 \mathrm{pixel}^{-1}\right)$ and at the boundaries of the learning grid, tests on synthetic spectra have shown that the projection method was giving less accurate results than the decision tree. Given these facts, Kordopatis et al. (2011a) showed that DEGAS is preferred over the projection approach of MATISSE for $\mathrm{S} / \mathrm{N}<30 \mathrm{pixel}^{-1}$, even if a pixelization of the parameters is introduced.

\subsubsection{Chemical Abundance Reliability: Element by Element}

Besides the quality indicators described in Section 6.2, the number of measured absorption lines for an element can be a good indicator of the reliability and precision of the abundance 

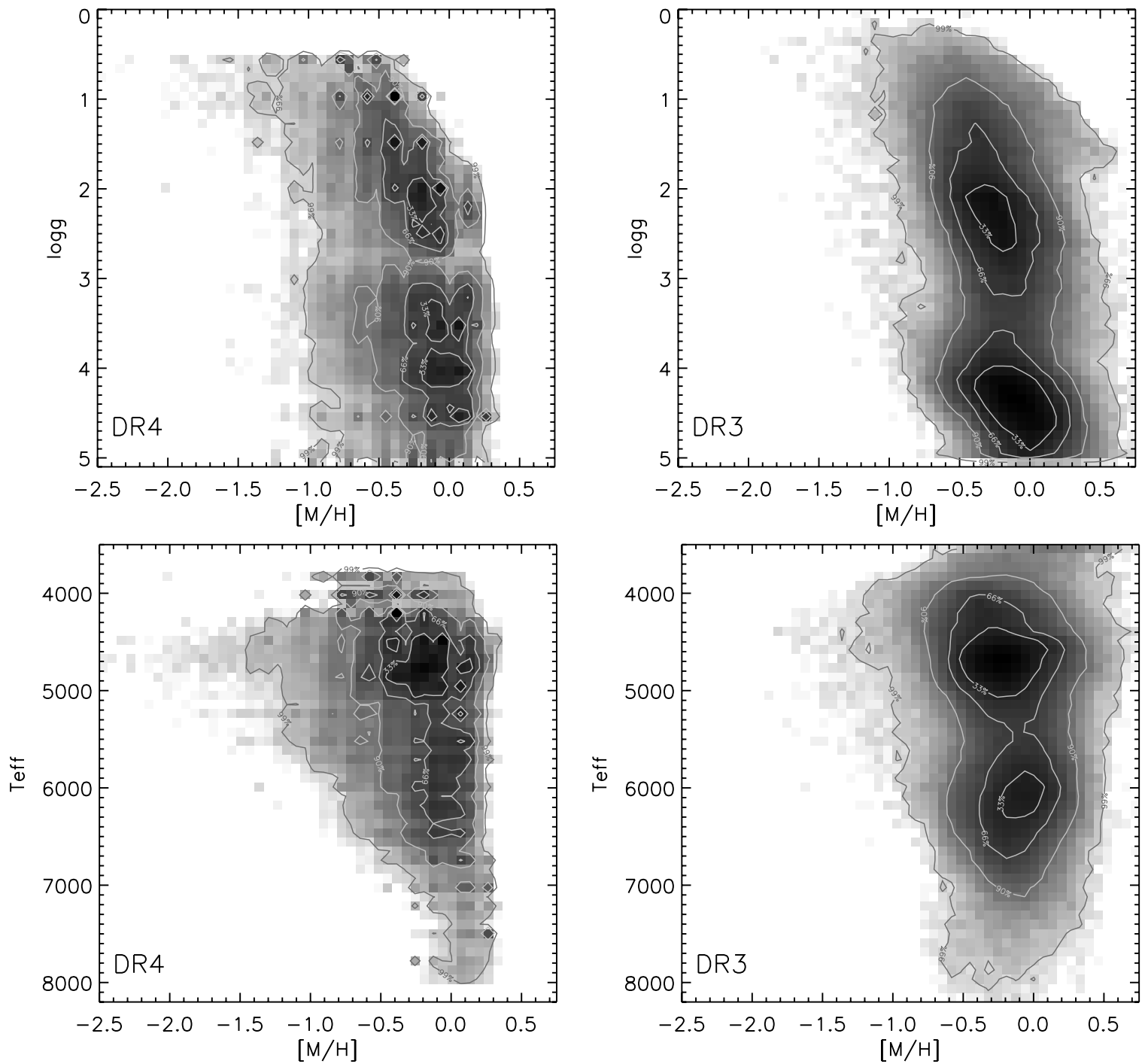

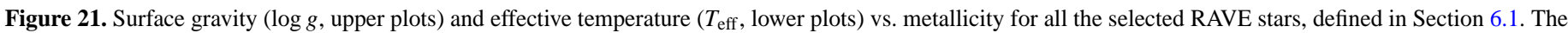

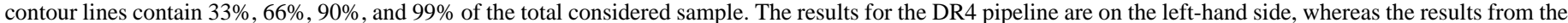

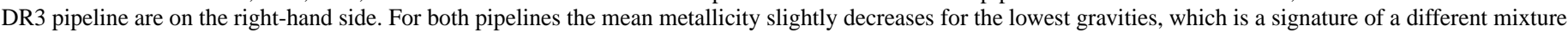
in the probed Galactic populations. In addition, one can notice the $\log g$ and the $T_{\text {eff }}$ trends that are present for the dwarfs analyzed with the DR3 pipeline.

estimation (as illustrated in Figure 25). We outline here a summary of the expected precision of the abundance element by element.

Magnesium yields reliable results on synthetic and real spectra with no significant correlation with stellar parameters. We expect errors $\sigma_{\mathrm{Mg}} \leqslant 0.15 \mathrm{dex}$ for $\mathrm{S} / \mathrm{N}>40 \mathrm{pixel}^{-1}$ and $\sigma_{\mathrm{Mg}} \sim 0.25$ dex for $20<\mathrm{S} / \mathrm{N}<40 \mathrm{pixel}^{-1}$. Tests on synthetic spectra show that magnesium suffers a systematic error when measured at low $\mathrm{S} / \mathrm{N}$. Such an error has not been confirmed with real spectra.

Aluminum gives a reliable abundance although obtained with only two isolated lines. Abundance errors expected are $\sigma_{\mathrm{Al}} \leqslant 0.15 \mathrm{dex}$ for $\mathrm{S} / \mathrm{N}>40 \mathrm{pixel}^{-1}$ and $\sigma_{\mathrm{Al}} \sim 0.25 \mathrm{dex}$ for $20<\mathrm{S} / \mathrm{N}<40$ pixel $^{-1}$.

Silicon is among the most reliably determined elements. Abundance errors expected are $\sigma_{\mathrm{Si}} \leqslant 0.15$ dex for $\mathrm{S} / \mathrm{N}>$ 40 pixel $^{-1}$ and $\sigma_{\mathrm{Si}} \sim 0.25 \mathrm{dex}$ for $20<\mathrm{S} / \mathrm{N}<40 \mathrm{pixel}^{-1}$ with a small overestimation of $\sim 0.1 \mathrm{dex}$.

Titanium gives reliable estimates at high $\mathrm{S} / \mathrm{N}$ for cool giants $\left(T_{\text {eff }}<5500 \mathrm{~K}\right.$ and $\left.\log g<3\right)$. We suggest rejecting $\mathrm{Ti}$ abundances for dwarf stars. Tests on synthetic and real spectra suggest an expected error of $\sigma_{\mathrm{Ti}} \leqslant 0.2 \mathrm{dex}$ for $\mathrm{S} / \mathrm{N}>40 \mathrm{pixel}^{-1}$ and $\sigma_{\mathrm{Ti}} \sim 0.3$ dex for $20<\mathrm{S} / \mathrm{N}<40 \mathrm{pixel}^{-1}$.

Iron gives robust and precise abundances thanks to its large number of measurable lines at all stellar parameter values. Expected errors are $\sigma_{\mathrm{Fe}} \leqslant 0.1$ dex for $\mathrm{S} / \mathrm{N}>40$ pixel $^{-1}$ and $\sigma_{\mathrm{Fe}} \sim 0.2$ dex for $20<\mathrm{S} / \mathrm{N}<40 \mathrm{pixel}^{-1}$.

Nickel abundances have to be used with care because of the few lines that are measurable. From synthetic spectra we infer that $\mathrm{Ni}$ should be used for cool stars only $\left(T_{\text {eff }}<5000 \mathrm{~K}\right)$ and high $\mathrm{S} / \mathrm{N}$. In this regime, the abundances are reliable (despite being underestimated by $\sim 0.1 \mathrm{dex}$ ) with an expected error of $\sigma_{\mathrm{Ni}} \sim 0.25$ dex. The mean abundance correlates with the number of measured lines (i.e., with $\mathrm{S} / \mathrm{N}$ ) as highlighted in Figure 25.

$\alpha$-enhancement is the average of $[\mathrm{Mg} / \mathrm{Fe}]$ and $[\mathrm{Si} / \mathrm{Fe}]$, and it proved to be a robust estimation, particularly useful at low $\mathrm{S} / \mathrm{N}$, where the measurements are more uncertain. The expected error is $\sim 0.15$ dex for $\mathrm{S} / \mathrm{N}>40 \mathrm{pixel}^{-1}$ and $\sim 0.2$ dex for $20<$ $\mathrm{S} / \mathrm{N}<40$ pixel $^{-1}$. 


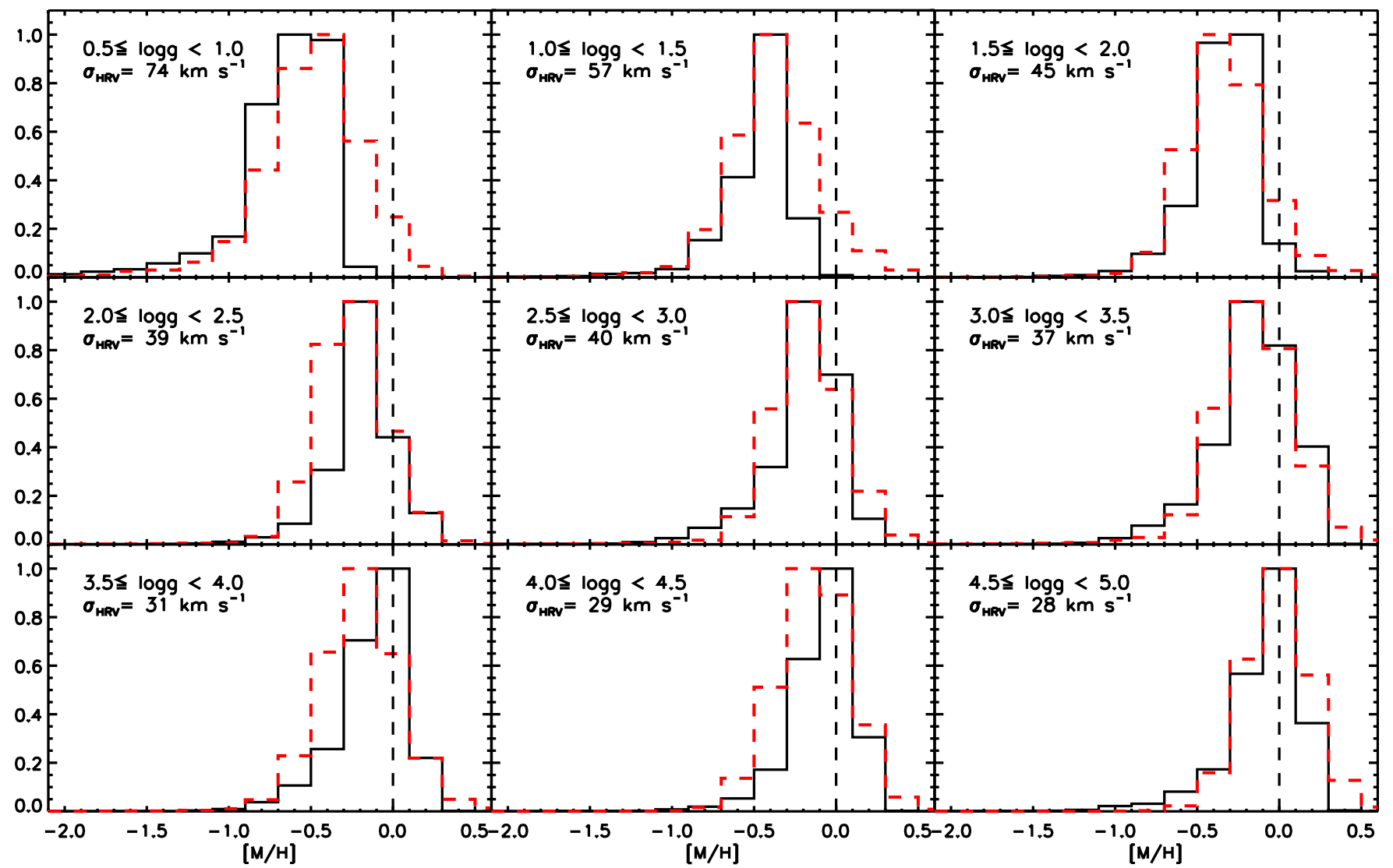

Figure 22. Calibrated metallicity distribution functions for different surface gravity $(\log g)$ bins. The peaks of the histograms have been normalized to unity. The mean radial velocity dispersion for the selected stars per surface gravity bin $\left(\sigma_{\mathrm{HRV}}\right)$ is noted in the upper part of each box. RAVE DR4 results are plotted in black solid lines, RAVE DR3 ones in red dotted lines.

(A color version of this figure is available in the online journal.)
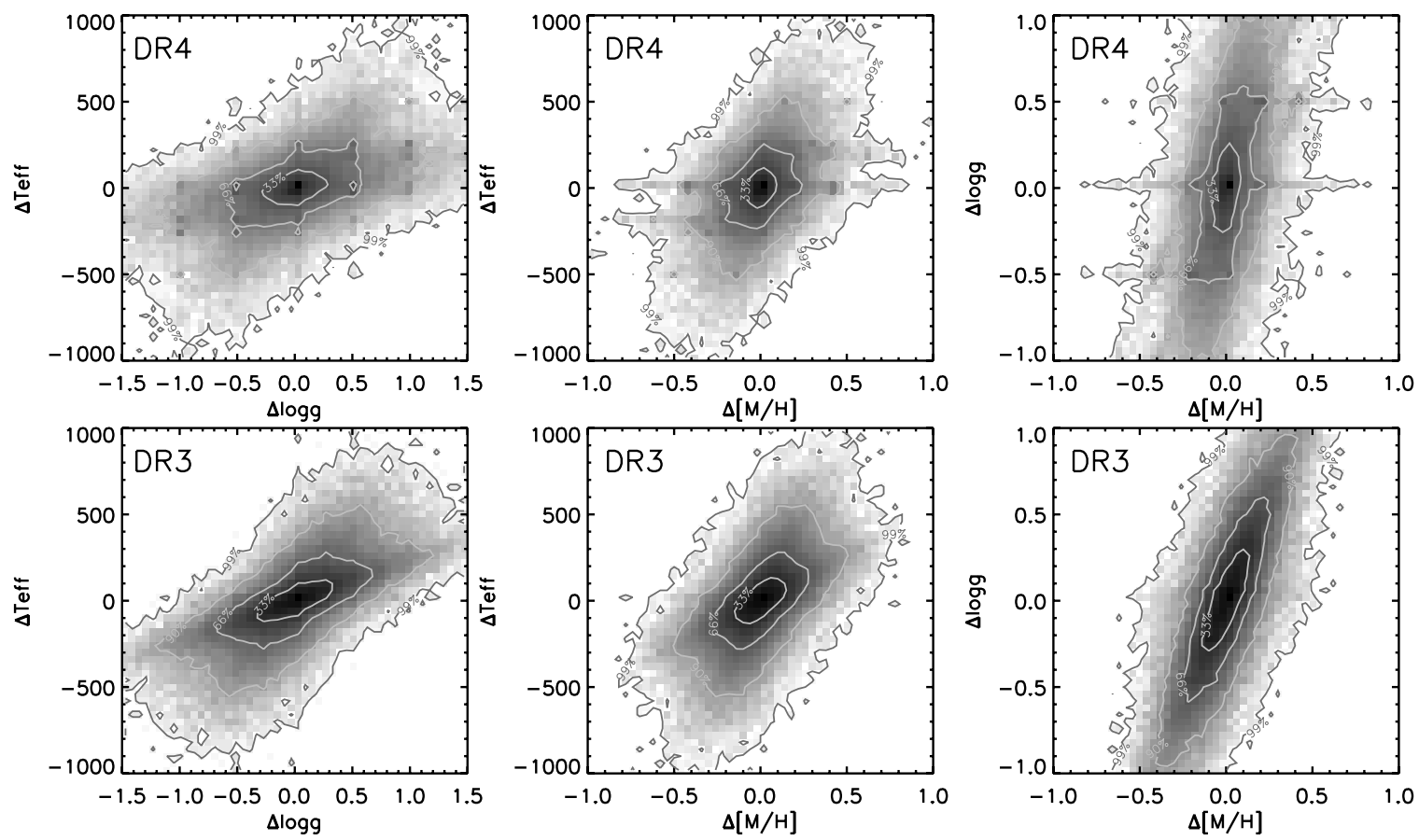

Figure 23. Correlations between the derived atmospheric parameters (top: DR4 pipeline; bottom: DR3 pipeline) for the stars that have been observed several times by RAVE. The isocontour levels contain 33\%, 66\%, 90\%, and $99 \%$ of the total considered sample. See the text for an explanation. 

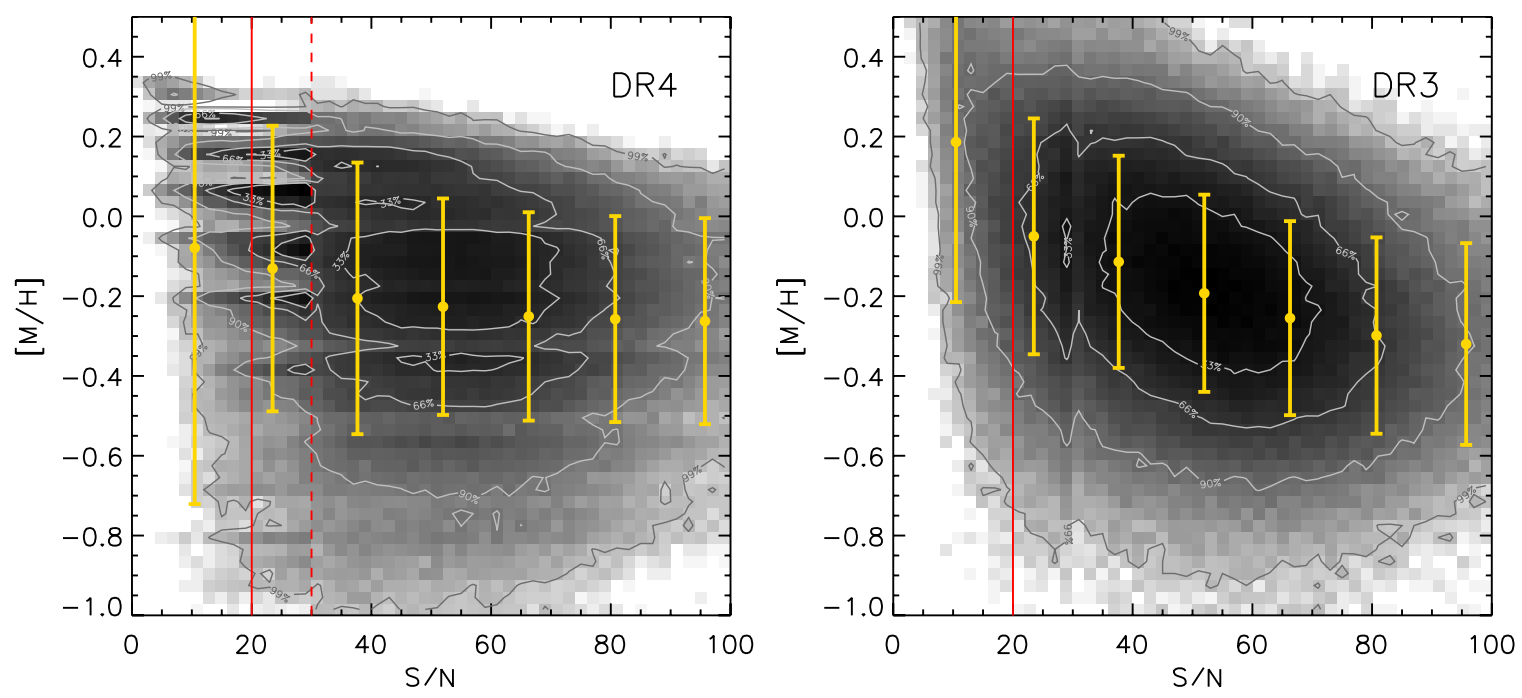

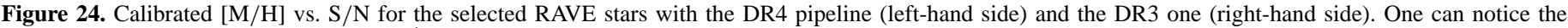

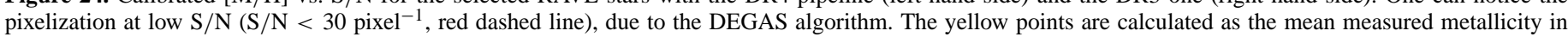

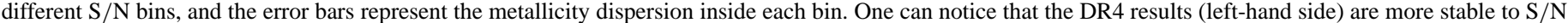

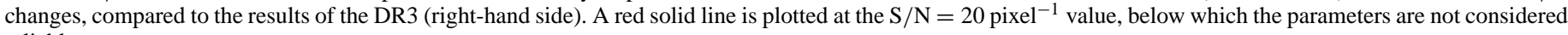
reliable.

(A color version of this figure is available in the online journal.)

Thanks to the improved line profile and the correction of the EW library for the opacity of the neighboring lines, the continuum re-normalization has improved. The new abundances are now less affected by systematic errors than the previous ones, and their correlations with $T_{\text {eff }}$ are now negligible. On the other hand, the new continuum re-normalization reveals the scarcity of information for elements with weak and few visible lines like $[\mathrm{Ca} / \mathrm{H}]$ (which has been dropped in this data release) and $[\mathrm{Ti} / \mathrm{H}]$ on dwarf stars, which turns out to be not reliable. A slight correlation between abundances and $\mathrm{S} / \mathrm{N}$ is present, as shown in Figure 26. Such correlation is negligible for giant stars, whereas for dwarf stars $[\mathrm{m} / \mathrm{H}]_{\mathrm{chem}}$ (computed with the formula given by Salaris et al. 1993, see Section 3.4 of B11) increases by $\sim 0.1$ dex from $\mathrm{S} / \mathrm{N}=80$ pixel $^{-1}$ to $\mathrm{S} / \mathrm{N}=40$ pixel $^{-1}$. The different re-normalization procedure for the two $\mathrm{S} / \mathrm{N}$ regimes generates the step in average metallicity seen at $\mathrm{S} / \mathrm{N}=40 \mathrm{pixel}^{-1}$.

The accuracy of the RAVE abundances depends on many variables, often inter-dependent in a non-linear way, which makes difficult the accuracy estimation of the individual abundances. Indeed, on one hand the abundance accuracy depends on the number of measured absorption lines. On the other hand, the number of measurable lines (i.e., strong enough to be identified in the noise) depends on $\mathrm{S} / \mathrm{N}$ and on the stellar parameters. In Figure 25 the dispersion of the residuals between measured and expected abundances (for the sample of synthetic spectra) decreases as the number of measured lines increases. This number is a useful index of goodness of the abundance accuracy, although it must be considered together with S/N. In Figure 27 we illustrate the fraction of spectra having an abundance estimation (i.e., at least one absorption line measured) for different elements, and how this fraction decreases when $\mathrm{S} / \mathrm{N}$ and/or metallicity decrease. This is a selection effect due to the measurement process, and it must be taken into account during data analysis and interpretation.

\subsubsection{Comparison between $[\mathrm{M} / \mathrm{H}]$ and $[\mathrm{m} / \mathrm{H}]_{\mathrm{chem}}$}

In order to measure the chemical abundances, the RAVE chemical pipeline uses on one hand the estimation of $T_{\text {eff }}$ and

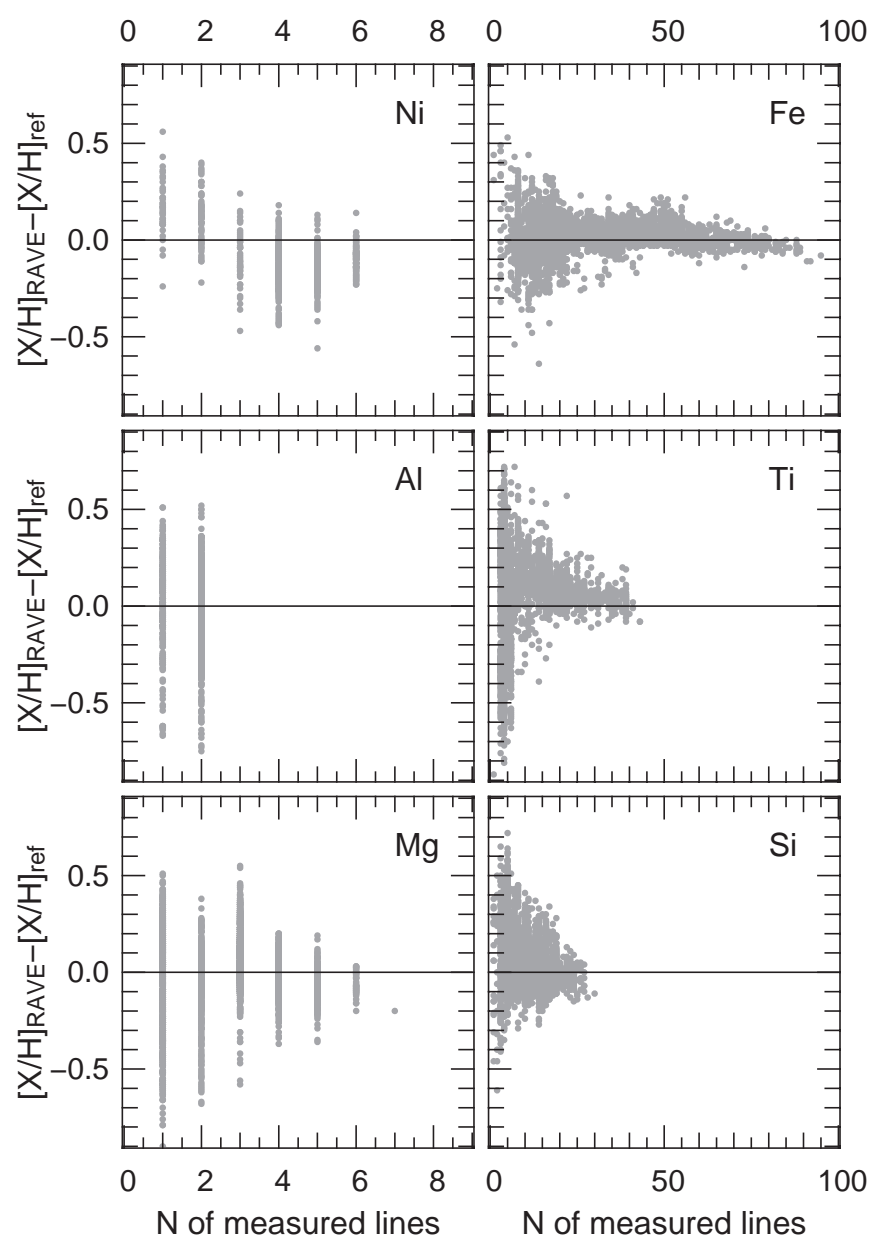

Figure 25. Residuals between measured and expected abundances ( $y$-axis) as a function of the number of measured lines ( $x$-axis) for each element in the test with synthetic spectra at $\mathrm{S} / \mathrm{N}=100,40,20 \mathrm{pixel}^{-1}$.

$\log g$ of the RAVE pipeline, and on the other hand $[\mathrm{M} / \mathrm{H}]$ is employed only as a first guess. This means that the metallicity 

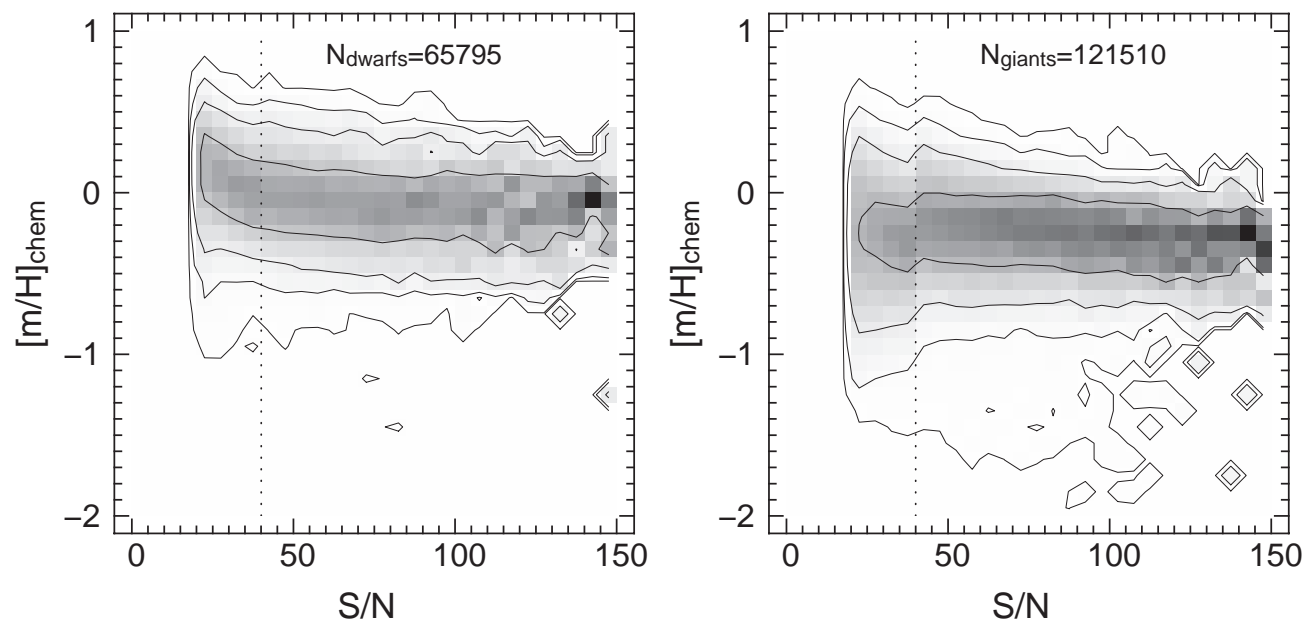

Figure 26. $[\mathrm{m} / \mathrm{H}]_{\text {chem }}$ distribution for dwarfs (left panel) and giants (right panel) as a function of $\mathrm{S} / \mathrm{N}$. In order to highlight the shape of the distribution at any $\mathrm{S} / \mathrm{N}$, the $[\mathrm{m} / \mathrm{H}]_{\mathrm{chem}}$ distribution has been normalized for every $\mathrm{S} / \mathrm{N}$ bin.

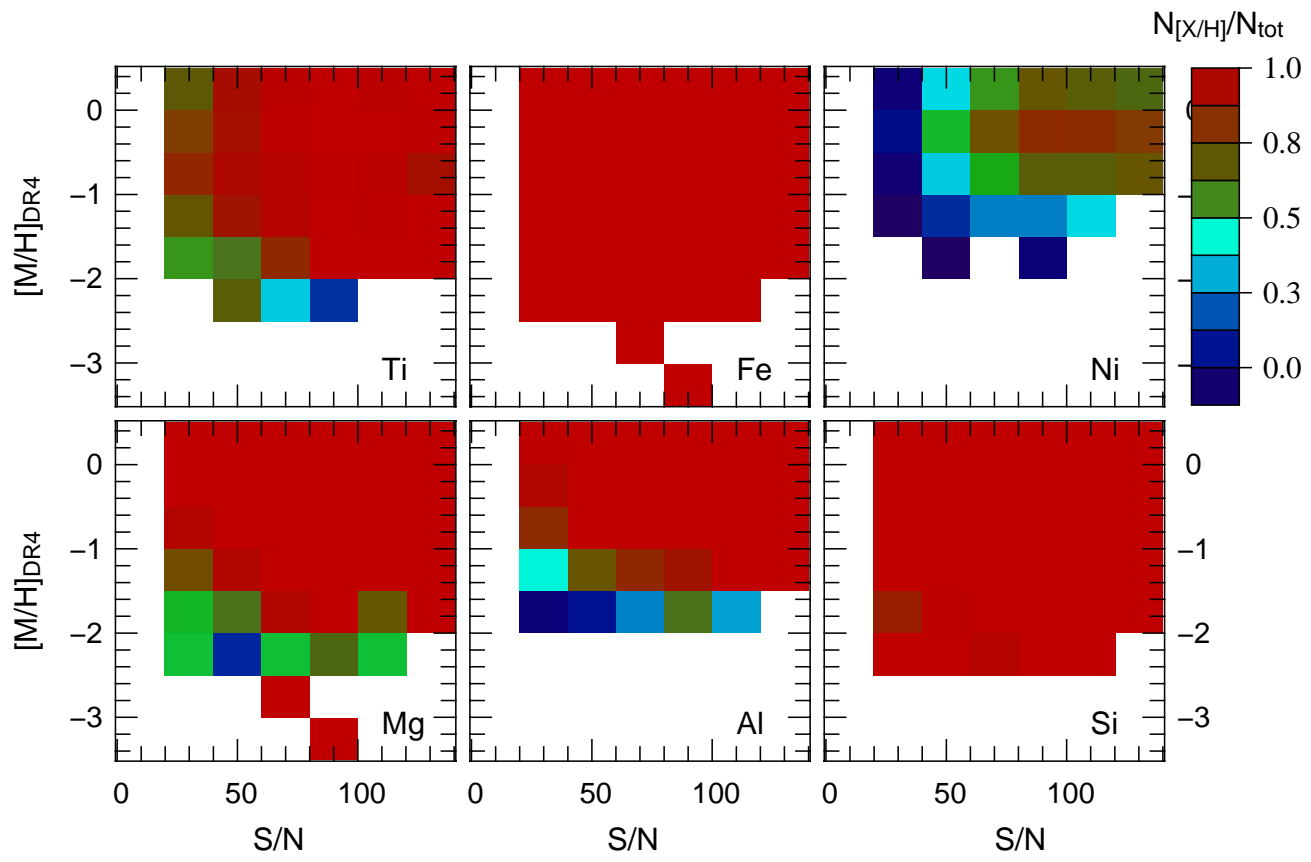

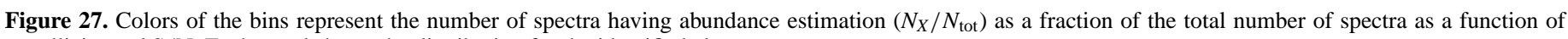
metallicity and S/N. Each panel shows the distribution for the identified element.

(A color version of this figure is available in the online journal.)

$[\mathrm{m} / \mathrm{H}]_{\text {chem }}$ (computed as explained in Section 3.4 of B11) and any elemental abundance provided by the chemical pipeline are independent of $[\mathrm{M} / \mathrm{H}]$. It is, therefore, interesting to compare $[\mathrm{M} / \mathrm{H}]$ with $[\mathrm{m} / \mathrm{H}]_{\text {chem }}$ as much as with $[\mathrm{Fe} / \mathrm{H}]$, because the latter is the element of reference used to calibrate $[\mathrm{M} / \mathrm{H}]$. In Figure 28 the residuals between $[\mathrm{M} / \mathrm{H}],[\mathrm{m} / \mathrm{H}]_{\mathrm{chem}}$, and $[\mathrm{Fe} / \mathrm{H}]$ are shown as a function of $T_{\text {eff }}, \log g$, and $[\mathrm{M} / \mathrm{H}]$ for spectra with $\mathrm{S} / \mathrm{N}>40$ pixel $^{-1}$. In general, $[\mathrm{M} / \mathrm{H}]$ appears to lie between $[\mathrm{Fe} / \mathrm{H}]$ and $[\mathrm{m} / \mathrm{H}]_{\text {chem }}$, in the order $[\mathrm{Fe} / \mathrm{H}] \leqslant[\mathrm{M} / \mathrm{H}]$ $\leqslant[\mathrm{m} / \mathrm{H}]_{\text {chem }}$. More specifically, there are some differences: for dwarf stars this difference is slightly larger $(\sim 0.1 \mathrm{dex})$ than for giants $(\leqslant 0.05 \mathrm{dex})$. This can be due to the higher number of strong and narrow absorption lines available in giants with respect to hot dwarfs, which allows better measurements over the whole $\mathrm{S} / \mathrm{N}$ range. In addition, the $[\mathrm{Fe} / \mathrm{H}]$ deviation can be due to the $\alpha$-enhanced stars, which do not follow the enhancement relation of the learning grid (see Section 3.4) and for which
$[\mathrm{Fe} / \mathrm{H}] \neq[\mathrm{M} / \mathrm{H}]$. Indeed, roughly $25 \%$ of the stars with $\mathrm{S} / \mathrm{N}>40$ pixel $^{-1}$ deviate more than $1 \sigma(0.15 \mathrm{dex})$ from the enhancements of the learning grid of Section 3.4.

\section{PROPER MOTIONS}

In DR3, the proper motions were sourced from the PPMX (Röser et al. 2008), Tycho-2 (Høg et al. 2000), SSS (Hambly et al. 2001), and UCAC2 (Zacharias et al. 2004) catalogs. As already described in DR2, the most precise available proper motion was chosen for each object. In DR4, we no longer follow this procedure but publish a set of available proper motions for each object and leave the selection to the user. The reason is the following: the proper-motion error bars published are of different origin; they may be calculated either from the scatter or from the weights of the individual positions. Hence, the proper motion with the smallest formal error is not necessarily 

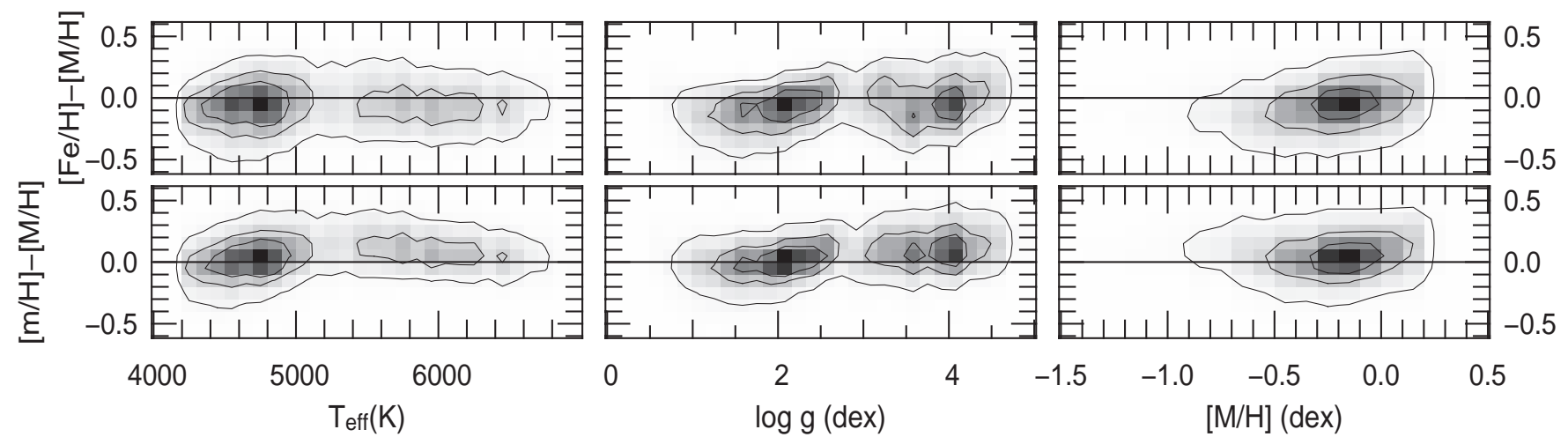

Figure 28. Residuals between the DR4 metallicity $([\mathrm{M} / \mathrm{H}])$, the chemical pipeline metallicity $([\mathrm{m} / \mathrm{H}])$, and the measured iron abundance $([\mathrm{Fe} / \mathrm{H}])$ as a function of the effective temperature, surface gravity, and metallicity. The contour levels hold 34\%, 68\%, and $95 \%$ of the points, respectively.

the most accurate. So, the DR4 catalog lists proper motions from the following sources: Tycho-2, UCAC2, UCAC3, UCAC4 (Zacharias et al. 2010, 2013), PPMX, PPMXL (Roeser et al. 2010), and SPM4 (Girard et al. 2011).

\section{RADIAL VELOCITIES}

The radial velocities for this fourth data release are based on the RAVE pipeline described in Siebert et al. (2011b); therefore, only a brief reminder of the general features is given here.

The radial velocities are obtained using a standard crosscorrelation algorithm in Fourier space on the continuum subtracted spectra. First, an estimate of the RV is obtained using a subset of 10 template spectra. This first estimate, with an accuracy better than $5 \mathrm{~km} \mathrm{~s}^{-1}$, is used to put the spectrum in the zero velocity frame. A new template is constructed using the full template database using a penalized chi-square technique described in Zwitter et al. (2008). The new template is then used to derive the final, more precise RV. The internal error is obtained as the error on the determination of the maximum of the correlation function. This part is performed using the IRAF xcsao task.

The histogram distribution of the internal RV error is presented in Figure 29 (top panel). The different histograms contain data new to each data release as indicated in the top right corner. The bottom panel of Figure 29 is the associated cumulative distribution of internal RV error. The figure shows a clear improvement of the quality of the RV from DR1 toward DR4, with a jump in quality for data new to DR4. From DR2 to DR4, ${ }^{35}$ while the mode of the distribution remains constant at $\sim 1 \mathrm{~km} \mathrm{~s}^{-1}$, the tail at larger velocity errors is consistently reduced with a leap between DR3 and DR4. Indeed, while for DR3 68\% of the data had internal errors better than $2 \mathrm{~km} \mathrm{~s}^{-1}$, for DR4 the $68 \%$ limit goes down to $1.4 \mathrm{~km} \mathrm{~s}^{-1}$. The source of this improvement is twofold. First, the DR4 data are based upon a new input catalog that uses DENIS $I$-band magnitude instead of a pseudo- $I$ magnitude constructed from Tycho- $2 B_{T}$ and $V_{T}$ photometry for the bright part of the catalog and photographic I band from the SuperCosmos Sky Survey for the faint part. This more accurate photometry allows a better splitting of the bright and faint sub-samples, which have different exposure times. Second, at the telescope the S/N is now "monitored," and fields with insufficient $\mathrm{S} / \mathrm{N}$ benefit from longer exposure times to ensure a minimal quality of the data. The combination

\footnotetext{
35 DR1 data suffer from second-order contamination, which was corrected for DR2 data; hence, the radial velocity measurement is not as precise as for subsequent releases.
}

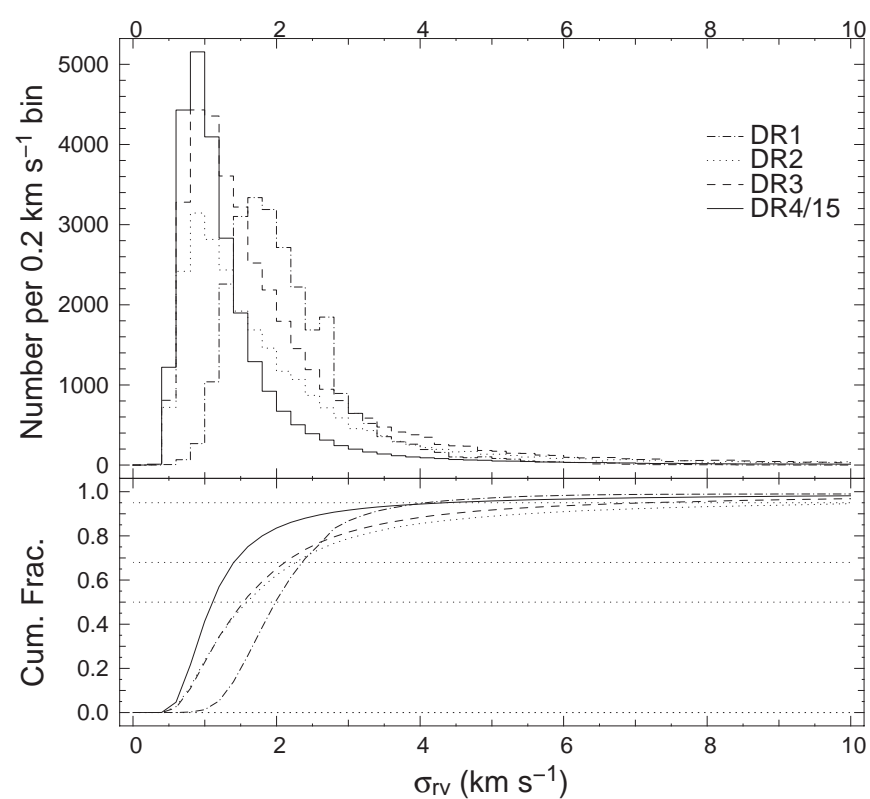

Figure 29. Top: histograms of the internal radial velocity error for data new to each data release. The bin size is $0.2 \mathrm{~km} \mathrm{~s}^{-1}$. For DR4, the number of stars per bin is divided by 15 to compensate for the increase in sample size. Bottom: cumulative distributions. The dotted lines mark 50\%, 68\%, and $95 \%$ of the samples.

of these two points allows us to considerably reduce the tail of the RV error distribution.

\subsection{Repeat Observations}

To verify the quality of the RAVE data, a fraction of the survey time is devoted to multiple observations of RAVE targets with time intervals between observations ranging from a few hours to $4 \mathrm{yr}$. In the present release, 23,288 stars belong to this program, for a total of 61,457 measurements, some stars having been observed up to 13 times. The distribution of the number of observations per star is presented in the left panel of Figure 30. The distribution of the time interval $\Delta t$ between re-observations is presented in Figure 30, right panel, where the $\Delta t$ are binned using intervals of 1 day. As seen from this figure, the distribution is not random. A quasi-logarithmic spacing is used to sample optimally the possible orbital state of the spectroscopic binaries with an enhancement of the observations at specific intervals of 1 day, 2 weeks, 1, 2, 3, and 6 months, and 1,2,3, and 4 yr.

The comparison between the RV measurements for the different observations is given in Figure 31. In the top panel, 

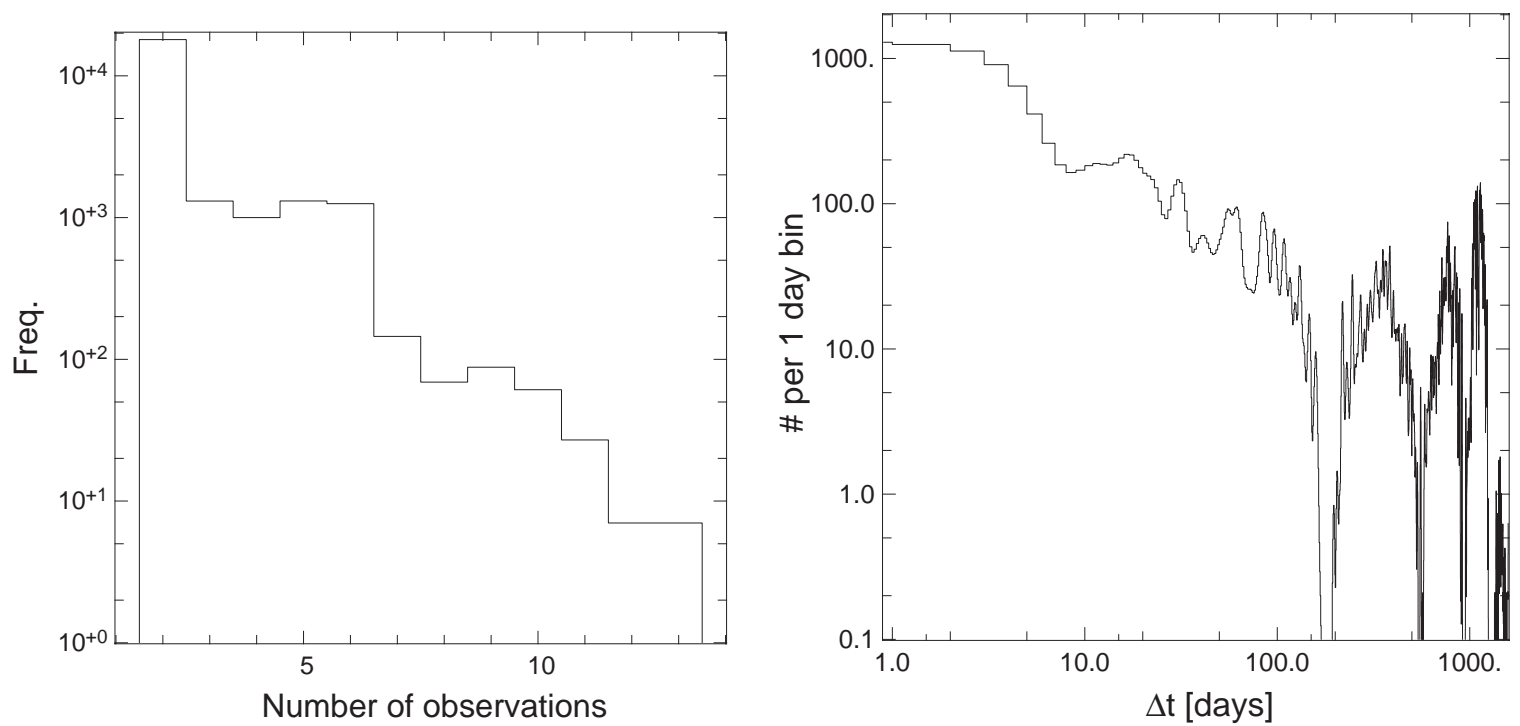

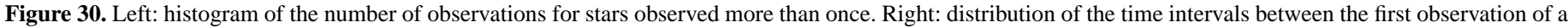
star and its re-observation.

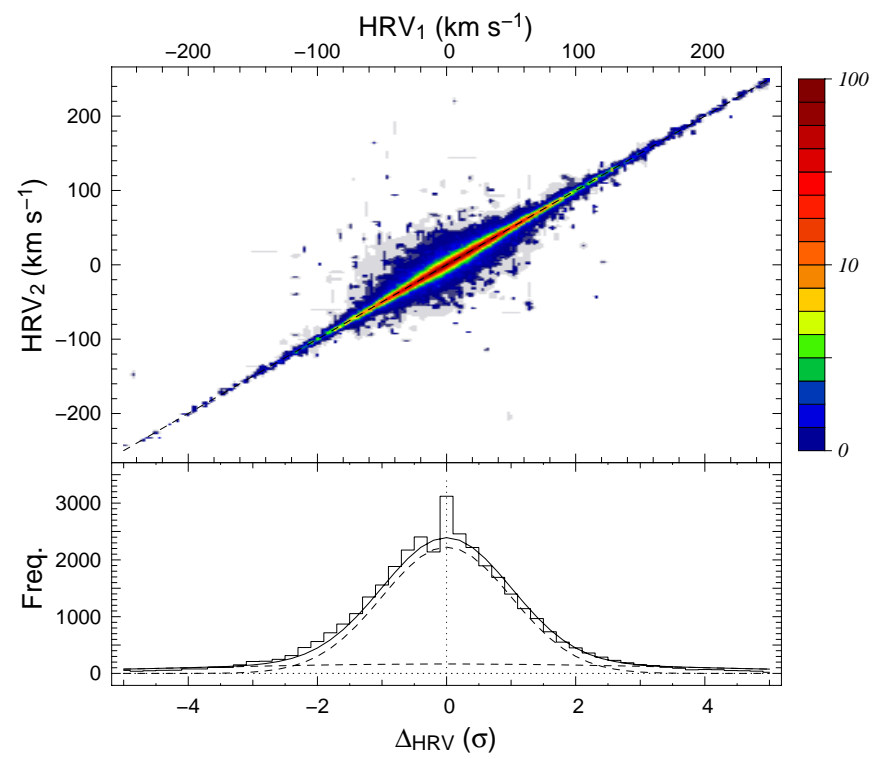

Figure 31. Top: comparison of the radial velocities measured for re-observed RAVE targets. For each star in this sample, the measurement with highest $\mathrm{S} / \mathrm{N}$ is used as the reference point on the $x$-axis $\left(\mathrm{HRV}_{1}\right)$, while other measurements are along the $y$-axis $\left(\mathrm{HRV}_{2}\right)$. Along each axis the distribution is obtained by convolving the measurement with a Gaussian function whose dispersion is the associated internal error. The color-coding follows the resulting density on a logarithmic scale. The one-to-one relation is indicated by the dashed line. Bottom: histogram of the radial velocity differences normalized to the error. The plain black line is a fit to the histogram assuming that it is composed of two Gaussian populations (see the text). From this fit, the contribution to the histogram of spectroscopic binaries and problematic spectra is $23 \%$.

(A color version of this figure is available in the online journal.)

the color coding follows the density per bin of $2 \mathrm{~km} \mathrm{~s}^{-1}$. For each star, the velocity of the observation with the highest $\mathrm{S} / \mathrm{N}$ is used as the reference velocity along the $x$-axis $\left(\mathrm{HRV}_{1}\right)$, while subsequent observations are along the $y$-axis $\left(\mathrm{HRV}_{2}\right)$. The measurements are convolved with a Gaussian function along each direction. The dispersion of the Gaussian is the internal error associated with the measurement along each axis. The general trend follows closely the one-to-one relation, showing no sign of any systematic effects.
Another way to look quantitatively at the RV difference is presented in the bottom panel of Figure 31, which shows the histogram of the RV difference normalized to the errors $\Delta_{\mathrm{HRV}}=\left(\mathrm{HRV}_{1}-\mathrm{HRV}_{2}\right) /\left(\sqrt{\sigma_{1}^{2}+\sigma_{2}^{2}}\right)$. If our measurements were perfect, this distribution should follow a Gaussian function of zero mean and unit dispersion. Binaries and problematic measurements contribute to the tail and can be assumed to follow a Gaussian function with a larger dispersion. In the case of our RAVE data, a sum of two Gaussians is used to fit the histogram, setting the dispersion of the first Gaussian function to one. Also, we remove the central bin from the fit. This central bin is mostly populated by repeat observations without delay in time. The best fit is shown as a continuous black line, the contribution of each Gaussian function being represented by a dashed line. Apart from the central bin, the fit provides an adequate representation of the observed histogram. The respective contributions of the two Gaussian functions are $77 \%$ for the standard population and $23 \%$ for the spectroscopic binaries/problematic observations. This fraction is in agreement with our finding for DR3 data $(26 \%)$.

\subsection{Zero-point Offset}

As for the DR3 and previous releases, the RV solutions are corrected for potential zero-point offsets due to change in temperature in the spectrograph room. The procedure uses the available sky lines in the RAVE spectra to construct a smooth solution of the zero-point offset across the field plate. This procedure is fully described in Siebert et al. (2011b), and the relevant measurements for each fiber are given in the catalog. To verify the validity of our velocity zero-point solution, comparison to independent measurements is made. Our comparison sample comprises data from seven different sources: the GCS (Nordström et al. 2004) data and high-resolution echelle follow-up observations of RAVE targets at the ANU $2.3 \mathrm{~m}$ telescope, Asiago Observatory, Apache Point Observatory from Ruchti et al. (2011), and Observatoire de Haute Provence using the Elodie and Sophie instruments. In total, the sample of RAVE stars with external RV measurements contains 1265 stars. Their distributions in DENIS $I$ magnitude and 2 MASS $J-H$ versus $H-K$ color-color diagram are presented in Figure 32. Stars with $I<9$ are mostly custom 

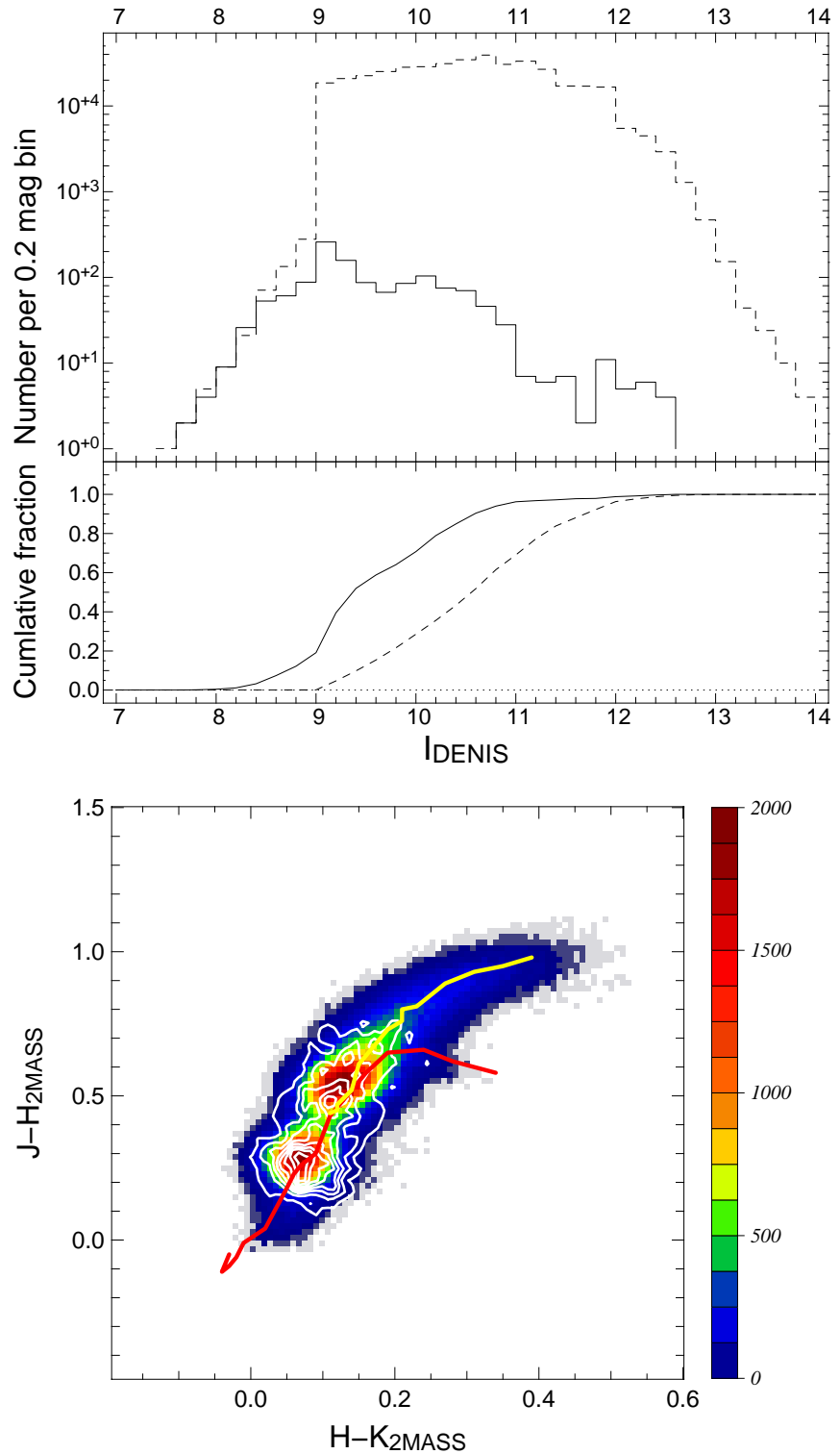

Figure 32. Top: DENIS I magnitude histogram of the sub-sample of RAVE stars with external radial velocity measurements (solid line) compared to the distribution of DENIS I magnitude for the full RAVE catalog (dashed line). Bottom: 2MASS $J-H$ vs. $H-K$ two-dimensional histogram of the RAVE DR4 catalog with a bin size of 0.02 mag on each axis. The contours show the location of the sub-sample with external HRV measurements. The thick yellow and red lines are fiducial colors from Table 2 of Wainscoat et al. (1992) for giant and dwarf stars, respectively.

(A color version of this figure is available in the online journal.)

RAVE observations of bright GCS stars. At fainter magnitudes the sample consists primarily of high-resolution re-observations of RAVE targets. In the 2MASS color-color diagram, we observe that this sample covers the two main peaks (representing dwarf and giant stars) present in the RAVE catalog. However, dwarfs are over-represented compared to the RAVE distribution due to the large number of GCS stars observed in this sample that are Hipparcos dwarfs.

The summary of the comparison to the control samples is given in Table 6 . This table reports the number of objects in each sample, the total number of observations, the mean $\Delta \mathrm{RV}=\mathrm{RV}_{\mathrm{DR} 4}-\mathrm{RV}_{\mathrm{ext}}$, and the standard deviation. $\Delta \mathrm{RV}$ is computed using an iterative $\sigma$-clipping technique to remove the contamination by spectroscopic binaries or problematic
Table 6

Summary of the Radial Velocity Comparison to External Samples

\begin{tabular}{lrrrc}
\hline \hline Sample & $N_{\text {stars }}$ & $N_{\text {obs }}$ & $\langle\Delta \mathrm{RV}\rangle$ & $\sigma_{\Delta \mathrm{RV}}\left(\sigma_{\text {clip }}, n_{\text {rej }}\right)$ \\
\hline GCS & 733 & 1024 & 0.28 & $1.72(3,120)$ \\
Chubak & 77 & 97 & -0.07 & $1.28(3,2)$ \\
Ruchti & 314 & 445 & 0.78 & $1.78(3,34)$ \\
Asiago & 25 & 47 & -0.22 & $2.95(3,0)$ \\
ANU 2.3 m & 73 & 203 & -0.60 & $2.87(3,18)$ \\
OHP Elodie & 9 & 13 & 0.29 & $0.40(2.5,3)$ \\
OHP Sophie & 34 & 43 & 0.83 & $1.56(3,4)$ \\
\hline Full sample & 1265 & 1872 & 0.20 & $1.52(3,266)$ \\
\hline
\end{tabular}

Note. The mean difference in the radial velocities, $\Delta \mathrm{RV}$, has been computed as $\Delta \mathrm{RV}=\mathrm{RV}_{\mathrm{DR} 4}-\mathrm{RV}_{\mathrm{ext}}$.

measurements. No other quality cut was applied on the samples. The clipping parameter and the number of stars rejected for each sample are given in the last column of the table.

We note that the agreement between RAVE and the external sources is better than $1 \mathrm{~km} \mathrm{~s}^{-1}$ in all the cases. Figure 33 (top panel) presents the direct comparison of the DR4 radial velocities to the external source measurements. The blue circles are the known spectroscopic binaries and show a broader distribution than the remainder of the sample. Figure 33 (bottom panel) shows the histogram of the RV difference. This distribution can be adequately reproduced by the sum of two Gaussian functions, the peak representing the single stars being of zero mean with a dispersion of $1.5 \mathrm{~km} \mathrm{~s}^{-1}$, consistent with our expectation for RAVE data.

As a final test, we check the dependence of the RV difference as a function of the Tonry-Davis correlation coefficient $(R)$ and $\mathrm{S} / \mathrm{N}$ of the RAVE data (Figure 34). Although an increase in the dispersion is observed for $\mathrm{S} / \mathrm{N}<30 \mathrm{pixel}^{-1}$, or $R$ of 40 , the absence of an apparent bias indicates that our RV measurements are reliable.

\section{STELLAR DISTANCES, AGES, AND EXTINCTIONS}

In the absence of parallaxes for the stars, the best way to obtain individual stellar distances is to project the atmospheric parameters on a set of theoretical isochrones and obtain the most likely value of the absolute magnitude of the stars. Up to now, the RAVE consortium has published a variety of studies using the distances inferred by red clump giants (e.g., Siebert et al. 2008; Veltz et al. 2008; Williams et al. 2013) and developed three different methods in order to obtain the individual stellar line-of-sight distances (Breddels et al. 2010; Zwitter et al. 2010; Burnett \& Binney 2010). Previous RAVE catalogs published the derived distances for the first two of these methods.

Figure 35 presents for different regions of the $T_{\text {eff }}-\log g$ diagram the ratio between the distances obtained using the Zwitter et al. (2010) method with the DR3 parameters (Z10-DR3 hereafter) and with the DR4 ones (Z10-DR4 hereafter). It should be noted that Z10-DR3 distances have been obtained using only internal errors for the atmospheric parameters, whereas Z10DR4 consider the quadratic sum of the external uncertainties (Table 5) and the internal ones (Table 1 or Table 2). One can see that the largest deviations in distances occur in parts of the $\mathrm{H}-\mathrm{R}$ diagram that are (and should be) scarcely populated. As an overall mean, the Z10-DR3 distances are $6 \%$ larger than the Z10-DR 4 ones, with the $1 \sigma$ dispersion being at roughly $30 \%$.

For the present DR4 catalog, we publish two sets of distances: one using the Z10 method with the DR4 parameters, as well 

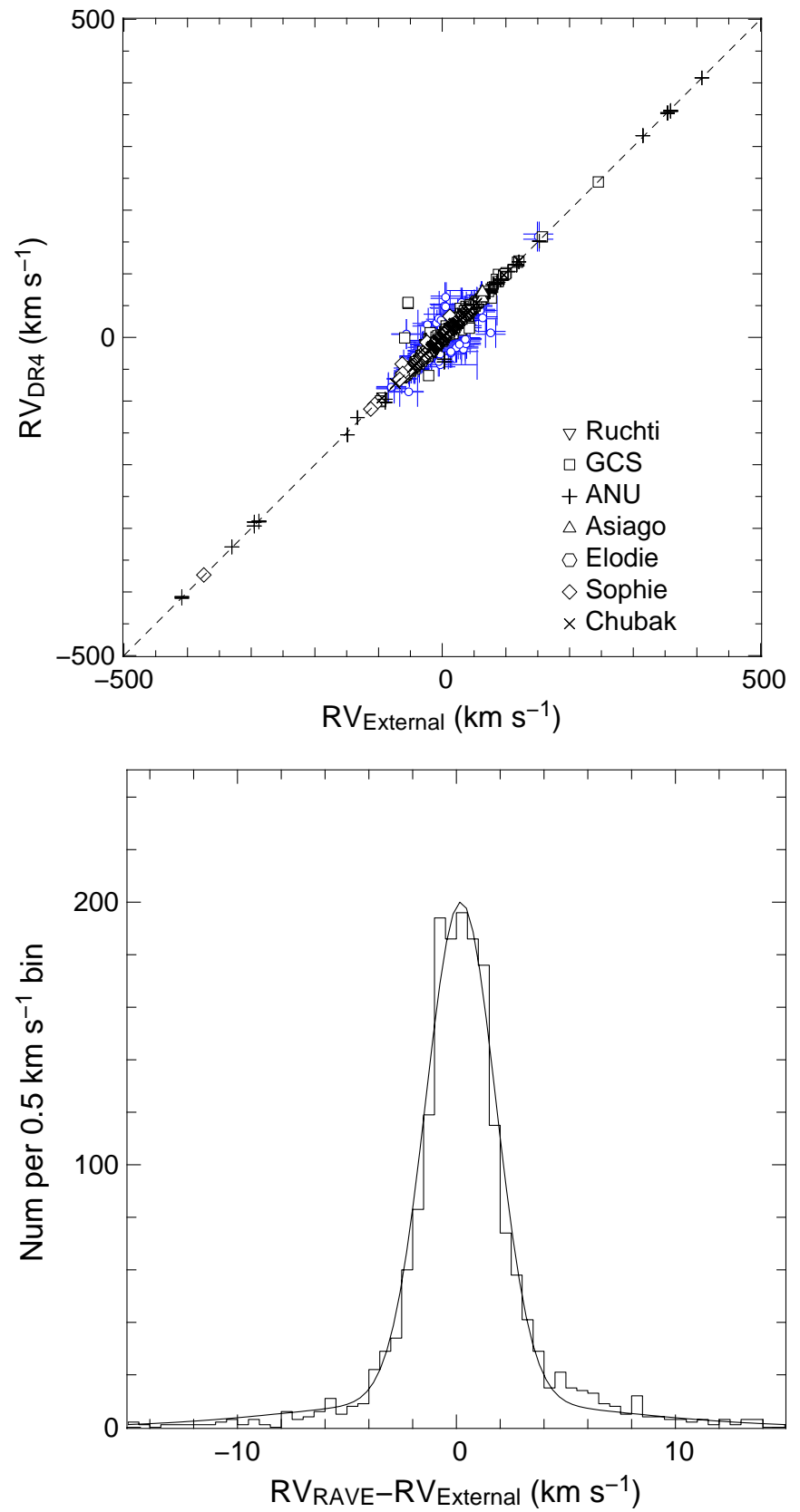

Figure 33. Top: comparison of RAVE radial velocities to external measurements. The blue circles are spectroscopic binaries detected in the high-resolution spectra. The dashed line marks the one-to-one relation. Bottom: histogram of the radial velocity difference. The histogram can be modeled using two Gaussian functions to account for normal stars and binaries/problematic spectra (black curve). The Gaussian function recovering the peak is at zero mean with a standard deviation of $1.5 \mathrm{~km} \mathrm{~s}^{-1}$. The problematic spectra/binaries contribution to this sample is $5 \%$.

(A color version of this figure is available in the online journal.)

as another set obtained using a more robust algorithm, based on the Bayesian distance-finding method of Burnett \& Binney (2010). The improved algorithm is presented in Binney et al. (2013, B13 hereafter) and now takes into account the interstellar extinction, as well as kinematic correction factors obtained by the method of Schönrich et al. (2012). The pipeline determines the probability distribution function of each star in the space of initial mass, age, metallicity, distance, extinction, etc., and from this distribution an age, a distance, and an extinction are inferred from appropriate expectation values. The most reliable
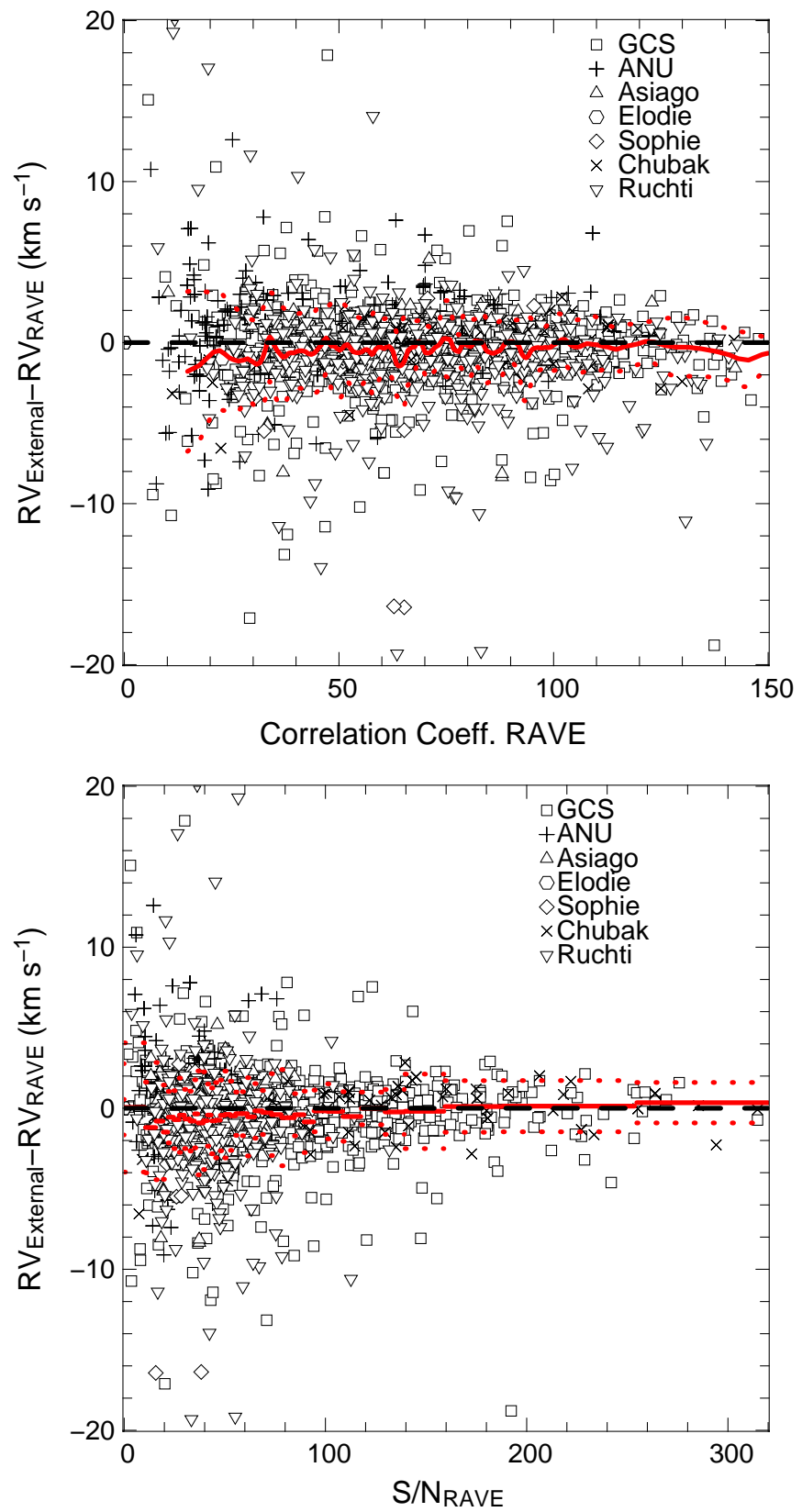

Figure 34. Radial velocity difference as a function of Tonry-Davis correlation coefficient (upper plot) and S/N (lower plot). The thick red line marks the mean relation, the red dotted line the dispersion. The symbols indicate the source of the external measurements.

(A color version of this figure is available in the online journal.)

distance indicator turns out to be not the expectation value of the distance but the inverse of the expectation value of the parallax. Here we discuss briefly the results for only the distances, but we refer the reader to B13 for a full explanation of the method, as well as a detailed analysis of the reliability of the parameters.

The B13-DR4 spectrophotometric parallaxes have been computed for the stars that had spectra with $\mathrm{S} / \mathrm{N}>10 \mathrm{pixel}^{-1}$. For the targets that were observed several times, only the spectrum with the highest $\mathrm{S} / \mathrm{N}$ has been used every time. The results have shown that the spectrophotometric parallaxes of Hipparcos stars are very satisfactory and are definitely improved by taking extinction into account. Nevertheless, an overestimation of less than $10 \%$ for the dwarfs and less than $20 \%$ for the giants is evident. The method has also been tested on the open 


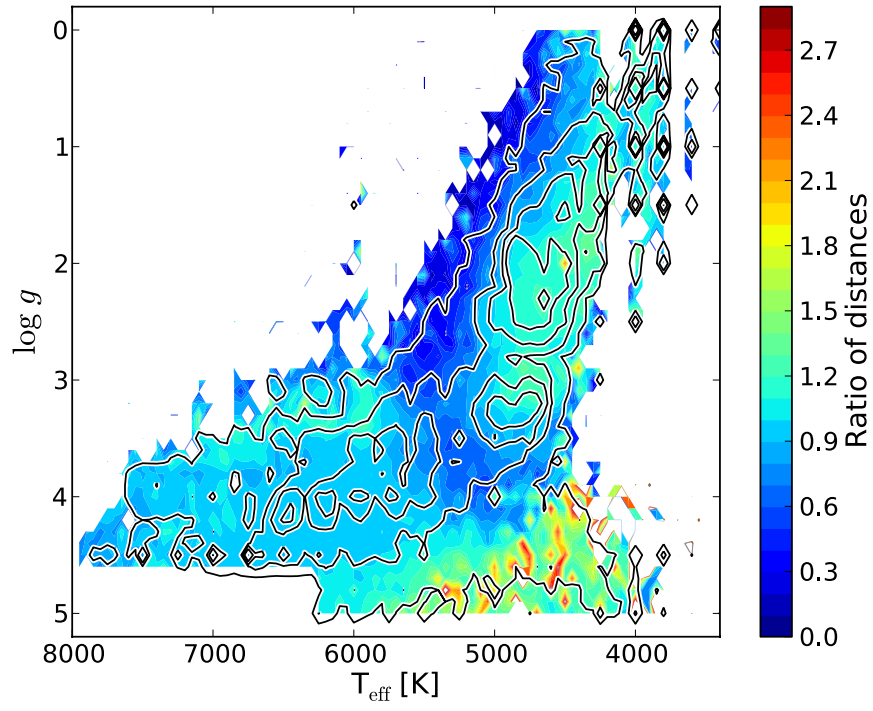

Figure 35. Comparison of the average ratio of distances in the $T_{\text {eff }}-\log g$ plot for the stellar spectra with $\mathrm{S} / \mathrm{N}>20 \mathrm{pixel}^{-1}$. The ratio is the Zwitter et al. (2010) distance based on DR3 parameters divided by the Zwitter et al. (2010) distance based on DR4 parameters. The ratio is in filled contours, bin size is $50 \mathrm{~K}$ in temperature and $0.1 \mathrm{dex}$ in gravity. Empty contours show the occurrence of $T_{\text {eff }}$ and $\log g$ (expectation values) as calculated by DR4.

(A color version of this figure is available in the online journal.)

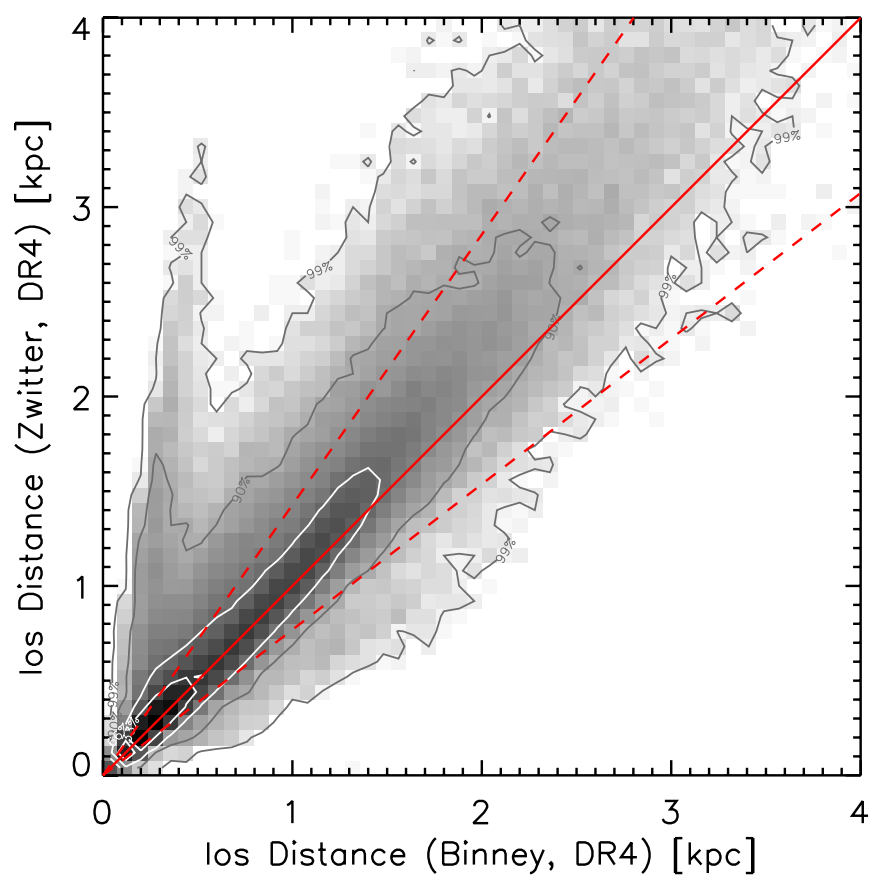

Figure 36. Comparison of the line-of-sight distance estimations obtained using the Binney et al. (2013) method ( $x$-axis) and the ones obtained using the Zwitter et al. (2010) method combined with the Padova isochrones ( $y$-axis). The red line is the 1:1 relation, and dashed lines show deviations of $30 \%$ from unity. Contour lines hold $33 \%, 66 \%, 90 \%$, and $99 \%$ of the sample.

(A color version of this figure is available in the online journal.)

cluster spectra presented in Table 4, deriving very satisfactory distances, provided that a cluster-specific age prior is used. Indeed, our data barely constrain the ages of stars, so a star's adopted age is heavily influenced by the age prior used, and the prior that is appropriate for field stars leads to excessive ages being assigned to most cluster stars, which tend to be young.

Figure 36 compares the distance estimations obtained using the Padova isochrones and the Z10-DR4 or the B13-DR4 methods. From this figure one can see that for the bulk of the targets, the distances obtained with the two methods are quite similar. We find that the median of the distribution defined by Z10/B13 is equal to 1.02 . However, for the most distant stars $(D \gtrsim 2 \mathrm{kpc}) \mathrm{Z10}$-DR4 predicts larger distances than B13-DR4. Finally, the targets for which B13-DR4 predicts $D \sim 0.5 \mathrm{kpc}$ and Z10-DR4 derives $D>1.5 \mathrm{kpc}$ correspond to the most metal-poor giant stars of the sample, for which the atmospheric parameters have also the largest errors. This disagreement is mainly due to the fact that $\mathrm{Z10}$ projects the atmospheric parameters on the isochrones, without having any a priori constraint on their expected output parameters, based on their position in the Galaxy, their RV, or proper motion. Hence, input atmospheric parameter values with large uncertainties, especially for metal-poor giant stars, naturally lead to erroneous distances and to the discrepancy shown in Figure 36.

We conclude that the B13-DR4 distances are more robust and hence should give better results if the priors are satisfied.

\section{PHOTOMETRY FROM APASS}

$B V g^{\prime} r^{\prime} i^{\prime}$ photometric data of RAVE stars have been obtained as part of the ongoing APASS survey. ${ }^{36}$ The APASS photometric survey covers the whole sky, from pole to pole, with ongoing observations from CTIO (Chile), for the southern hemisphere, and New Mexico for the northern counterpart. At both sites, a pair of twin remotely controlled, small telescopes obtain simultaneous CCD observations during dark- and gray-Moon time over five optical bands: $B, V$ (tied to the equatorial standards of Landolt 2009) and $g^{\prime}, r^{\prime}, i^{\prime}$ bands (tied to the 158 primary standards given by Smith et al. 2002, which define the Sloan photometric system). The telescopes are $20 \mathrm{~cm} f / 3.6$ astrographs feeding Apogee U16m cameras (4096 × 4096 array, $9 \mu \mathrm{m}$ pixels), which cover a field $2.9 \mathrm{deg}$ wide with a 2.6 arcsec pixel $^{-1}$ plate factor. The photometric filters are of the dielectric multi-layer type and are produced by Astrodon. Transmission curves and photometric performances of Astrodon filters used in the APASS survey are discussed and compared to more conventional types of photometric filters in Munari et al. (2012) and Munari \& Moretti (2012). On average 80 fields are observed per night at each APASS location, 20 of them being standard fields (Landolt, Sloan).

The APASS observations are obtained with fixed exposure times (different and optimized for each photometric band), set to detect $V=17$ stars at $\mathrm{S} / \mathrm{N}=5$ on a single exposure. Stars brighter than $V=10$ mag may saturate under optimal seeing conditions. At the time of writing, $90 \%$ of the whole sky has been covered, with 42 million stars measured on at least two distinct epochs. Differential photometry within a given field is accurate to better than $0.01 \mathrm{mag}$, and absolute photometry over the whole sky is currently accurate to better than 0.025 mag (closely similar to 2MASS accuracy). APASS astrometric positions are also highly accurate. Comparison with the positions given in the Carlsberg Meridian Catalog for the 118,940 RAVE stars in common shows a distribution peaked at a separation of 0.105 arcsec, with the median value at 0.177 arcsec. Although APASS DR7 is publicly available, its values are not published in RAVE DR4, because future APASS DR will provide better accuracy and coverage of RAVE DR4. Clearly APASS photometry will significantly enhance analysis of RAVE data. We recommend users to adopt APASS photometry as it becomes available.

\footnotetext{
36 http://www.aavso.org./apass
} 
Table 7

Catalog Description

\begin{tabular}{|c|c|c|c|c|c|}
\hline Col & Format & Units & NULL & Label & Explanations \\
\hline 1 & $\operatorname{char}(32)$ & $\cdots$ & $\mathrm{N}$ & RAVE_OBS_ID & Target designation \\
\hline 2 & $\operatorname{char}(18)$ & $\ldots$ & $\mathrm{N}$ & RAVEID & RAVE target designation \\
\hline 3 & double & deg & $\mathrm{N}$ & RAdeg & Right ascension \\
\hline 4 & double & deg & $\mathrm{N}$ & DEdeg & Declination \\
\hline 5 & double & deg & $\mathrm{N}$ & Glon & Galactic longitude \\
\hline 6 & double & $\operatorname{deg}$ & $\mathrm{N}$ & Glat & Galactic latitude \\
\hline 7 & float & $\mathrm{km} \mathrm{s}^{-1}$ & $\mathrm{~N}$ & HRV & Heliocentric radial velocity \\
\hline 8 & float & $\mathrm{km} \mathrm{s}^{-1}$ & $\mathrm{~N}$ & eHRV & HRV error \\
\hline 9 & float & $(\mathrm{R}+)$ & $\mathrm{N}$ & CorrelationCoeff & Tonry and Davis $R$ correlation coefficient \\
\hline 10 & float & $\ldots$ & $\mathrm{N}$ & PeakHeight & Height of correlation peak \\
\hline 11 & float & $\ldots$ & $\mathrm{N}$ & PeakWidth & Width of correlation peak \\
\hline 12 & float & $\mathrm{km} \mathrm{s}^{-1}$ & $\mathrm{Y}$ & SkyRV & Measured HRV of sky \\
\hline 13 & float & $\mathrm{km} \mathrm{s}^{-1}$ & $\mathrm{Y}$ & eSkyRV & Error measured HRV of sky \\
\hline 14 & float & $(\mathrm{R}+)$ & $\mathrm{Y}$ & SkyCorrelationCoeff & Tonry and Davis $R$ sky correlation coefficient \\
\hline 15 & float & $\mathrm{km} \mathrm{s}^{-1}$ & $\mathrm{Y}$ & CorrectionRV & Zero-point correction applied radial velocity \\
\hline 16 & $\operatorname{char}(5)$ & $\ldots$ & $\mathrm{Y}$ & ZeroPointFLAG & Quality flag for zero-point correction (Note 3 ) \\
\hline 17 & float & $\ldots$ & $\mathrm{Y}$ & STN_SPARV & $\mathrm{S} / \mathrm{N}$ value (preflux calibration) (Note 1$)$ \\
\hline 18 & $\operatorname{char}(16)$ & $\ldots$ & $\mathrm{Y}$ & ID_TYCHO2 & TYCHO2 target designation \\
\hline 19 & float & $\operatorname{arcsec}$ & $\mathrm{Y}$ & Dist_TYCHO2 & Center distance to target catalog \\
\hline 20 & $\operatorname{char}(2)$ & $\ldots$ & $\mathrm{Y}$ & XidQualityFLAG_TYCHO2 & Crossmatch quality flag (Note 4) \\
\hline 21 & float & mas $\mathrm{yr}^{-1}$ & $\mathrm{Y}$ & pmRA_TYCHO2 & Proper-motion RA from TYCHO2 \\
\hline 22 & float & $\operatorname{mas} \mathrm{yr}^{-1}$ & $\mathrm{Y}$ & epmRA_TYCHO2 & Error proper-motion RA from TYCHO2 \\
\hline 23 & float & $\operatorname{mas} \mathrm{yr}^{-1}$ & $\mathrm{Y}$ & pmDE_TYCHO2 & Proper-motion DE from TYCHO2 \\
\hline 24 & float & $\operatorname{mas} \mathrm{yr}^{-1}$ & $\mathrm{Y}$ & epmDE_TYCHO2 & Error proper-motion DE from $\mathrm{TYCHO} 2$ \\
\hline 25 & $\operatorname{char}(16)$ & $\ldots$ & $\mathrm{Y}$ & ID_UCAC2 & UCAC2 target designation \\
\hline 26 & float & $\operatorname{arcsec}$ & $\mathrm{Y}$ & Dist_UCAC2 & Center distance to target catalog \\
\hline 27 & $\operatorname{char}(2)$ & $\ldots$ & $\mathrm{Y}$ & XidQualityFLAG_UCAC2 & Crossmatch quality flag (Note 4 ) \\
\hline 28 & float & mas $\mathrm{yr}^{-1}$ & $\mathrm{Y}$ & pmRA_UCAC2 & Proper-motion RA from UCAC2 \\
\hline 29 & float & $\operatorname{mas} \mathrm{yr}^{-1}$ & $\mathrm{Y}$ & epmRA_UCAC2 & Error proper-motion RA from UCAC2 \\
\hline 30 & float & $\operatorname{mas} \mathrm{yr}^{-1}$ & $\mathrm{Y}$ & pmDE_UCAC2 & Proper-motion DE from UCAC2 \\
\hline 31 & float & $\operatorname{mas} \mathrm{yr}^{-1}$ & $\mathrm{Y}$ & epmDE_UCAC2 & Error proper-motion DE from UCAC2 \\
\hline 32 & $\operatorname{char}(16)$ & $\ldots$ & $\mathrm{Y}$ & ID_UCAC3 & UCAC3 target designation \\
\hline 33 & float & $\operatorname{arcsec}$ & $\mathrm{Y}$ & Dist_UCAC3 & Center distance to target catalog \\
\hline 34 & $\operatorname{char}(2)$ & $\ldots$ & $\mathrm{Y}$ & XidQualityFLAG_UCAC3 & Crossmatch quality flag (Note 4) \\
\hline 35 & float & $\operatorname{mas} \mathrm{yr}^{-1}$ & $\mathrm{Y}$ & pmRA_UCAC3 & Proper-motion RA from UCAC3 \\
\hline 36 & float & mas $\mathrm{yr}^{-1}$ & $\mathrm{Y}$ & epmRA_UCAC3 & Error proper-motion RA from UCAC3 \\
\hline 37 & float & mas $\mathrm{yr}^{-1}$ & $\mathrm{Y}$ & pmDE_UCAC3 & Proper-motion DE from UCAC3 \\
\hline 38 & float & mas $\mathrm{yr}^{-1}$ & $\mathrm{Y}$ & epmDE_UCAC3 & error Proper-motion DE from UCAC3 \\
\hline 39 & $\operatorname{char}(16)$ & $\ldots$ & $\mathrm{Y}$ & ID_UCAC4 & UCAC4 target designation \\
\hline 40 & float & $\operatorname{arcsec}$ & $\mathrm{Y}$ & Dist_UCAC4 & Center distance to target catalog \\
\hline 41 & $\operatorname{char}(2)$ & $\ldots$ & $\mathrm{Y}$ & XidQualityFLAG_UCAC4 & Crossmatch quality flag (Note 4) \\
\hline 42 & float & mas $\mathrm{yr}^{-1}$ & $\mathrm{Y}$ & pmRA_UCAC4 & Proper-motion RA from UCAC4 \\
\hline 43 & float & $\operatorname{mas} \mathrm{yr}^{-1}$ & $\mathrm{Y}$ & epmRA_UCAC4 & Error proper-motion RA from UCAC4 \\
\hline 44 & float & $\operatorname{mas} \mathrm{yr}^{-1}$ & $\mathrm{Y}$ & pmDE_UCAC4 & Proper-motion DE from UCAC4 \\
\hline 45 & float & mas $\mathrm{yr}^{-1}$ & $\mathrm{Y}$ & epmDE_UCAC4 & Error proper-motion DE from UCAC4 \\
\hline 46 & $\operatorname{char}(16)$ & $\ldots$ & $\mathrm{Y}$ & ID_PPMXL & PPMXL target designation \\
\hline 47 & float & $\operatorname{arcsec}$ & $\mathrm{Y}$ & Dist_PPMXL & Center distance to target catalog \\
\hline 48 & $\operatorname{char}(2)$ & $\ldots$ & $\mathrm{Y}$ & XidQualityFLAG_PPMXL & Crossmatch quality flag (Note 4) \\
\hline 49 & float & $\operatorname{mas} \mathrm{yr}^{-1}$ & $\mathrm{Y}$ & pmRA_PPMXL & Proper-motion RA from PPMXL \\
\hline 50 & float & mas $\mathrm{yr}^{-1}$ & $\mathrm{Y}$ & epmRA_PPMXL & Error proper-motion RA from PPMXL \\
\hline 51 & float & mas $\mathrm{yr}^{-1}$ & $\mathrm{Y}$ & pmDE_PPMXL & Proper-motion DE from PPMXL \\
\hline 52 & float & $\operatorname{mas} \mathrm{yr}^{-1}$ & $\mathrm{Y}$ & epmDE_PPMXL & Error proper-motion DE from PPMXL \\
\hline 53 & $\operatorname{char}(10)$ & $\ldots$ & $\mathrm{N}$ & Obsdate & Observation date yyyymmdd \\
\hline 54 & $\operatorname{char}(14)$ & $\ldots$ & $\mathrm{N}$ & FieldName & Name of RAVE field (RA/DE) \\
\hline 55 & int & $\ldots$ & $\mathrm{N}$ & PlateNumber & Number of field plate [1..3] \\
\hline 56 & int & $\ldots$ & $\mathrm{N}$ & FiberNumber & Number of optical fiber $[1,150]$ \\
\hline 57 & float & $\mathrm{K}$ & $\mathrm{Y}$ & Teff_K & Effective temperature (Note 1) \\
\hline 58 & float & $\mathrm{K}$ & $\mathrm{Y}$ & eTeff_K & Error effective temperature (Note 1 ) \\
\hline 59 & float & dex & $\mathrm{Y}$ & $\operatorname{logg\_ } K$ & Log gravity (Note 1$)$ \\
\hline 60 & float & dex & $\mathrm{Y}$ & elogg_K & Error log gravity (Note 1) \\
\hline 61 & float & $\operatorname{dex}$ & $\mathrm{Y}$ & Met_K & Metallicity $[\mathrm{m} / \mathrm{H}]$ (Note 1$)$ \\
\hline 62 & float & dex & $\mathrm{Y}$ & Met_N_K & Metallicity $[\mathrm{m} / \mathrm{H}]($ Note 1$)$ \\
\hline 63 & float & dex & $\mathrm{Y}$ & eMet_K & Error/metallicity $[\mathrm{m} / \mathrm{H}]($ Note 1$)$ \\
\hline 64 & float & $\ldots$ & $\mathrm{Y}$ & SNR_K & $\mathrm{S} / \mathrm{N}$ value (Note 1$)$ \\
\hline 65 & float & $\ldots$ & $\mathrm{Y}$ & Algo_Conv_K & Quality flag for stellar parameter pipeline $[0 \ldots 4]$ (Note 1, Note 5) \\
\hline 66 & float & dex & $\mathrm{Y}$ & $\mathrm{Al}$ & Abundance of $\mathrm{Al}[\mathrm{Al} / \mathrm{H}]$ \\
\hline
\end{tabular}


Table 7

(Continued)

\begin{tabular}{|c|c|c|c|c|c|}
\hline $\mathrm{Col}$ & Format & Units & NULL & Label & Explanations \\
\hline 67 & int & $\ldots$ & $\mathrm{Y}$ & Al_N & Number of used spectral lines for calculation of abundance \\
\hline 68 & float & dex & $\mathrm{Y}$ & $\mathrm{Si}$ & Abundance of $\mathrm{Si}[\mathrm{Si} / \mathrm{H}]$ \\
\hline 69 & int & $\ldots$ & $\mathrm{Y}$ & $\mathrm{Si} \_\mathrm{N}$ & Number of used spectral lines for calculation of abundance \\
\hline 70 & float & dex & $\mathrm{Y}$ & $\mathrm{Fe}$ & Abundance of $\mathrm{Fe}[\mathrm{Fe} / \mathrm{H}]$ \\
\hline 71 & int & $\ldots$ & $\mathrm{Y}$ & $\mathrm{Fe} \_\mathrm{N}$ & Number of used spectral lines for calculation of abundance \\
\hline 72 & float & dex & $\mathrm{Y}$ & $\mathrm{Ti}$ & Abundance of $\mathrm{Ti}[\mathrm{Ti} / \mathrm{H}]$ \\
\hline 73 & int & $\ldots$ & $\mathrm{Y}$ & Ti_N & Number of used spectral lines for calculation of abundance \\
\hline 74 & float & dex & $\mathrm{Y}$ & $\mathrm{Ni}$ & Abundance of $\mathrm{Ni}[\mathrm{Ni} / \mathrm{H}]$ \\
\hline 75 & int & $\ldots$ & $\mathrm{Y}$ & $\mathrm{Ni} N \mathrm{~N}$ & Number of used spectral lines for calculation of abundance \\
\hline 76 & float & dex & $\mathrm{Y}$ & $\mathrm{Mg}$ & Abundance of $\mathrm{Mg}[\mathrm{Mg} / \mathrm{H}]$ \\
\hline 77 & int & $\ldots$ & $\mathrm{Y}$ & Mg_N & Number of used spectral lines for calculation of abundance \\
\hline 78 & float & $\ldots$ & $\mathrm{Y}$ & CHISQ_c & $\chi^{2}$ of the chemical pipeline (Note 1$)$ \\
\hline 79 & float & $\mathrm{K}$ & $\mathrm{Y}$ & Teff_SPARV & Effective temperature (Note 1) \\
\hline 80 & float & dex & $\mathrm{Y}$ & logg_SPARV & Log gravity (Note 1$)$ \\
\hline 81 & float & dex & Y & alpha_SPARV & Metallicity (Note 1) \\
\hline 82 & $\operatorname{char}(16)$ & $\ldots$ & Y & ID_2MASS & 2MASS target designation \\
\hline 83 & float & $\operatorname{arcsec}$ & $\mathrm{Y}$ & Dist_2MASS & Center distance to target catalog \\
\hline 84 & $\operatorname{char}(2)$ & $\ldots$ & $\mathrm{Y}$ & XidQualityFLAG_2MASS & Crossmatch quality flag (Note 4 ) \\
\hline 85 & double & mag & Y & Jmag_2MASS & $J$ magnitude \\
\hline 86 & double & mag & $\mathrm{Y}$ & eJmag_2MASS & Error $J$ magnitude \\
\hline 87 & double & mag & $\mathrm{Y}$ & Hmag_2MASS & $H$ magnitude \\
\hline 88 & double & mag & $\mathrm{Y}$ & eHmag_2MASS & Error $H$ magnitude \\
\hline 89 & double & mag & $\mathrm{Y}$ & Kmag_2MASS & $K$ magnitude \\
\hline 90 & double & mag & $\mathrm{Y}$ & eKmag_2MASS & Error $K$ magnitude \\
\hline 91 & char(16) & $\ldots$ & $\mathrm{Y}$ & ID_DENIS & DENIS target designation \\
\hline 92 & double & $\operatorname{arcsec}$ & $\mathrm{Y}$ & Dist_DENIS & Center distance to target catalog \\
\hline 93 & $\operatorname{char}(2)$ & $\ldots$ & $\mathrm{Y}$ & XidQualityFLAG_DENIS & Crossmatch quality flag (Note 4 ) \\
\hline 94 & double & mag & Y & Imag_DENIS & $I$ magnitude \\
\hline 95 & double & mag & $\mathrm{Y}$ & eImag_DENIS & Error $I$ magnitude \\
\hline 96 & double & mag & $\mathrm{Y}$ & Jmag_DENIS & $J$ magnitude \\
\hline 97 & double & mag & Y & eJmag_DENIS & Error $J$ magnitude \\
\hline 98 & double & mag & $\mathrm{Y}$ & Kmag_DENIS & $K$ magnitude \\
\hline 99 & double & mag & $\mathrm{Y}$ & eKmag_DENIS & Error $K$ magnitude \\
\hline 100 & $\operatorname{char}(16)$ & $\ldots$ & $\mathrm{Y}$ & ID_USNOB1 & USNOB1 target designation \\
\hline 101 & double & $\operatorname{arcsec}$ & $\mathrm{Y}$ & Dist_USNOB1 & Center distance to target catalog \\
\hline 102 & $\operatorname{char}(2)$ & $\ldots$ & $\mathrm{Y}$ & XidQualityFLAG_USNOB1 & Crossmatch quality flag (Note 4) \\
\hline 103 & double & mag & $\mathrm{Y}$ & B1mag_USNOB1 & B1 magnitude \\
\hline 104 & double & mag & $\mathrm{Y}$ & R1mag_USNOB1 & R1 magnitude \\
\hline 105 & double & mag & $\mathrm{Y}$ & B2mag_USNOB1 & B2 magnitude \\
\hline 106 & double & mag & $\mathrm{Y}$ & R2mag_USNOB1 & R2 magnitude \\
\hline 107 & double & mag & $\mathrm{Y}$ & Imag_USNOB1 & $I$ magnitude \\
\hline 108 & float & mas & $\mathrm{Y}$ & parallax & Parallax (Note 4) \\
\hline 109 & float & mas & $\mathrm{Y}$ & e_parallax & Error parallax (Note 4) \\
\hline 110 & float & $\mathrm{kpc}$ & $\mathrm{Y}$ & dist & Distance (Note 4) \\
\hline 111 & float & $\mathrm{kpc}$ & $\mathrm{Y}$ & e_dist & Error distance (Note 4) \\
\hline 112 & float & $\ldots$ & $\mathrm{Y}$ & DistanceModulus_Binney & Distance modulus (Note 4) \\
\hline 113 & float & $\ldots$ & $\mathrm{Y}$ & eDistanceModulus_Binney & Distance modulus (Note 4) \\
\hline 114 & float & $\ldots$ & $\mathrm{Y}$ & $\mathrm{Av}$ & Extinction (Note 4) \\
\hline 115 & float & $\ldots$ & Y & e_Av & Error extinction (Note 4) \\
\hline 116 & float & $\ldots$ & $\mathrm{Y}$ & age & Age (Note 4) \\
\hline 117 & float & $\ldots$ & $\mathrm{Y}$ & e_age & Error age (Note 4) \\
\hline 118 & float & MSun & $\mathrm{Y}$ & mass & Mass (Note 4) \\
\hline 119 & float & MSun & $\mathrm{Y}$ & e_mass & Error mass (Note 4) \\
\hline 120 & $\operatorname{char}(2)$ & $\ldots$ & $\mathrm{Y}$ & $\mathrm{c} 1$ & n.th minimum distance (Note 6) \\
\hline 121 & $\operatorname{char}(2)$ & $\ldots$ & $\mathrm{Y}$ & c2 & n.th minimum distance (Note 6) \\
\hline 122 & $\operatorname{char}(2)$ & $\ldots$ & $\mathrm{Y}$ & c3 & n.th minimum distance (Note 6) \\
\hline 123 & $\operatorname{char}(2)$ & $\ldots$ & $\mathrm{Y}$ & c4 & n.th minimum distance (Note 6 ) \\
\hline 124 & $\operatorname{char}(2)$ & $\ldots$ & $\mathrm{Y}$ & $\mathrm{c5}$ & n.th minimum distance (Note 6 ) \\
\hline 125 & $\operatorname{char}(2)$ & $\ldots$ & $\mathrm{Y}$ & c6 & n.th minimum distance (Note 6) \\
\hline 126 & $\operatorname{char}(2)$ & $\ldots$ & $\mathrm{Y}$ & c7 & n.th minimum distance (Note 6) \\
\hline 127 & $\operatorname{char}(2)$ & $\ldots$ & $\mathrm{Y}$ & c8 & n.th minimum distance (Note 6) \\
\hline 128 & $\operatorname{char}(2)$ & $\ldots$ & $\mathrm{Y}$ & c9 & n.th minimum distance (Note 6 ) \\
\hline 129 & $\operatorname{char}(2)$ & $\ldots$ & $\mathrm{Y}$ & $\mathrm{c} 10$ & n.th minimum distance (Note 6) \\
\hline 130 & $\operatorname{char}(2)$ & $\ldots$ & $\mathrm{Y}$ & c11 & n.th minimum distance (Note 6) \\
\hline 131 & $\operatorname{char}(2)$ & $\ldots$ & $\mathrm{Y}$ & c12 & n.th minimum distance (Note 6) \\
\hline
\end{tabular}


Table 7

(Continued)

\begin{tabular}{|c|c|c|c|c|c|}
\hline Col & Format & Units & NULL & Label & Explanations \\
\hline 132 & $\operatorname{char}(2)$ & $\ldots$ & $\mathrm{Y}$ & $\mathrm{c} 13$ & n.th minimum distance (Note 6) \\
\hline 133 & $\operatorname{char}(2)$ & $\ldots$ & $\mathrm{Y}$ & c14 & n.th minimum distance (Note 6) \\
\hline 134 & $\operatorname{char}(2)$ & $\ldots$ & $\mathrm{Y}$ & $\mathrm{c} 15$ & n.th minimum distance (Note 6) \\
\hline 135 & $\operatorname{char}(2)$ & $\ldots$ & $\mathrm{Y}$ & $\mathrm{c} 16$ & n.th minimum distance (Note 6) \\
\hline 136 & $\operatorname{char}(2)$ & $\ldots$ & $\mathrm{Y}$ & $\mathrm{c} 17$ & n.th minimum distance (Note 6) \\
\hline 137 & $\operatorname{char}(2)$ & $\ldots$ & $\mathrm{Y}$ & $\mathrm{c} 18$ & n.th minimum distance (Note 6) \\
\hline 138 & $\operatorname{char}(2)$ & $\ldots$ & $\mathrm{Y}$ & c19 & n.th minimum distance (Note 6) \\
\hline 139 & $\operatorname{char}(2)$ & $\ldots$ & $\mathrm{Y}$ & $\mathrm{c} 20$ & n.th minimum distance (Note 6) \\
\hline
\end{tabular}

Notes. (1) Originating from:_K indicates values from stellar parameter pipeline,_N_K indicates a calibrated value, _c indicates values from chemical pipeline, _SPARV indicates values of radial velocity pipeline (used in DR3 also). (2) Cross-identification flag as follows: $A=1$ association within 2 arcsec; $B=2$ associations within $2 \operatorname{arcsec} ; C=$ more than 2 associations within $2 \operatorname{arcsec} ; D=$ nearest neighbor more than $2 \operatorname{arcsec}$ away; $X=$ no association found (within 10 arcsec limit). (3) Flag value of the form FGSH, F being for the entire plate, $\mathrm{G}$ for the 50 fiber group to which the fiber belongs. $\mathrm{S}$ flags the zero-point correction used: $\mathrm{C}$ for cubic and $\mathrm{S}$ for a constant shift. If $\mathrm{H}$ is set to * the fiber is close to a 15 fiber gap. For $\mathrm{F}$ and $\mathrm{G}$ the values can be $\mathrm{A}, \mathrm{B}, \mathrm{C}, \mathrm{D}$, or $\mathrm{E}: A=$ dispersion around correction lower than $1 \mathrm{~km} \mathrm{~s}^{-1} ; B=$ dispersion between 1 and $2 \mathrm{~km} \mathrm{~s}^{-1} ; C=$ dispersion between 2 and $3 \mathrm{~km} \mathrm{~s}{ }^{-1} ; D=$ dispersion larger than $3 \mathrm{~km} \mathrm{~s}^{-1} ; E=$ less than 15 fibers available for the fit. (4) See Binney et al. (2013). (5) Flag of stellar parameter pipeline: $0=$ pipeline converged; $1=$ no convergence; 2 = MATISSE oscillates between two values and the mean has been performed; $3=$ results of MATISSE at the boundaries or outside the grid and the DEGAS value has been adopted; $4=$ the metal-poor giants with $\mathrm{S} / \mathrm{N}<20$ have been re-run by DEGAS with a scale factor (i.e., internal parameter of DEGAS) of 0.40. (6) Morphological Flag n.th minimum distance to base spectrum given by one of the types $a, b, c, d, e, g, h, n, o, p, t, u, w$ (see Matijevič et al. 2012).

\section{CONCLUSIONS}

The fourth public data release of the RAVE survey includes the stellar atmospheric parameters of 482,430 spectra obtained from 2004 April to 2012 December. Compared to the previous catalog of DR3, a new input catalog, based on DENIS DR3 and 2MASS, is used to select the observed targets. The new input catalog has the major new feature of extending to lower Galactic latitudes and providing more accurate astrometry, leading to fiber placement better matching stellar positions on the sky, which results in higher $\mathrm{S} / \mathrm{N}$ spectra. In addition, the parameters have been revisited, thanks to a new pipeline, presented in Kordopatis et al. (2011a), and 809 reference spectra that allowed us to validate the effective temperatures and surface gravities and calibrate the metallicities. The RAVE stellar atmospheric parameters that are obtained with the new pipeline are free of any obvious systematics (no correlations between the derived parameters or as a function of $\mathrm{S} / \mathrm{N}$ ), in particular for the overall metallicities of the stars. The spectra with the lowest $\mathrm{S} / \mathrm{N}$ have a distribution function of atmospheric parameter values that shows a well-understood pattern of discretization effects, but it has been shown that this discretization does not alter the accuracy of the derived parameters. We show that the MDFs of the observed stars shift toward lower metallicity values for the lower surface gravity bins, at the same time as the RV dispersion increases. This is in agreement with a change in the mixture of the probed Galactic populations as a function of the probed volume, passing from an old thin disk dominated population to the presence of more halo stars for the larger volume probed by the more luminous giant stars in low surface gravity bins. That is, at face value the distribution functions for derived stellar parameters are consistent with plausible astrophysical expectations.

In addition to the atmospheric parameters obtained with the new pipeline, those obtained with the DR3 pipeline are also published since they have been used in published analyses. However, they are of lower reliability than our DR4 data set, so situations demanding their re-analysis should be rather rare.

The abundances of six individual elements, namely, aluminum, silicon, titanium, iron, magnesium, and nickel, are published, using an improved version of the Boeche et al. (2011) chemical pipeline. The reliability of these elemental abundances varies according to the effective temperature, surface gravity, and metallicity of the star, the $\mathrm{S} / \mathrm{N}$ of the spectrum, and of course the element itself.

The catalog also includes the line-of-sight distances computed using the methods presented in Binney et al. (2013) and Zwitter et al. (2010), as well as the ages and the interstellar extinctions that are a sub-product of the Binney et al. (2013) pipeline. Radial velocities, photometric information, proper motions, and stellar binarity flags complete the DR4 catalog entries.

We are most grateful to our referee, for his detailed and very relevant comments, which improved the quality of the presentation of the paper.

Funding for RAVE has been provided by the Australian Astronomical Observatory; the Leibniz-Institut für Astrophysik Potsdam (AIP); the Australian National University; the Australian Research Council; the French National Research Agency; the German Research Foundation (SPP 1177 and SFB 881); the European Research Council (ERC-StG 240271 Galactica); the Istituto Nazionale di Astrofisica at Padova; the Johns Hopkins University; the National Science Foundation of the USA (AST0908326); the W. M. Keck Foundation; the Macquarie University; the Netherlands Research School for Astronomy; the Natural Sciences and Engineering Research Council of Canada; the Slovenian Research Agency; the Swiss National Science Foundation; the Science \& Technology Facilities Council of the UK; Opticon; Strasbourg Observatory; and the Universities of Groningen, Heidelberg, and Sydney. The RAVE Web site is at http://www.rave-survey.org.

\section{APPENDIX}

Table 7 describes the contents of individual columns of the Fourth Data Release catalog. The catalog is accessible online at http://www.rave-survey.org and via the CDS VizieR service.

\section{REFERENCES}

Abadi, M. G., Navarro, J. F., Steinmetz, M., \& Eke, V. R. 2003, ApJ, 591, 499 Allende Prieto, C., Barklem, P. S., Lambert, D. L., \& Cunha, K. 2004, A\&A, 420,183 
Alvarez, R., \& Plez, B. 1998, A\&A, 330, 1109

Antoja, T., Helmi, A., Bienayme, O., et al. 2012, MNRAS, 426, L1

Asplund, M., Grevesse, N., \& Sauval, A. J. 2005, in ASP Conf. Ser. 336, Cosmic Abundances as Records of Stellar Evolution and Nucleosynthesis, ed. T. G. Barnes, III \& F. N. Bash (San Francisco, CA: ASP), 25

Bijaoui, A., Recio-Blanco, A., de Laverny, P., \& Ordenovic, C. 2012, StMet, 9 , 55

Binney, J., Burnett, B., Kordopatis, G., et al. 2013, arXiv:1309.4270

Boeche, C., Siebert, A., Williams, M., et al. 2011, AJ, 142, 193

Brault, J., \& Neckel, H. 1987, Spectral Atlas of Solar Absolute DiskAveraged and Disk-Center Intensity from 3290 to $12510 \AA$, Available from ftp.hs.uni-hamburg.de/pub/outgoing/FTS-Atlas/

Breddels, M. A., Smith, M. C., Helmi, A., et al. 2010, A\&A, 511, A90

Bruce, S. F., Higinbotham, J., Marshall, I., \& Beswick, P. H. 2000, JMagR, 142,57

Burnett, B., \& Binney, J. 2010, MNRAS, 407, 339

Burnett, B., Binney, J., Sharma, S., et al. 2011, A\&A, 532, A113

Carretta, E., Bragaglia, A., \& Cacciari, C. 2004, ApJL, 610, L25

Casagrande, L., Ramírez, I., Meléndez, J., Bessell, M., \& Asplund, M. 2010, A\&A, 512, A54

Casagrande, L., Schönrich, R., Asplund, M., et al. 2011, A\&A, 530, A138

Cayrel, R., Depagne, E., Spite, M., et al. 2004, A\&A, 416, 1117

Chubak, C., Marcy, G., Fischer, D. A., et al. 2012, arXiv:1207.6212

DENIS Consortium. 2003, yCat, 1, 2002

DENIS Consortium. 2005, yCat, 1, 2002

Freeman, K., \& Bland-Hawthorn, J. 2002, ARA\&A, 40, 487

Fuhrmann, K. 1998a, A\&A, 338, 161

Fuhrmann, K. 1998b, A\&A, 330, 626

Fuhrmann, K. 2004, AN, 325, 3

Fuhrmann, K. 2008, MNRAS, 384, 173

Fulbright, J. P., Wyse, R. F. G., Ruchti, G. R., et al. 2010, ApJL, 724, L104

Girard, T. M., van Altena, W. F., Zacharias, N., et al. 2011, AJ, 142, 15

Gratton, R., Sneden, C., \& Carretta, E. 2004, ARA\&A, 42, 385

Gratton, R. G., Bonifacio, P., Bragaglia, A., et al. 2001, A\&A, 369, 87

Gratton, R. G., Carretta, E., \& Castelli, F. 1996, A\&A, 314, 191

Gratton, R. G., Carretta, E., Claudi, R., Lucatello, S., \& Barbieri, M. 2003, A\&A, 404, 187

Grevesse, N. 2008, CoAst, 157, 156

Grevesse, N., \& Sauval, A. J. 1998, SSRv, 85, 161

Gustafsson, B., Edvardsson, B., Eriksson, K., et al. 2008, A\&A, 486, 951

Hambly, N. C., MacGillivray, H. T., Read, M. A., et al. 2001, MNRAS, 326,1279

Hekker, S., \& Meléndez, J. 2007, A\&A, 475, 1003

Helmi, A., White, S. D. M., de Zeeuw, P. T., \& Zhao, H. 1999, Natur, 402, 53

Hinkle, K., Wallace, L., Livingston, W., et al. 2003, in The Future of Cool-Star Astrophysics: 12th Cambridge Workshop on Cool Stars, Stellar Systems, and the Sun (2001 July 30-August 3), Vol. 12, ed. A. Brown, G. M. Harper, $\&$ T. R. Ayres (Boulder, CO: Univ. Colorado), 851

Høg, E., Fabricius, C., Makarov, V. V., et al. 2000, A\&A, 355, L27

Kielkopf, J. F. 1973, JOSA, 63, 987

Kirby, E. N., Guhathakurta, P., Bolte, M., Sneden, C., \& Geha, M. C. 2009, ApJ, 705,328

Koch, A., \& McWilliam, A. 2011, AJ, 142, 63

Kordopatis, G., Recio-Blanco, A., de Laverny, P., et al. 2011a, A\&A, 535, A106

Kordopatis, G., Recio-Blanco, A., de Laverny, P., et al. 2011b, A\&A, 535, A107

Landolt, A. U. 2009, AJ, 137, 4186

Luck, R. E., \& Heiter, U. 2006, AJ, 131, 3069
Luck, R. E., \& Heiter, U. 2007, AJ, 133, 2464

Matijevič, G., Zwitter, T., Bienaymé, O., et al. 2011, AJ, 141, 200

Matijevič, G., Zwitter, T., Bienaymé, O., et al. 2012, ApJS, 200, 14

Matijevič, G., Zwitter, T., Munari, U., et al. 2010, AJ, 140, 184

McWilliam, A. 1990, ApJS, 74, 1075

Mishenina, T. V., Bienaymé, O., Gorbaneva, T. I., et al. 2006, A\&A, 456,1109

Mishenina, T. V., \& Kovtyukh, V. V. 2001, A\&A, 370, 951

Mishenina, T. V., Soubiran, C., Bienaymé, O., et al. 2008, A\&A, 489, 923

Mishenina, T. V., Soubiran, C., Kovtyukh, V. V., \& Korotin, S. A. 2004, A\&A, 418,551

Munari, U., Bacci, S., Baldinelli, L., et al. 2012, BaltA, 21, 13

Munari, U., \& Moretti, S. 2012, BaltA, 21, 22

Munari, U., Sordo, R., Castelli, F., \& Zwitter, T. 2005, A\&A, 442, 1127

Nordström, B., Mayor, M., Andersen, J., et al. 2004, A\&A, 418, 989

Pace, G., Pasquini, L., \& François, P. 2008, A\&A, 489, 403

Pancino, E., Carrera, R., Rossetti, E., \& Gallart, C. 2010, A\&A, 511, A56

Pasetto, S., Grebel, E. K., Zwitter, T., et al. 2012a, A\&A, 547, A70

Pasetto, S., Grebel, E. K., Zwitter, T., et al. 2012b, A\&A, 547, A71

Pasquini, L., Randich, S., Zoccali, M., et al. 2004, A\&A, 424, 951

Ramírez, I., Allende Prieto, C., \& Lambert, D. L. 2007, A\&A, 465, 271

Ramírez, S. V., \& Cohen, J. G. 2003, AJ, 125, 224

Recio-Blanco, A., Bijaoui, A., \& de Laverny, P. 2006, MNRAS, 370, 141

Robin, A. C., Reylé, C., Derrière, S., \& Picaud, S. 2003, A\&A, 409, 523

Roeser, S., Demleitner, M., \& Schilbach, E. 2010, AJ, 139, 2440

Röser, S., Schilbach, E., Schwan, H., et al. 2008, A\&A, 488, 401

Ruchti, G. R., Fulbright, J. P., Wyse, R. F. G., et al. 2011, ApJ, 737, 9

Salaris, M., Chieffi, A., \& Straniero, O. 1993, ApJ, 414, 580

Sales, L. V., Helmi, A., Abadi, M. G., et al. 2009, MNRAS, 400, L61

Schönrich, R., Binney, J., \& Asplund, M. 2012, MNRAS, 420, 1281

Seabroke, G. M., Gilmore, G., Siebert, A., et al. 2008, MNRAS, 384, 11

Sellwood, J. A., \& Binney, J. J. 2002, MNRAS, 336, 785

Siebert, A., Bienaymé, O., Binney, J., et al. 2008, MNRAS, 391, 793

Siebert, A., Famaey, B., Binney, J., et al. 2012, MNRAS, 425, 2335

Siebert, A., Famaey, B., Minchev, I., et al. 2011a, MNRAS, 412, 2026

Siebert, A., Williams, M. E. K., Siviero, A., et al. 2011b, AJ, 141, 187

Smith, J. A., Tucker, D. L., Kent, S., et al. 2002, AJ, 123, 2121

Sneden, C. A. 1973, PhD thesis, Univ. Texas Austin

Soubiran, C., \& Girard, P. 2005, A\&A, 438, 139

Soubiran, C., Le Campion, J.-F., Cayrel de Strobel, G., \& Caillo, A. 2010, A\&A, 515, A111

Steinmetz, M., Zwitter, T., Siebert, A., et al. 2006, AJ, 132, 1645

Valdes, F., Gupta, R., Rose, J. A., Singh, H. P., \& Bell, D. J. 2004, ApJS, 152,251

Valenti, J. A., \& Fischer, D. A. 2005, ApJS, 159, 141

van der Kruit, P. C., \& Freeman, K. C. 2011, ARA\&A, 49, 301

Veltz, L., Bienaymé, O., Freeman, K. C., et al. 2008, A\&A, 480, 753

Wainscoat, R. J., Cohen, M., Volk, K., Walker, H. J., \& Schwartz, D. E. 1992, ApJS, 83, 111

Williams, M. E. K., Steinmetz, M., Binney, J., et al. 2013, arXiv:1302.2468

Williams, M. E. K., Steinmetz, M., Sharma, S., et al. 2011, ApJ, 728, 102

Wilson, M. L., Helmi, A., Morrison, H. L., et al. 2011, MNRAS, 413, 2235

Zacharias, N., Finch, C., Girard, T., et al. 2010, AJ, 139, 2184

Zacharias, N., Finch, C. T., Girard, T. M., et al. 2013, AJ, 145, 44

Zacharias, N., Urban, S. E., Zacharias, M. I., et al. 2004, AJ, 127, 3043

Zwitter, T., Matijevič, G., Breddels, M. A., et al. 2010, A\&A, 522, A54

Zwitter, T., Siebert, A., Munari, U., et al. 2008, AJ, 136, 421 